\title{
The Reactivity of Thymine and Thymidine 5,6-Epoxides with Organometallic Reagents - A Route to Thymidine (6-4) Photoproduct Analogues
}

Pauli Wrigstedt, ${ }^{[a]}$ Jari Kavakka, ${ }^{[b]}$ Sami Heikkinen, ${ }^{[b]}$ Martin Nieger ${ }^{[a]}$ Minna Räisänen, ${ }^{[a]}$ and Timo Repo ${ }^{*[a]}$

${ }^{[a]}$ Department of Chemistry, Laboratory of Inorganic Chemistry, University of Helsinki, P. O. Box 55, FIN00014, Finland.

${ }^{[b]}$ Department of Chemistry, Laboratory of Organic Chemistry, University of Helsinki, P. O. Box 55, FIN00014, Finland.

Supporting Information

Table of Contents

Copy of NMR spectra of compounds 1-30 S2-S38

Crystallographic data of compounds $3, \mathbf{9 , 1 8}$ and 25 S39-S42

Cartesian coordinates for all optimized structures. S43-S55

Total energies of compounds $\mathbf{1}$ and 3-11. . 556

Spatial distance differences of $\mathrm{H}-6$ and $\mathrm{CH}_{3}-9$ between diastereoisomers of $\mathbf{1}$ and 3-11 S56

Putative reaction mechanism for the formation 2-oxazolidone derivative $\mathbf{3 0}$ S57 

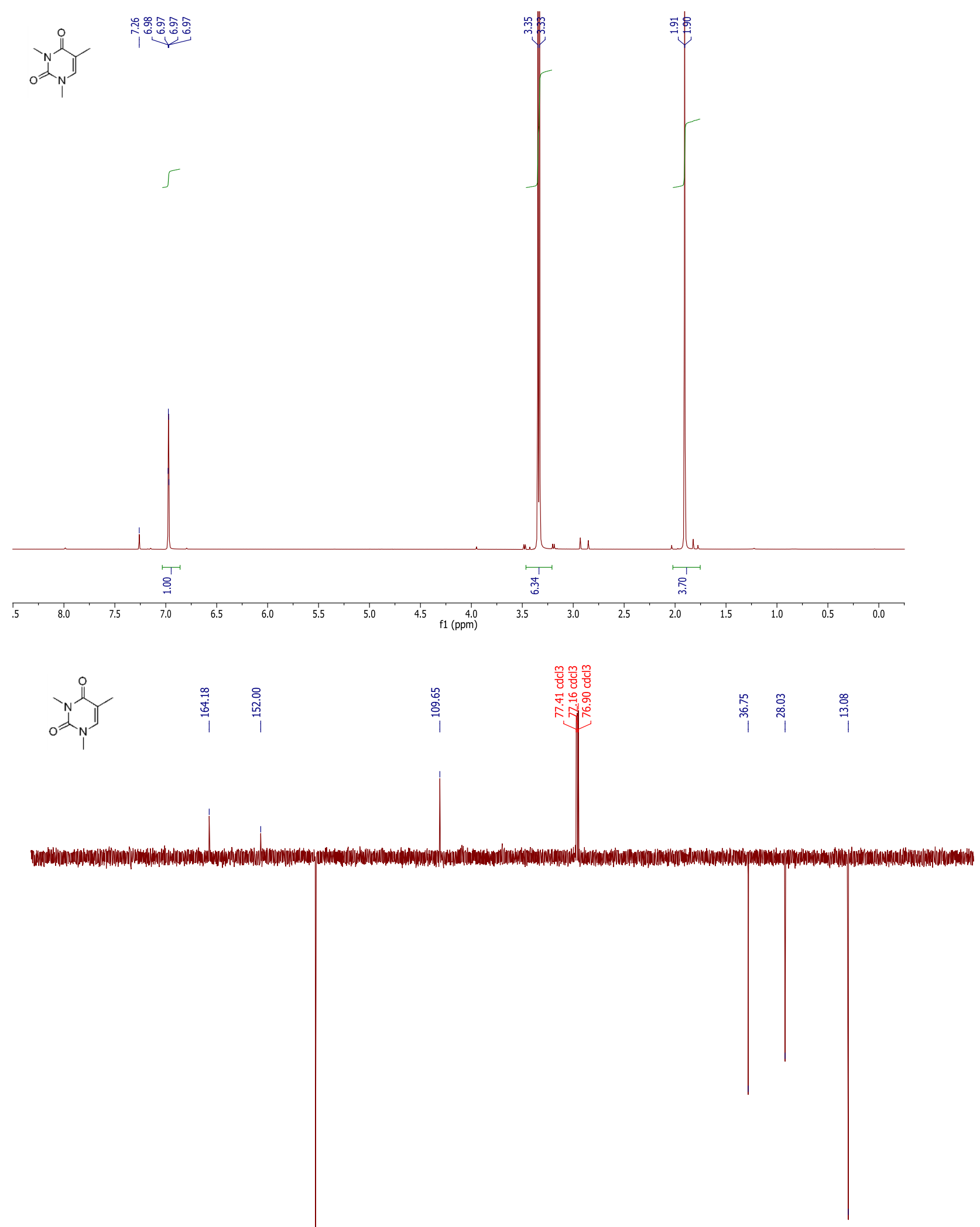

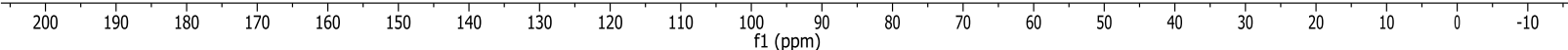



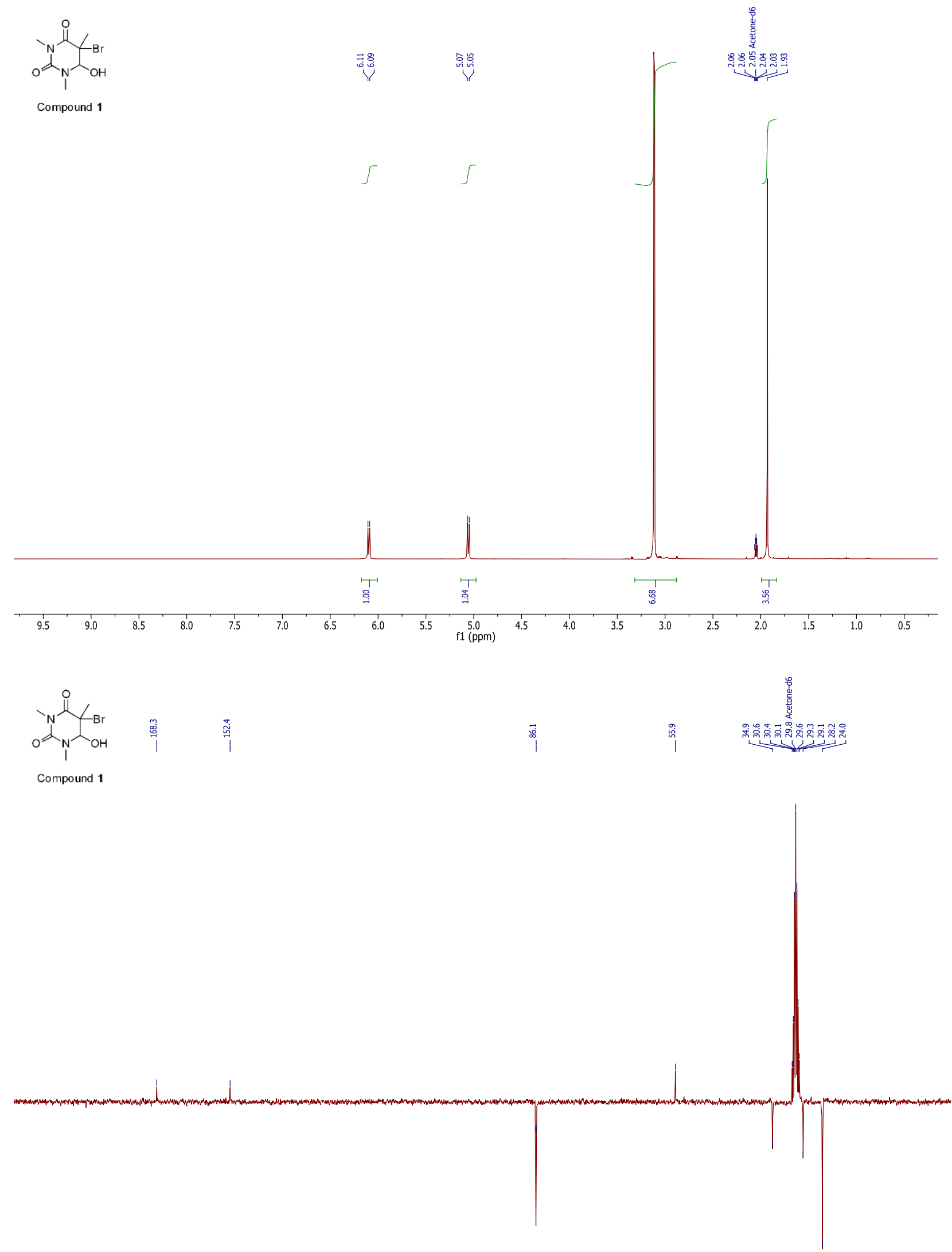

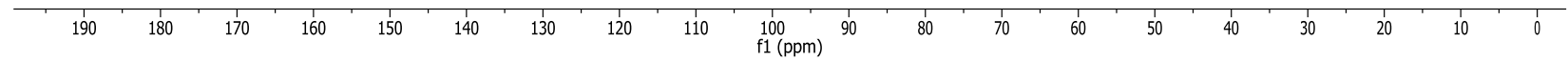




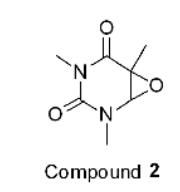

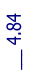

望

TEA

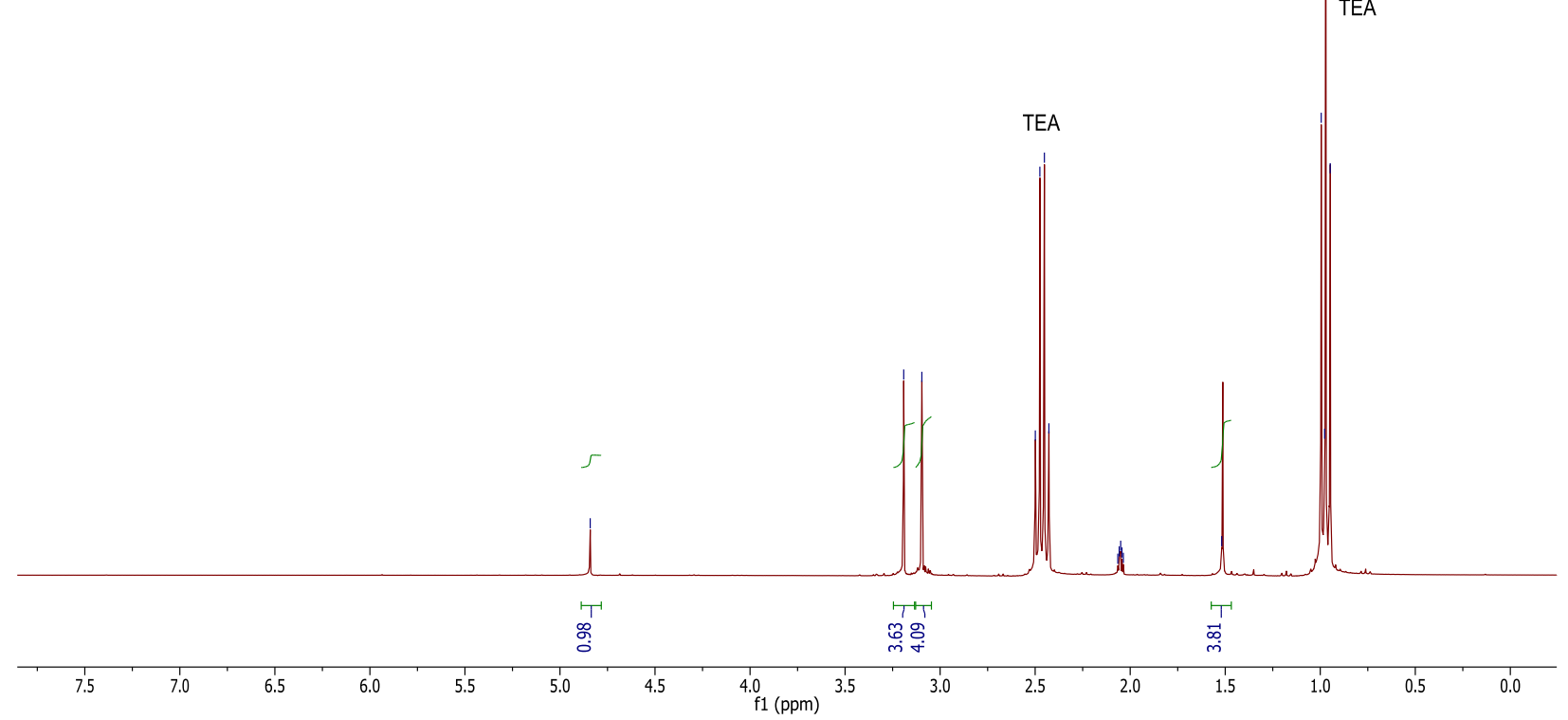

to

Compound 2

$\stackrel{\overrightarrow{\mathrm{N}}}{\mathrm{i}}$

㻤

TEA

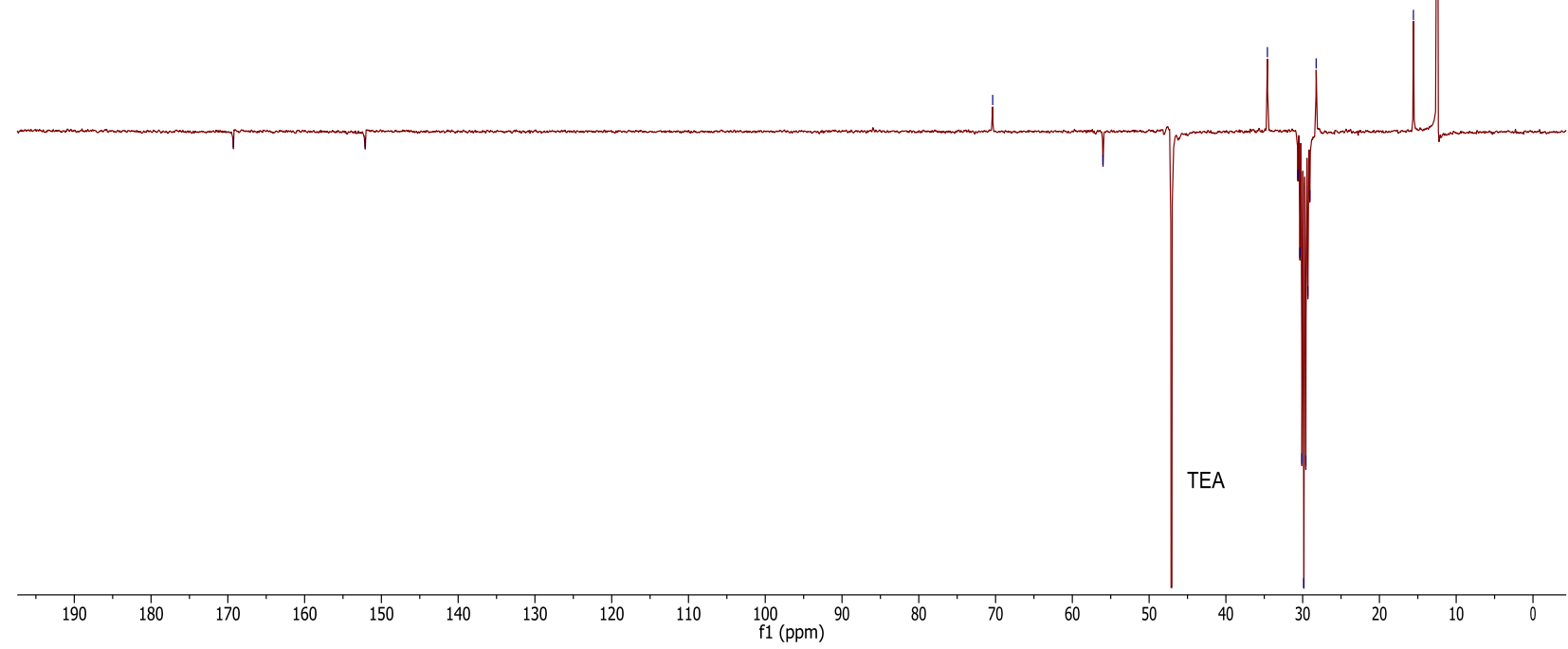


Compound $3 \stackrel{\frac{m}{8}}{3}$

管

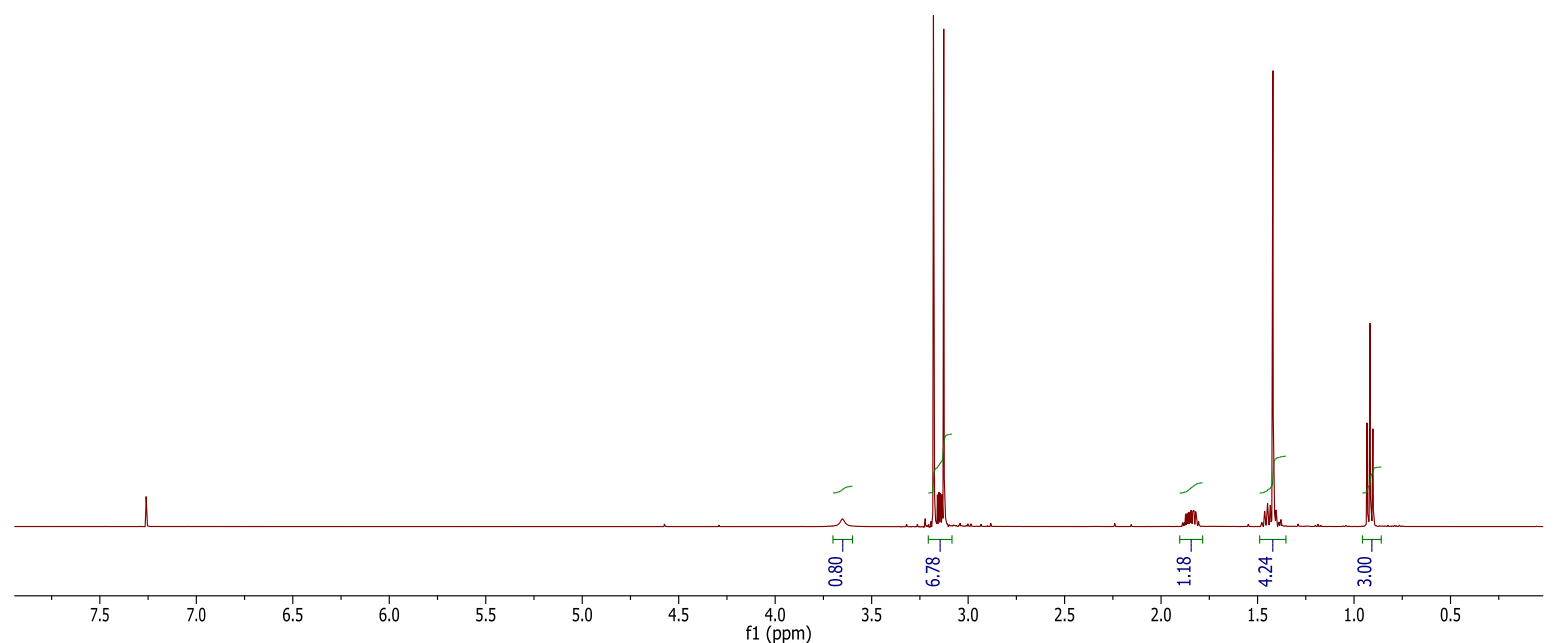

年

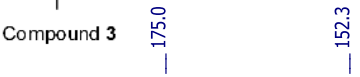

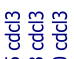

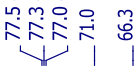

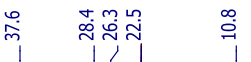

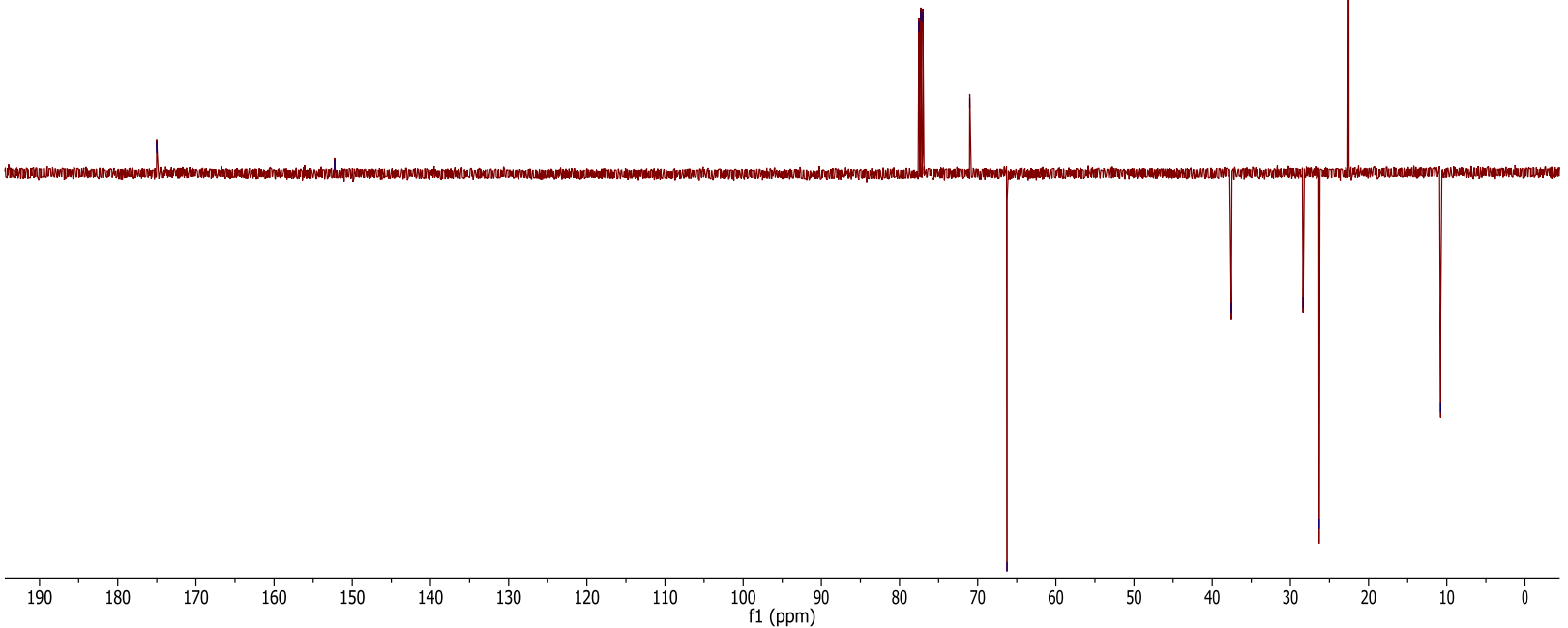




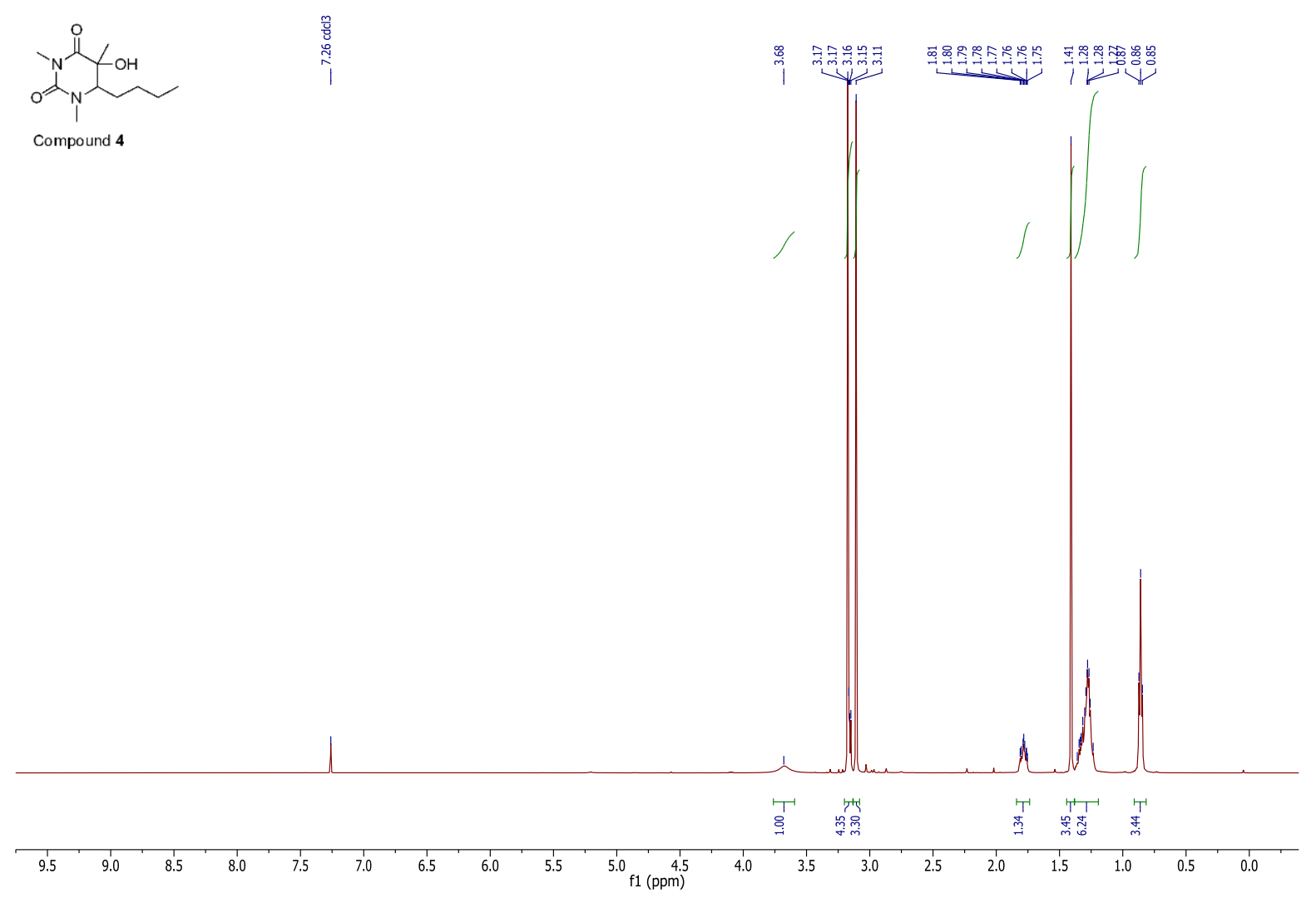

Compound 4

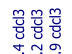

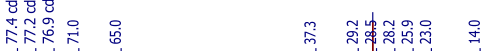

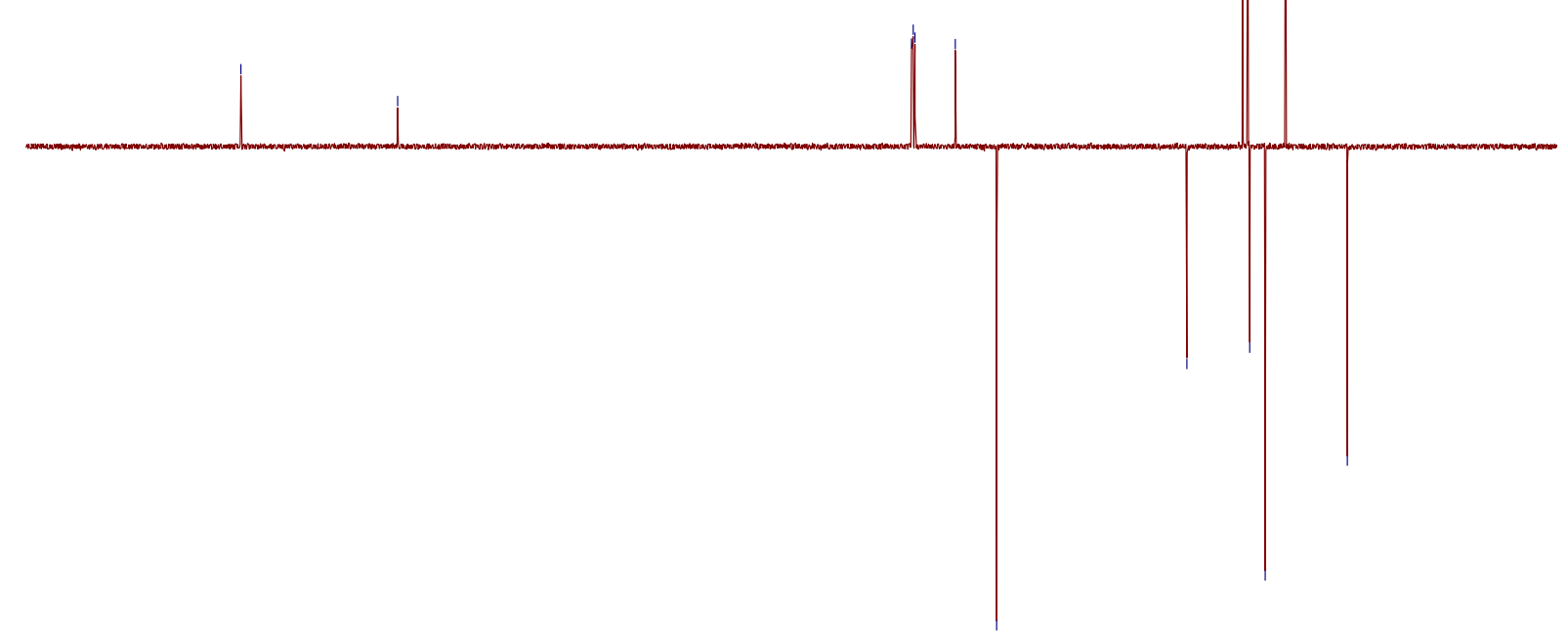

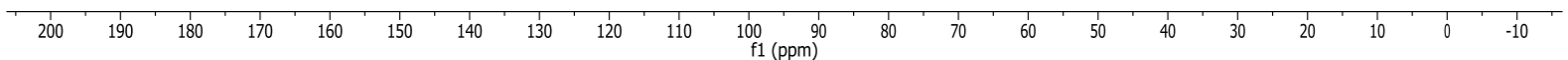




$$
\text { Compound } 5
$$

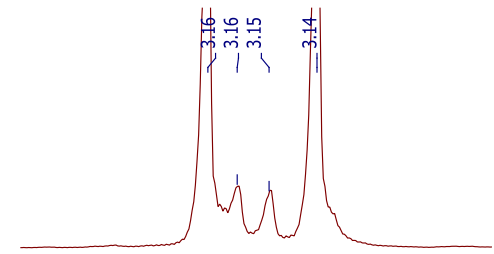

$\begin{array}{llllllllll}3.20 & 3.19 & 3.18 & 3.17 & 3.16 & 3.15 & 3.14 & 3.13 & 3.12 & 3.11 \\ \text { f1 (ppm) } & 3.14 & & & \end{array}$

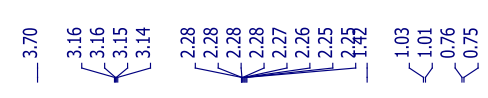

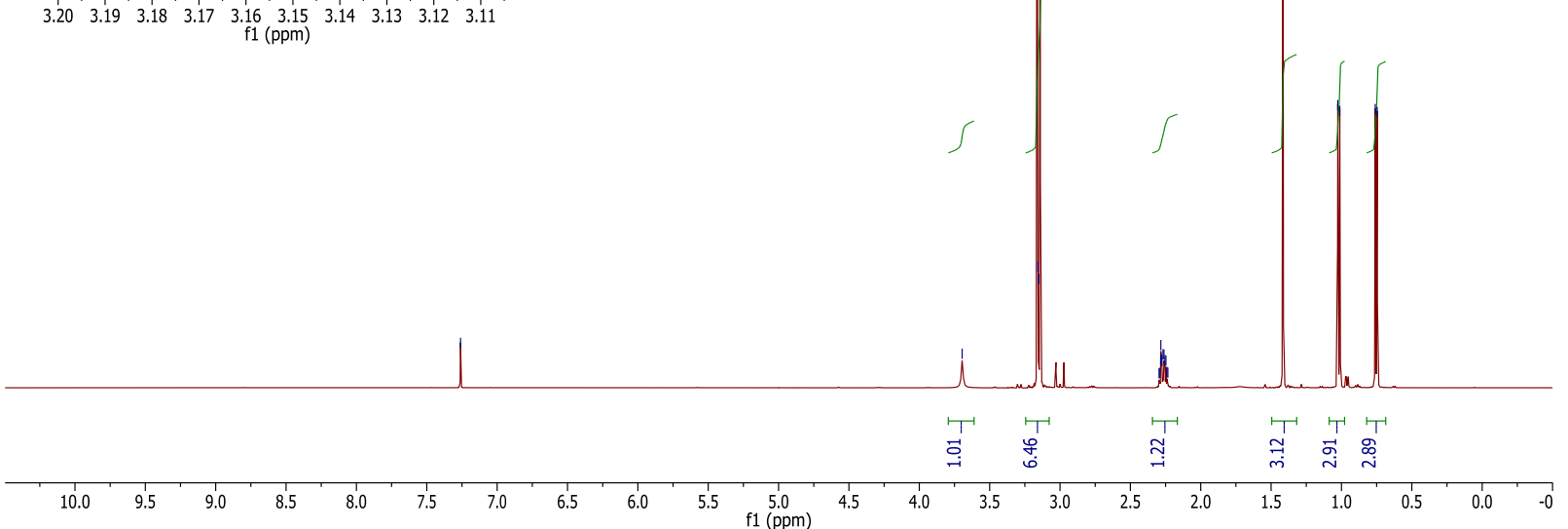

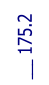

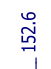

을

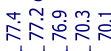

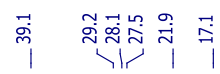

(1)

Compound 5

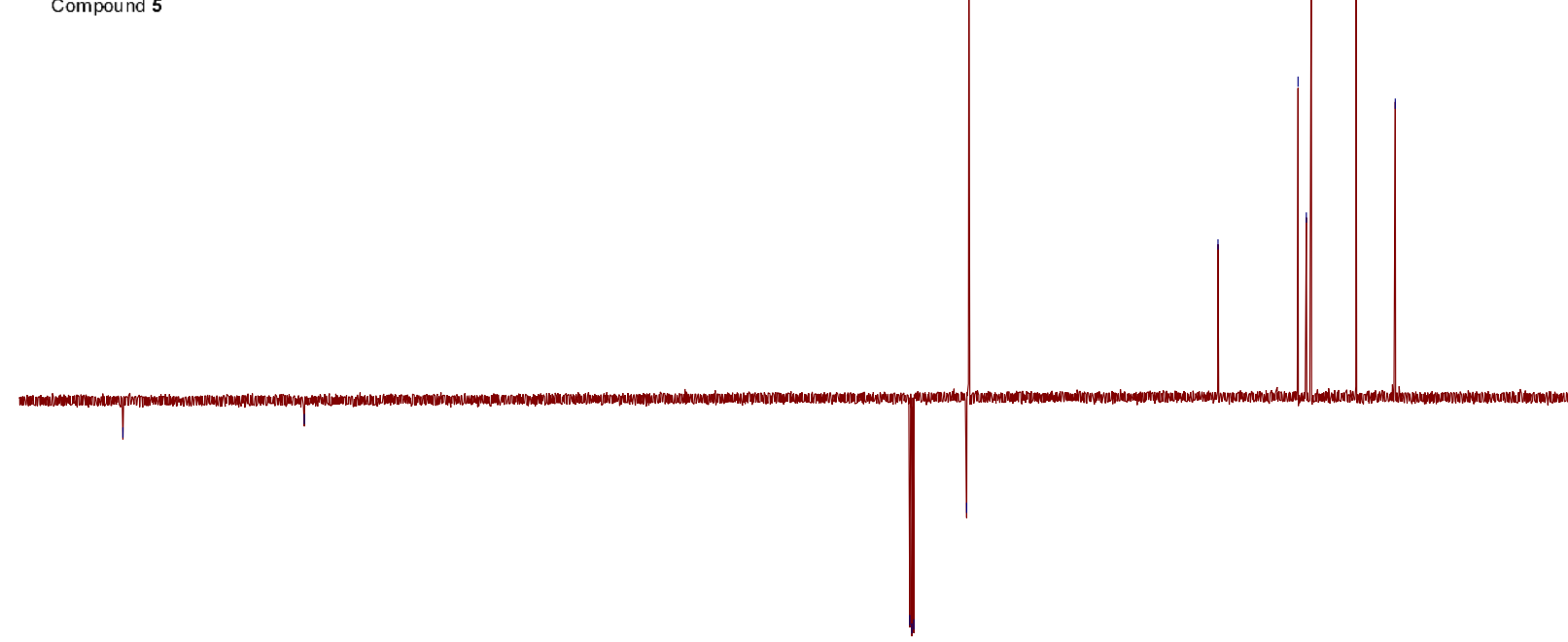

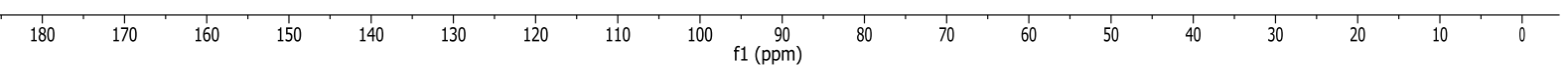



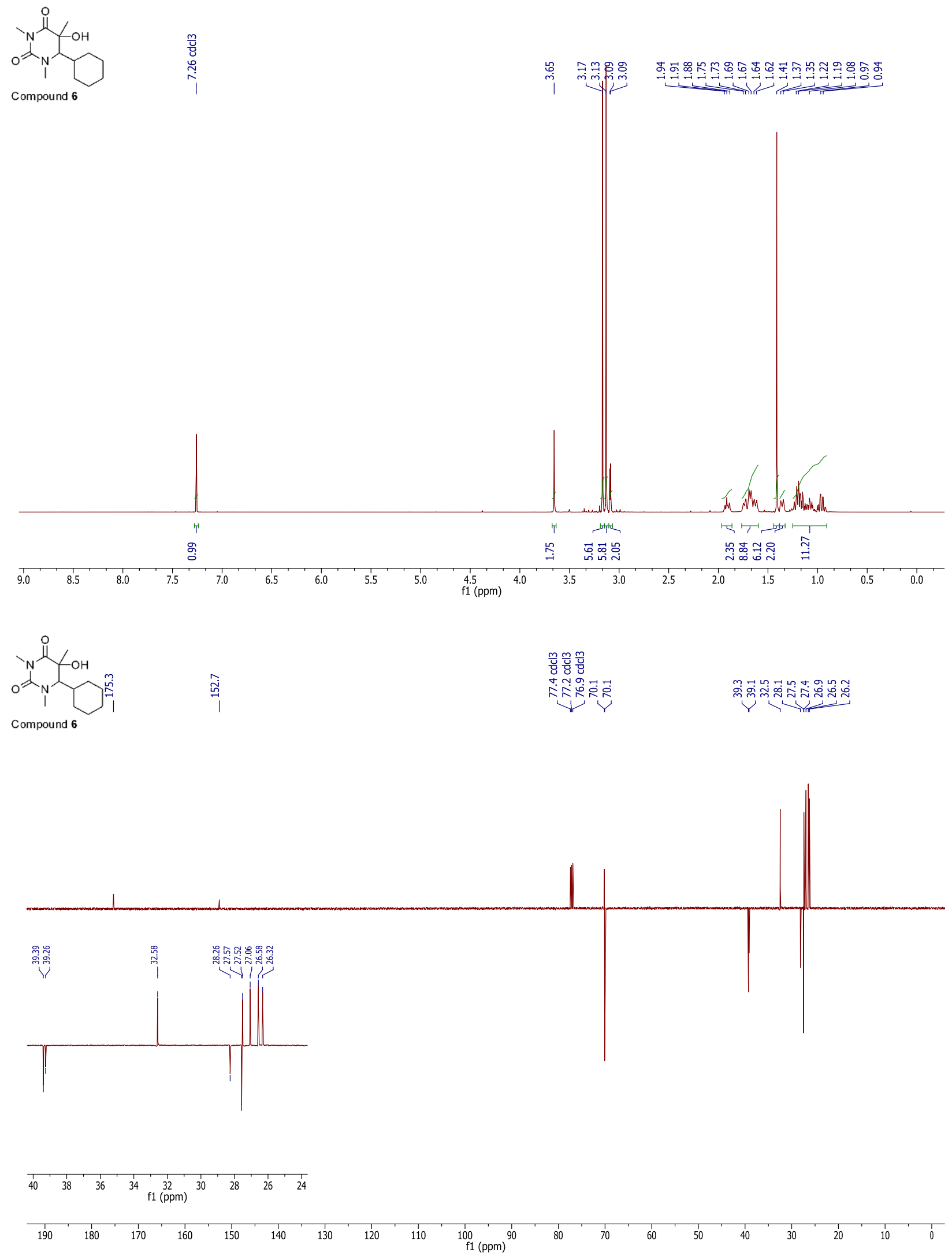

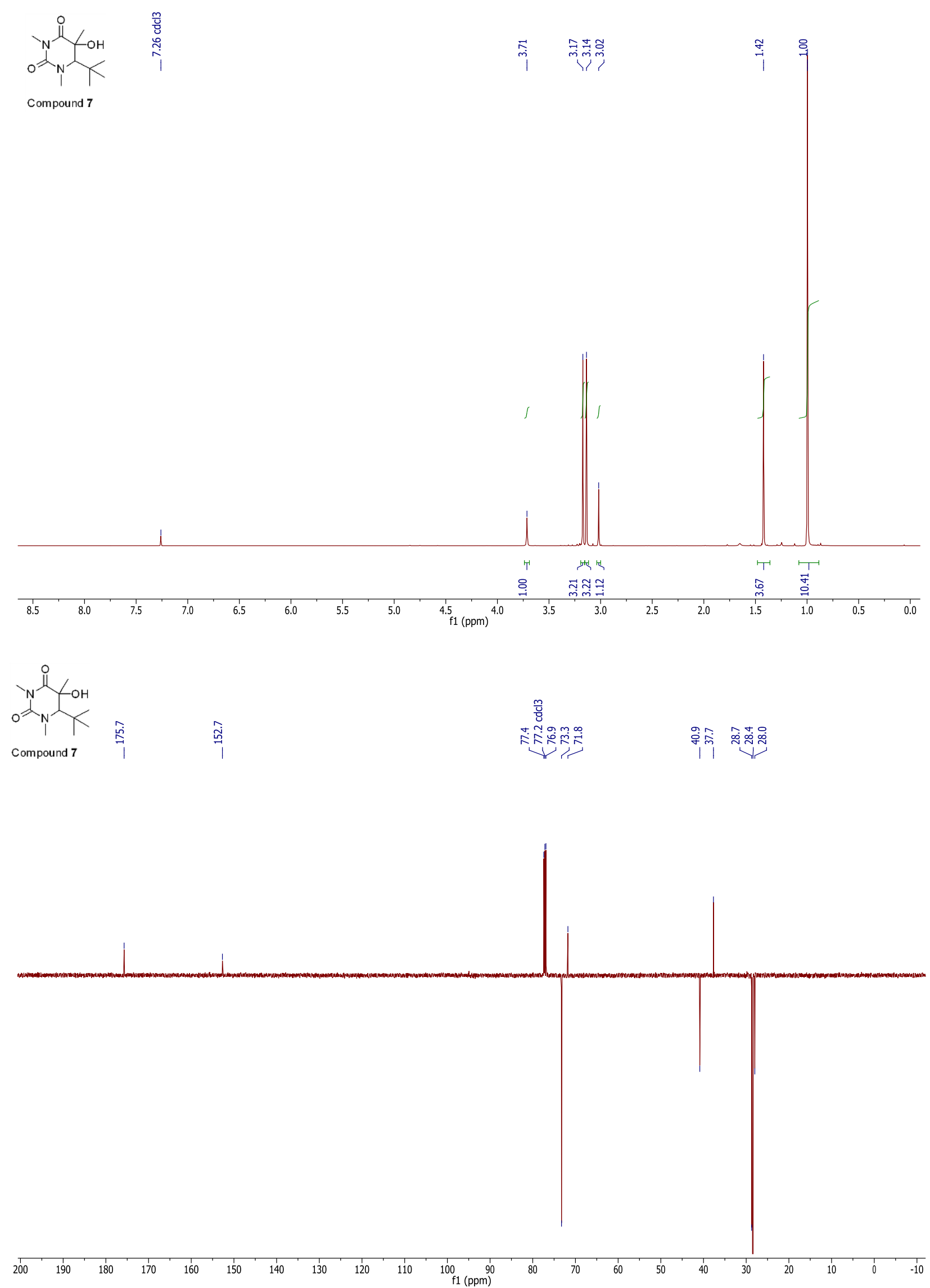

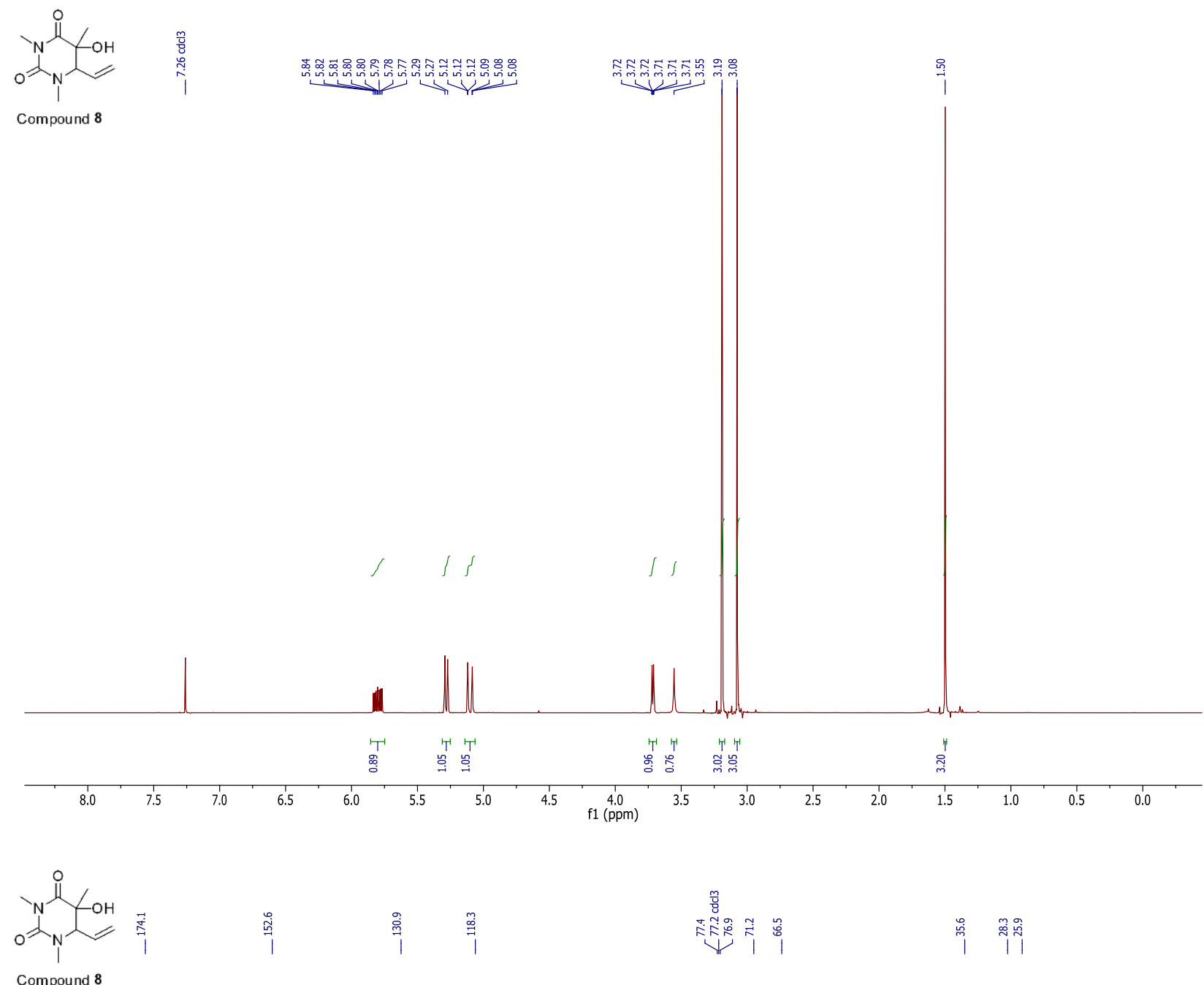

Compound 8

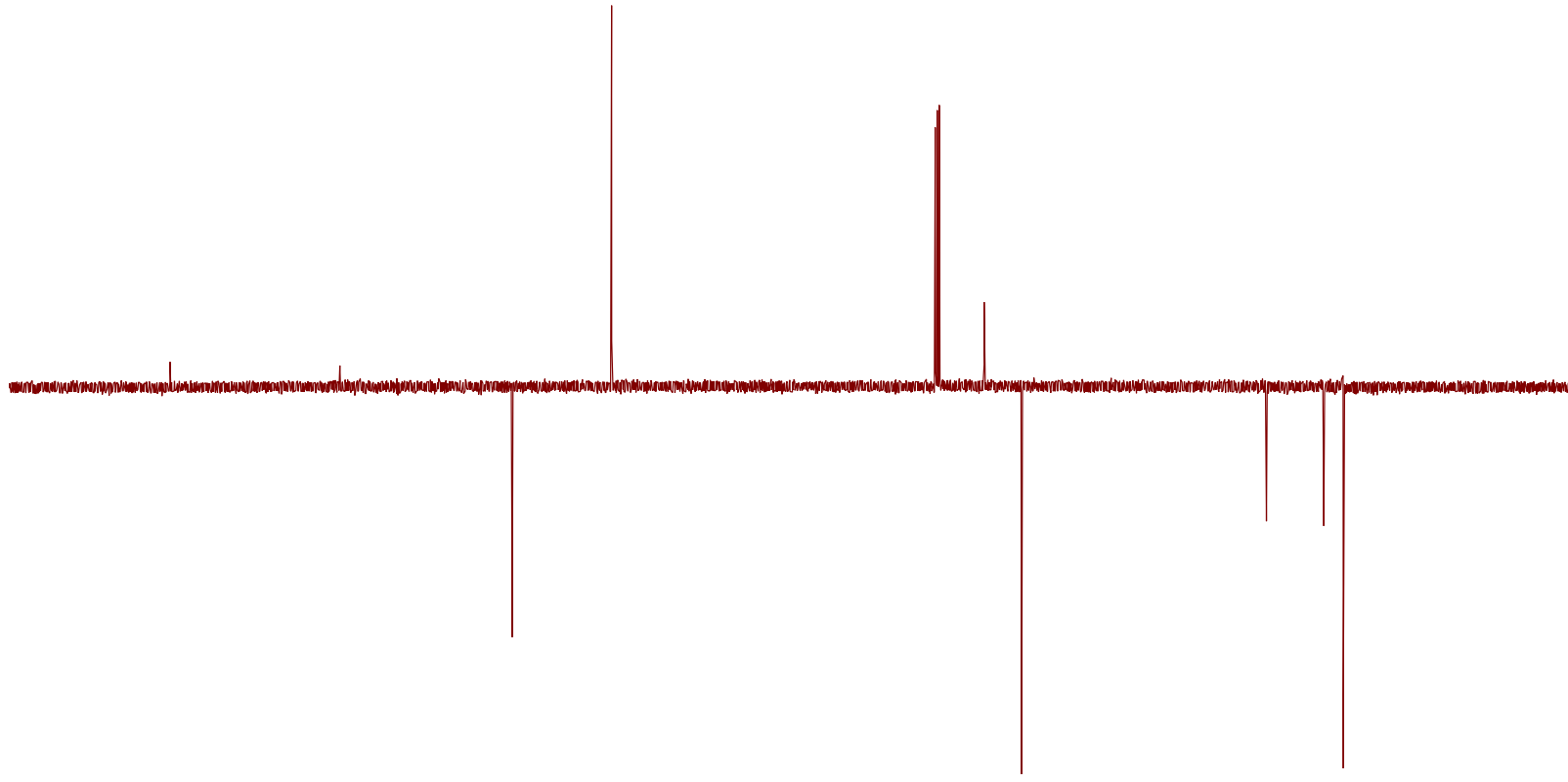

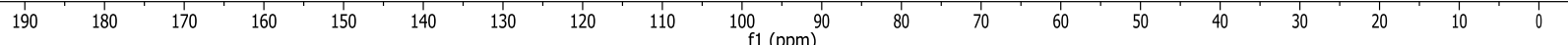



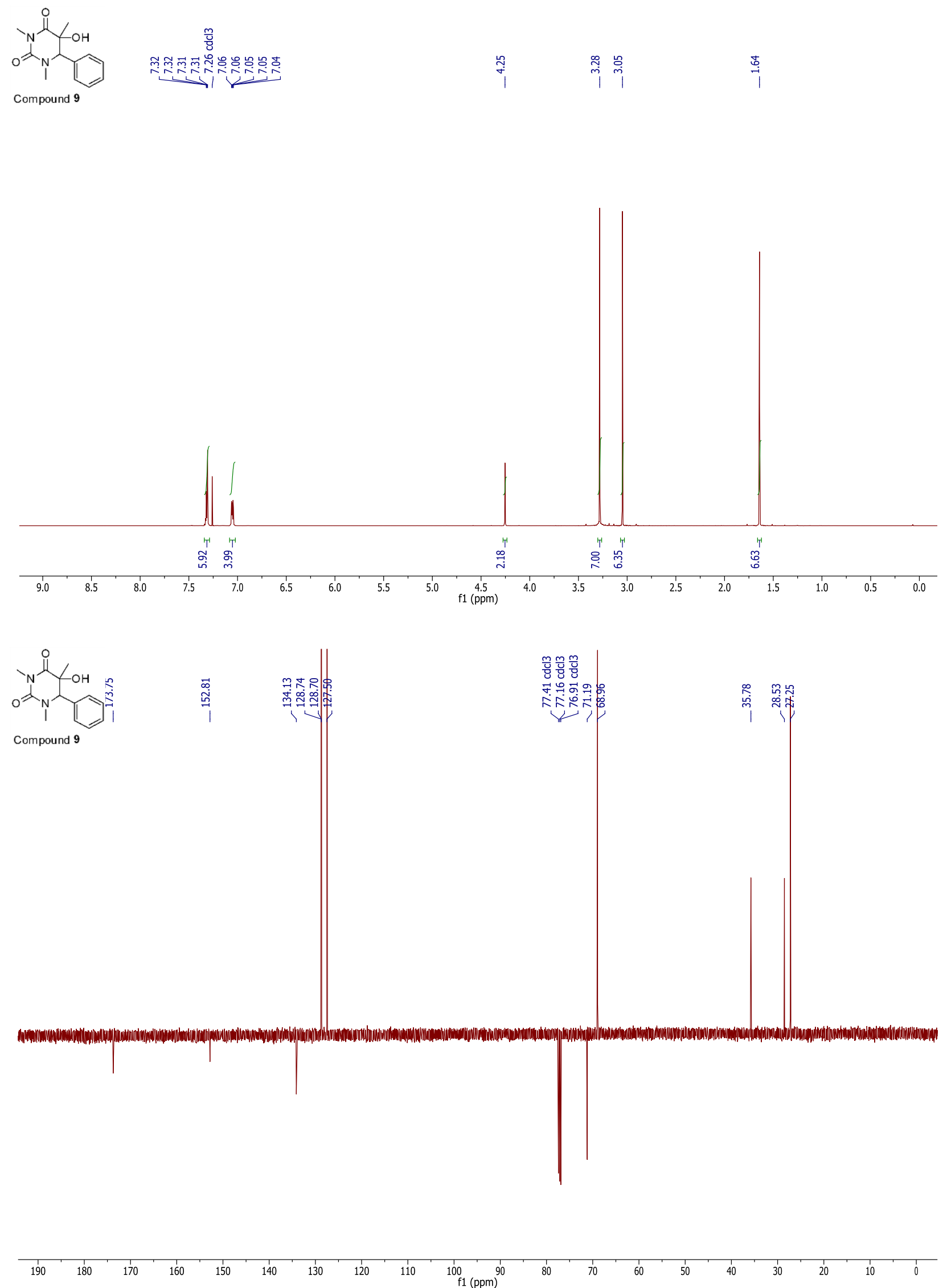


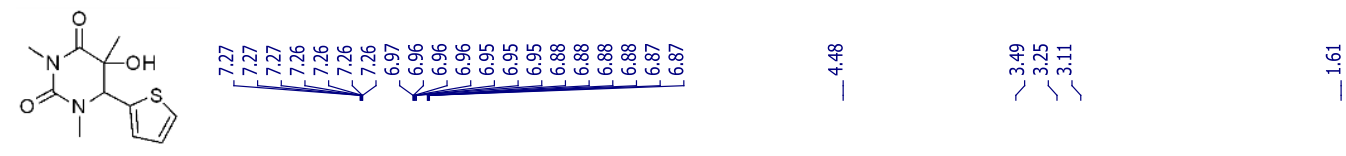

Compound 10
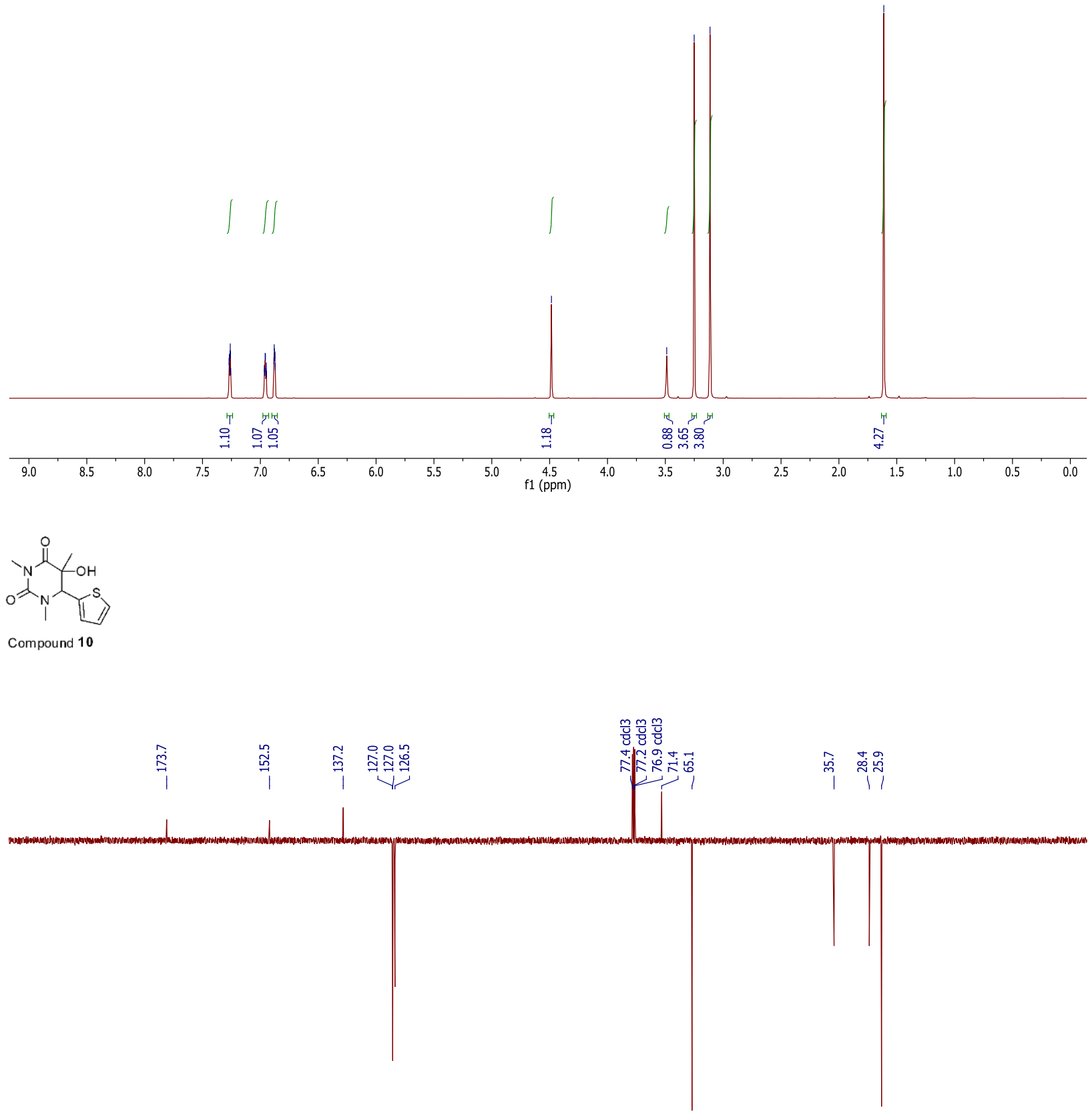

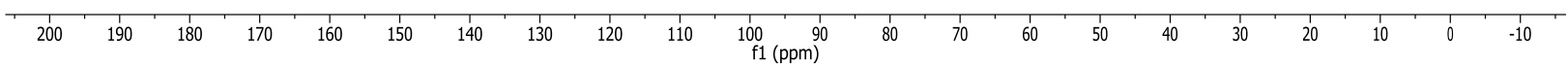


Compound 11

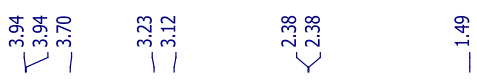

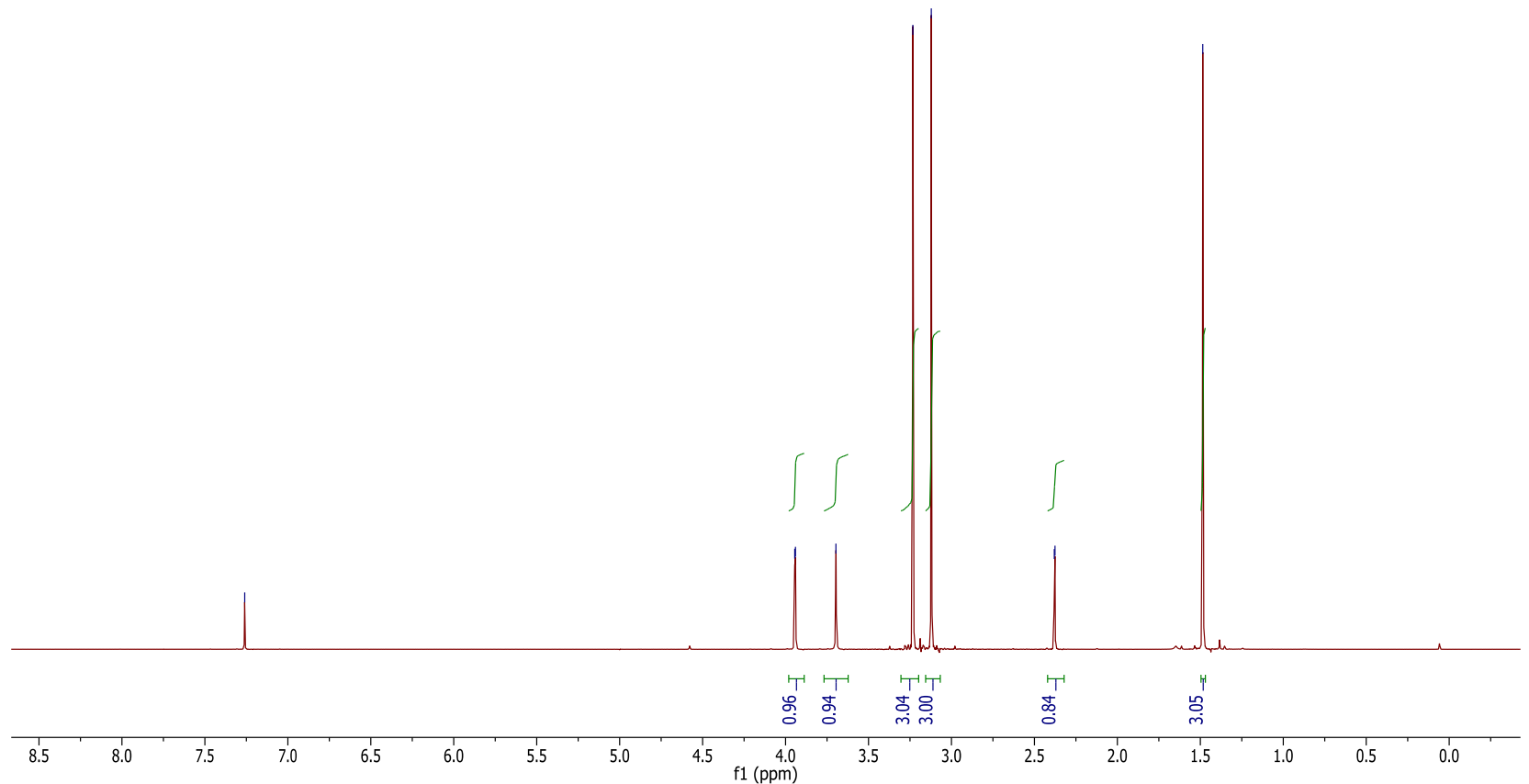

䎡

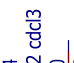

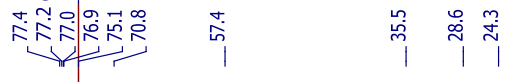

Compound 11

(1)
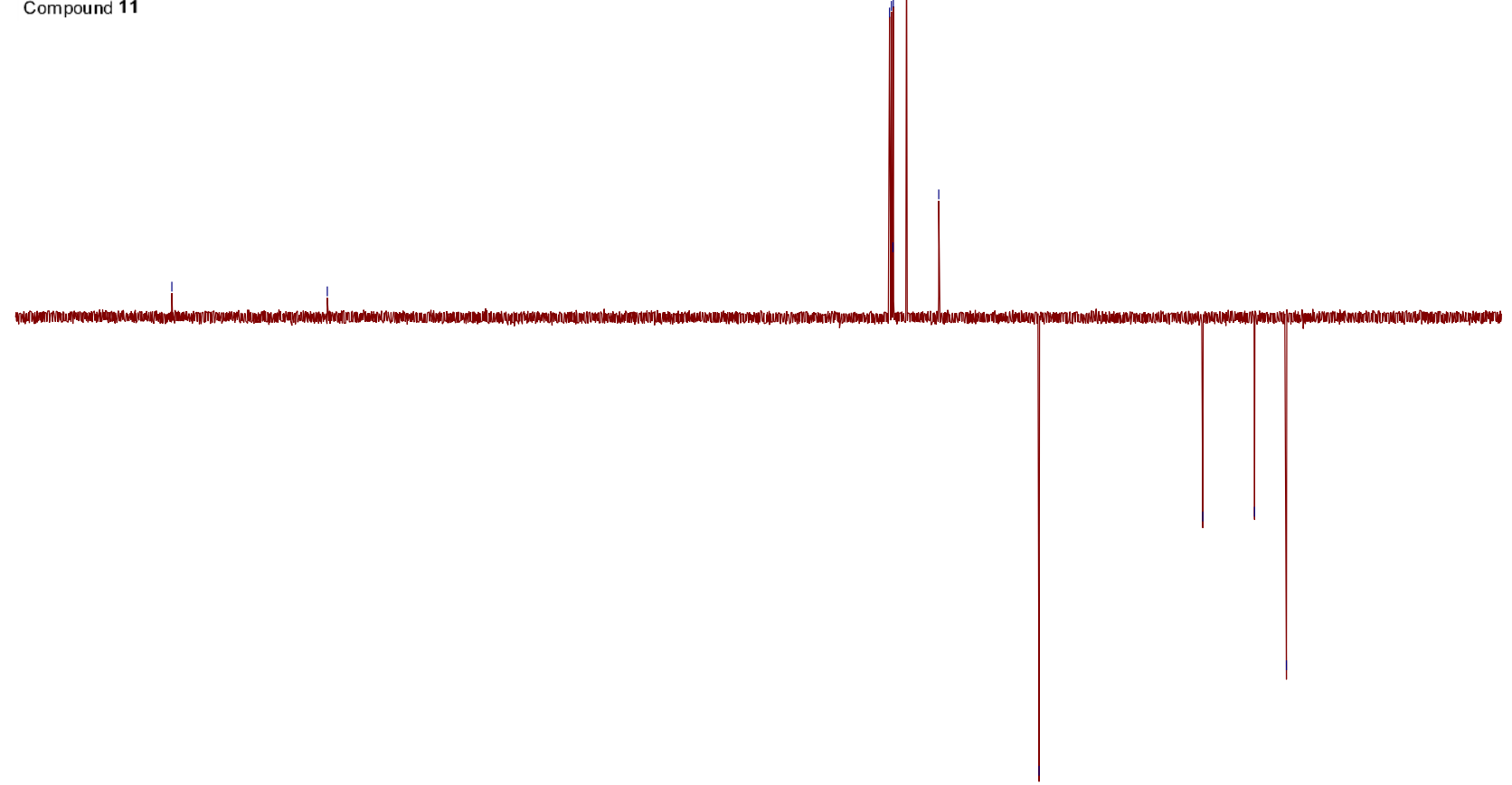

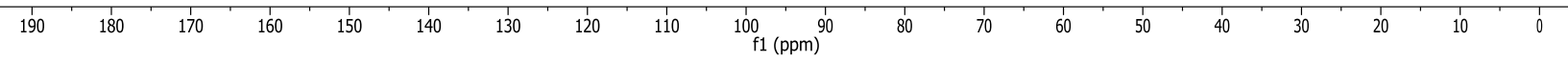



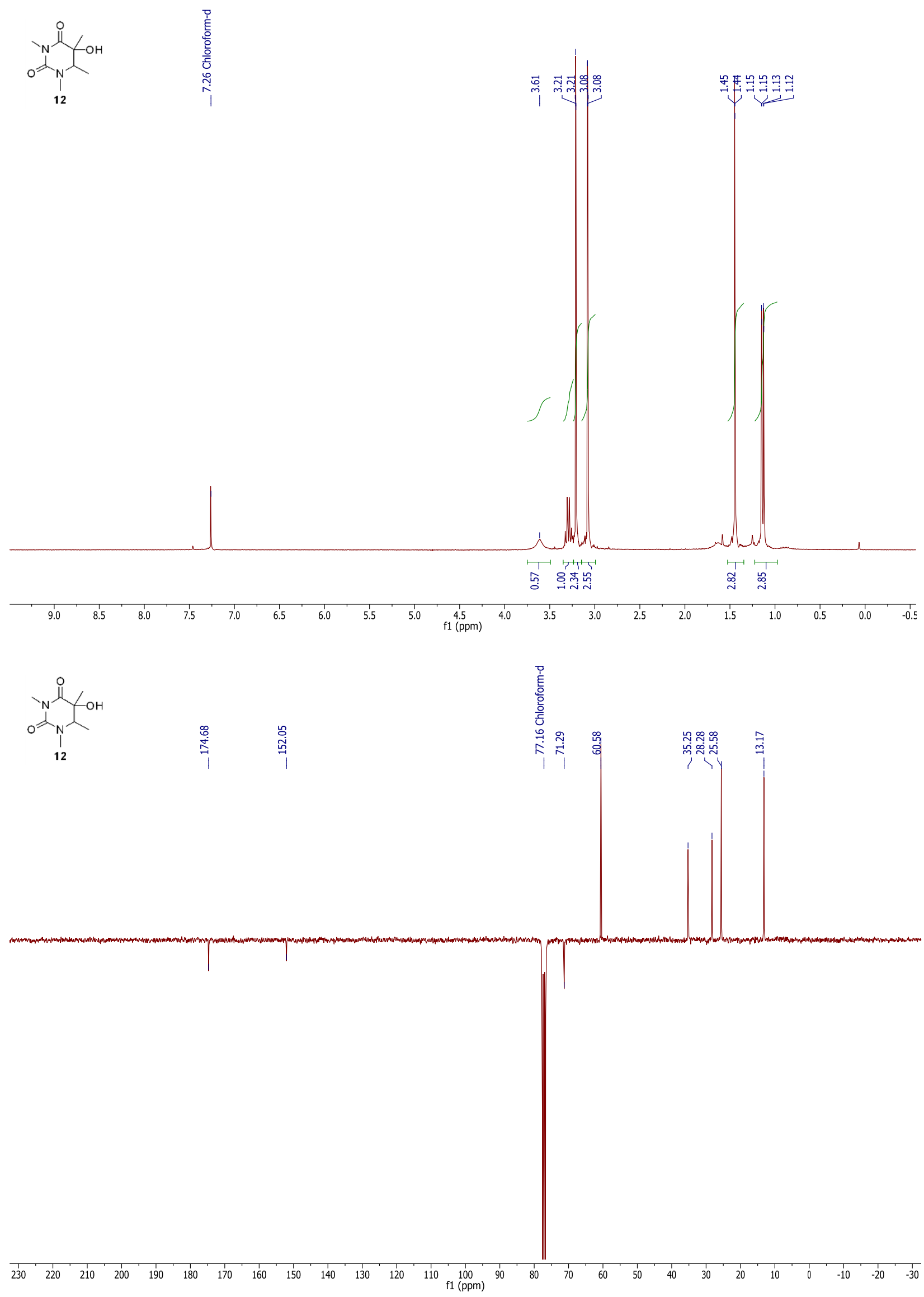

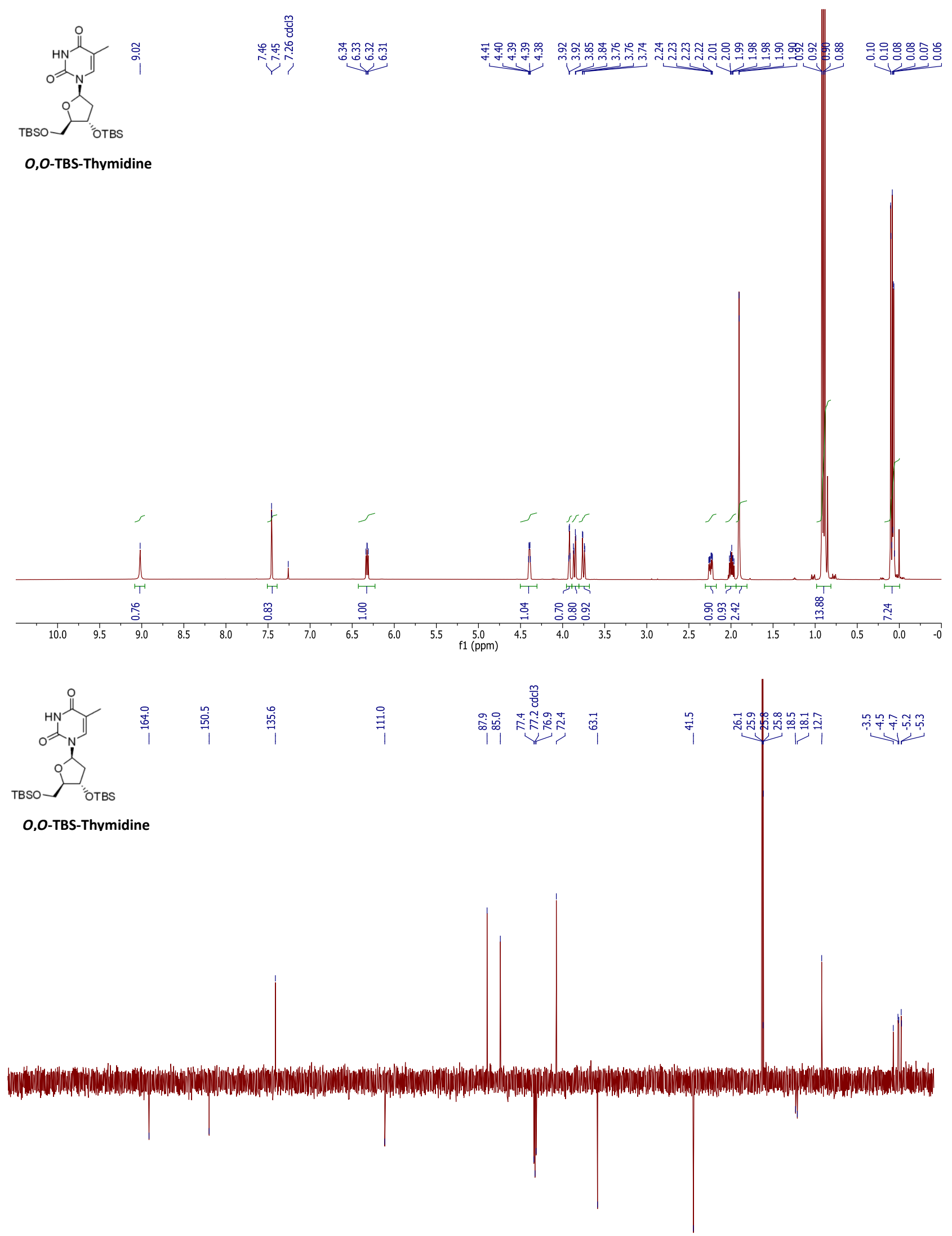

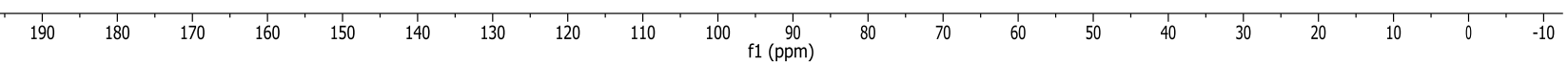



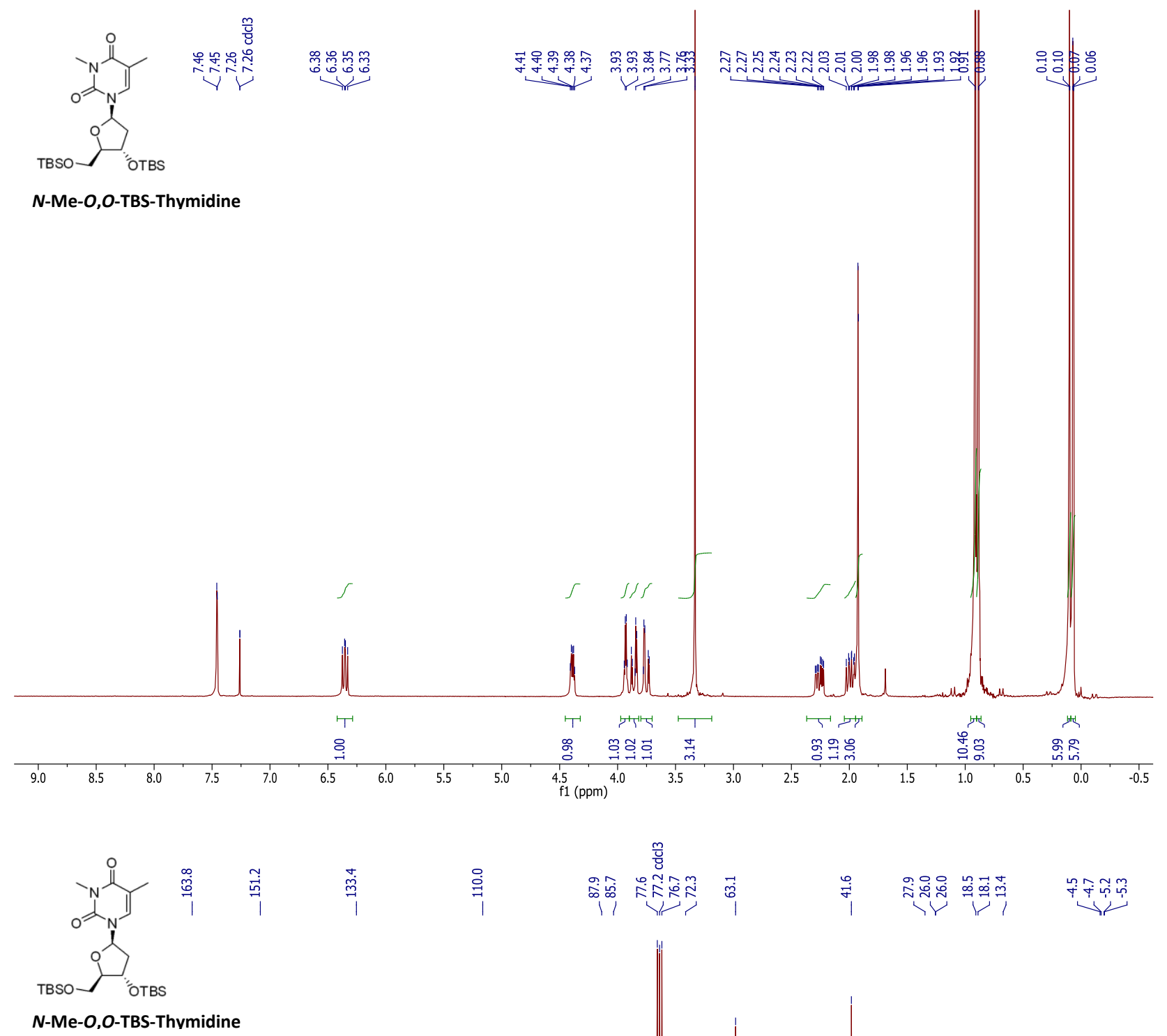

N-Me-O,O-TBS-Thymidine

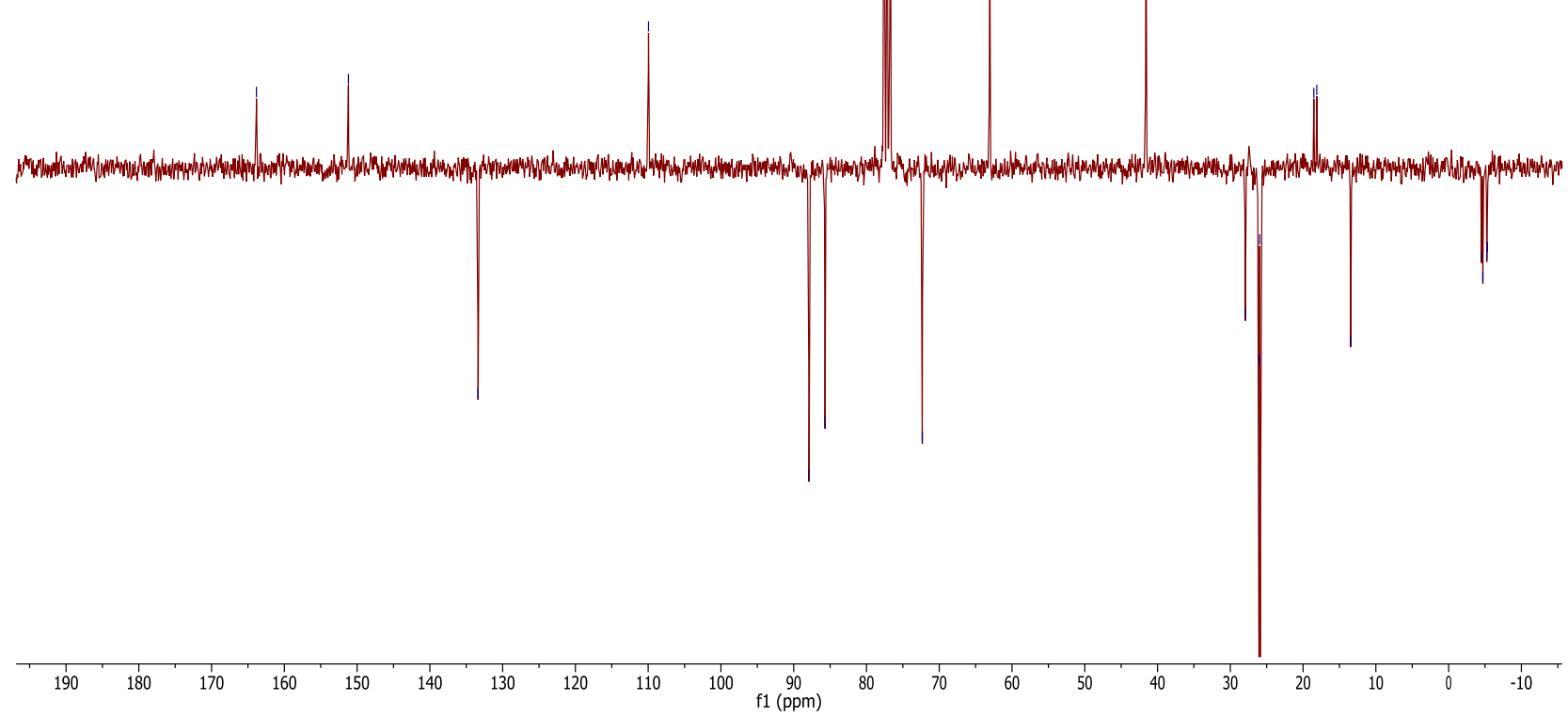



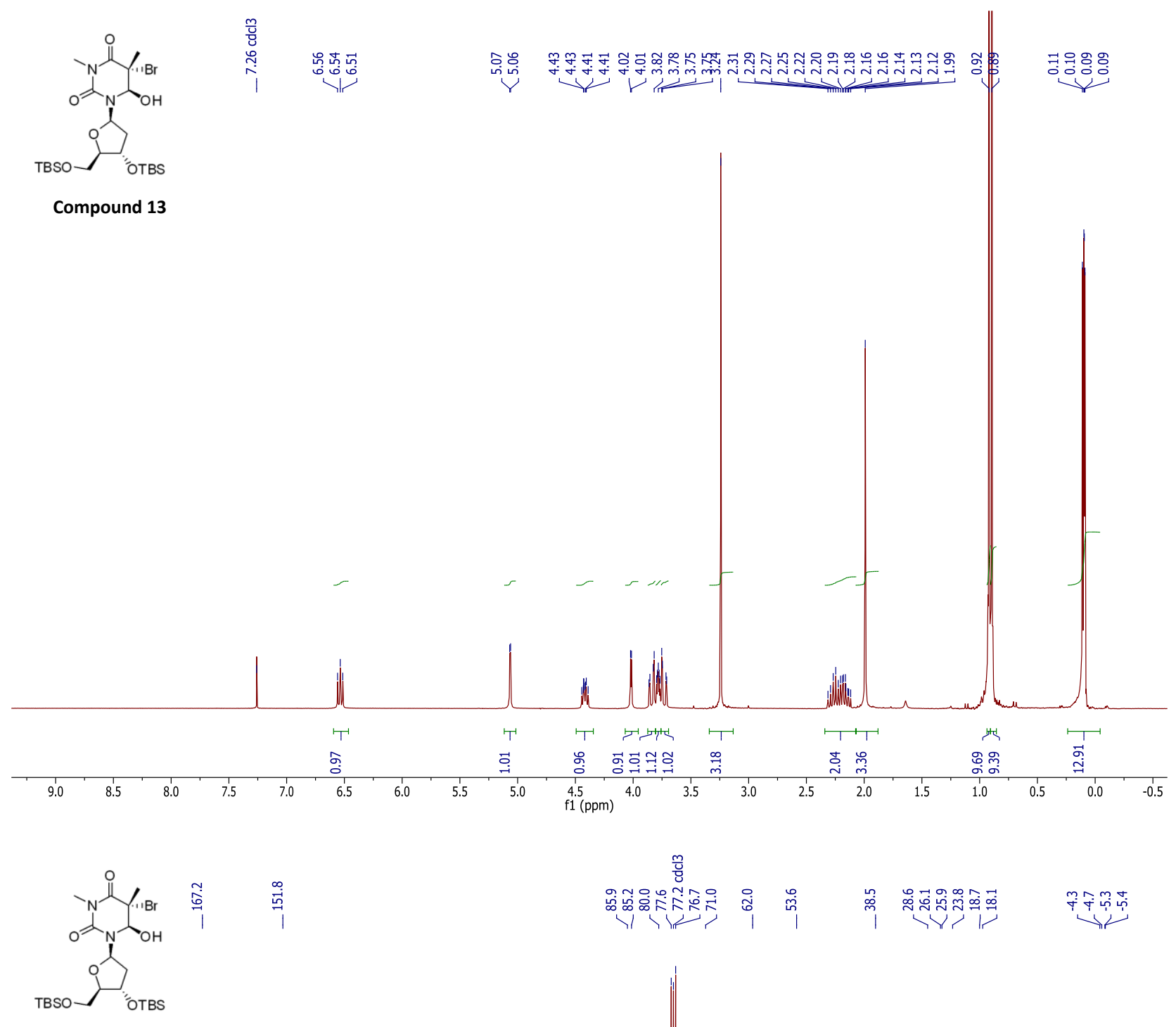

Compound 13

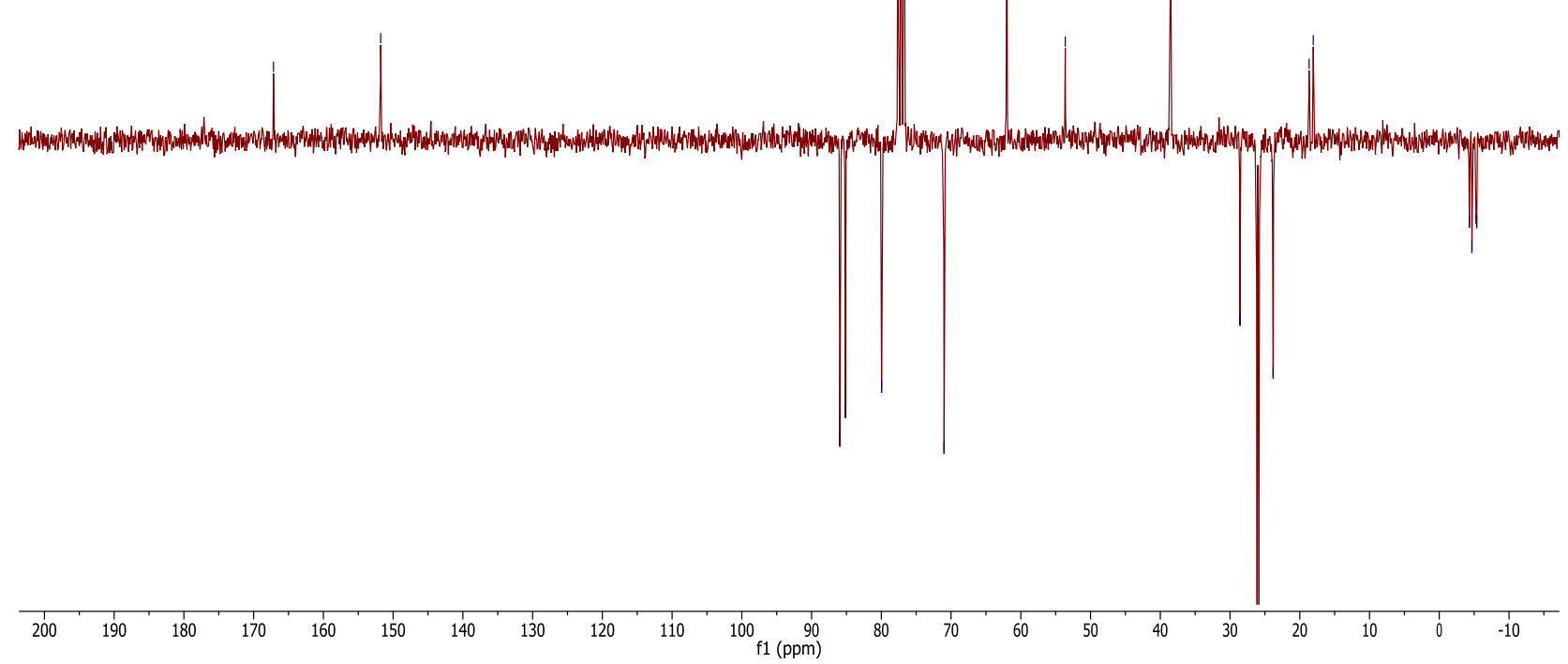



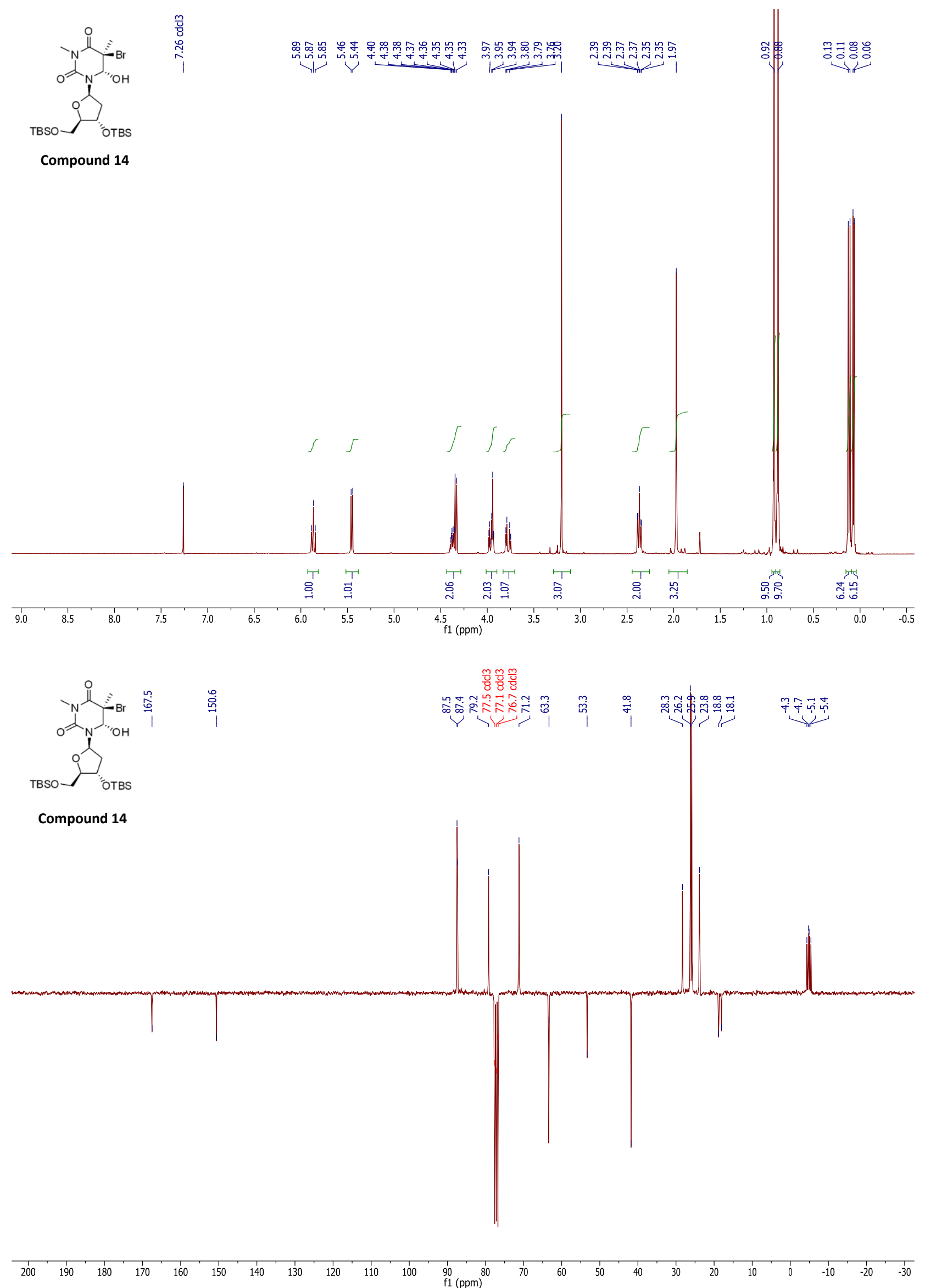


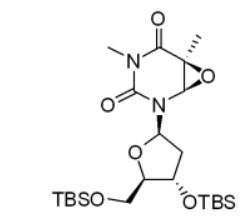

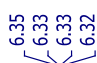

Compound 15
| $\underbrace{\infty} \underbrace{\infty}$
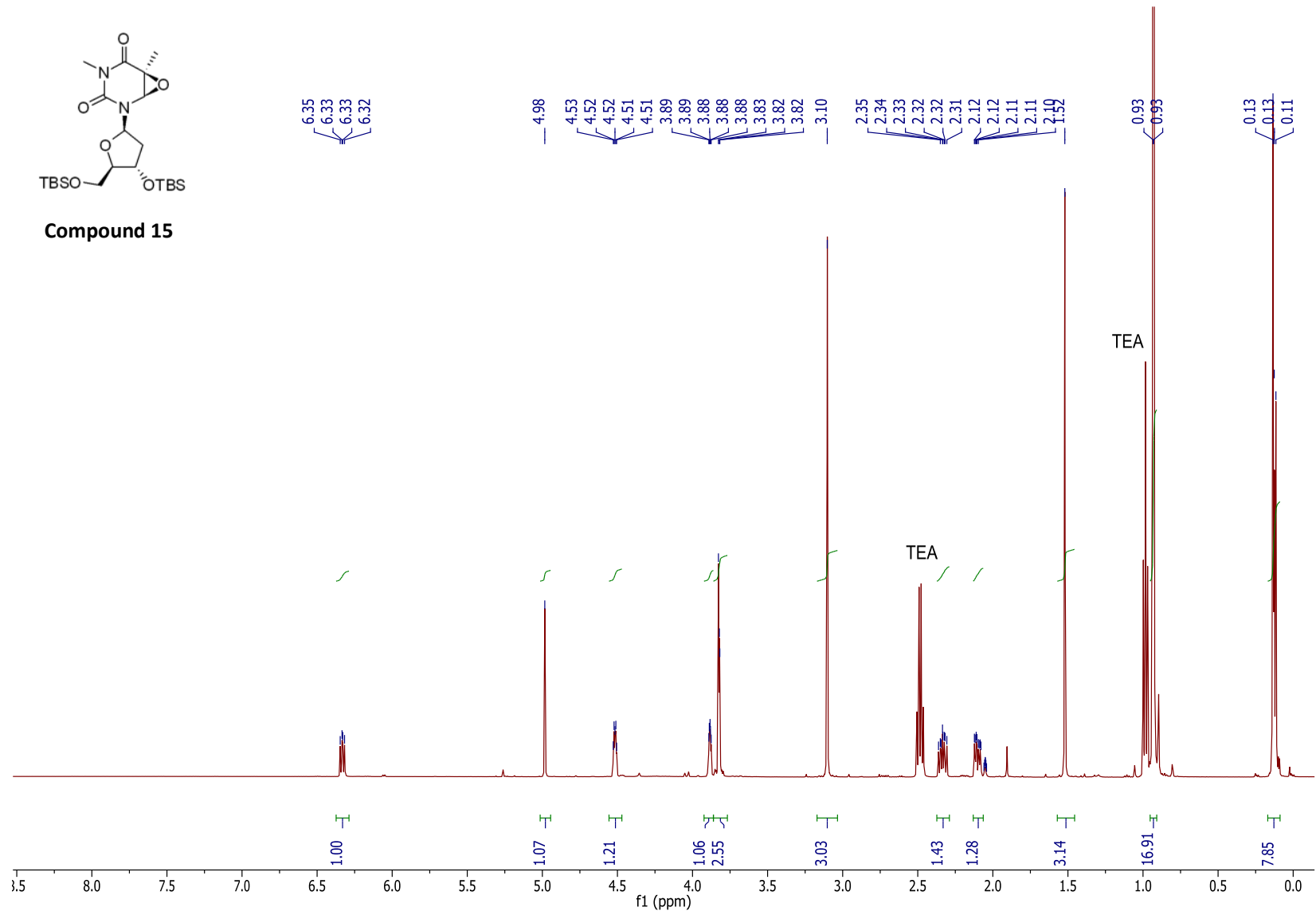

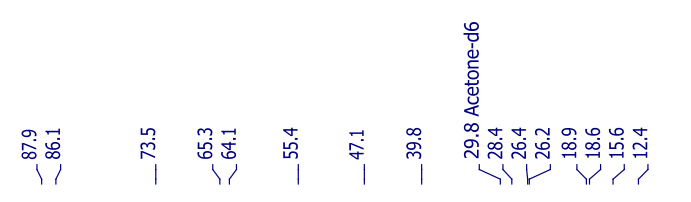

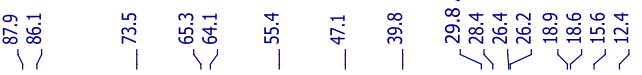

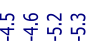

Compound 15

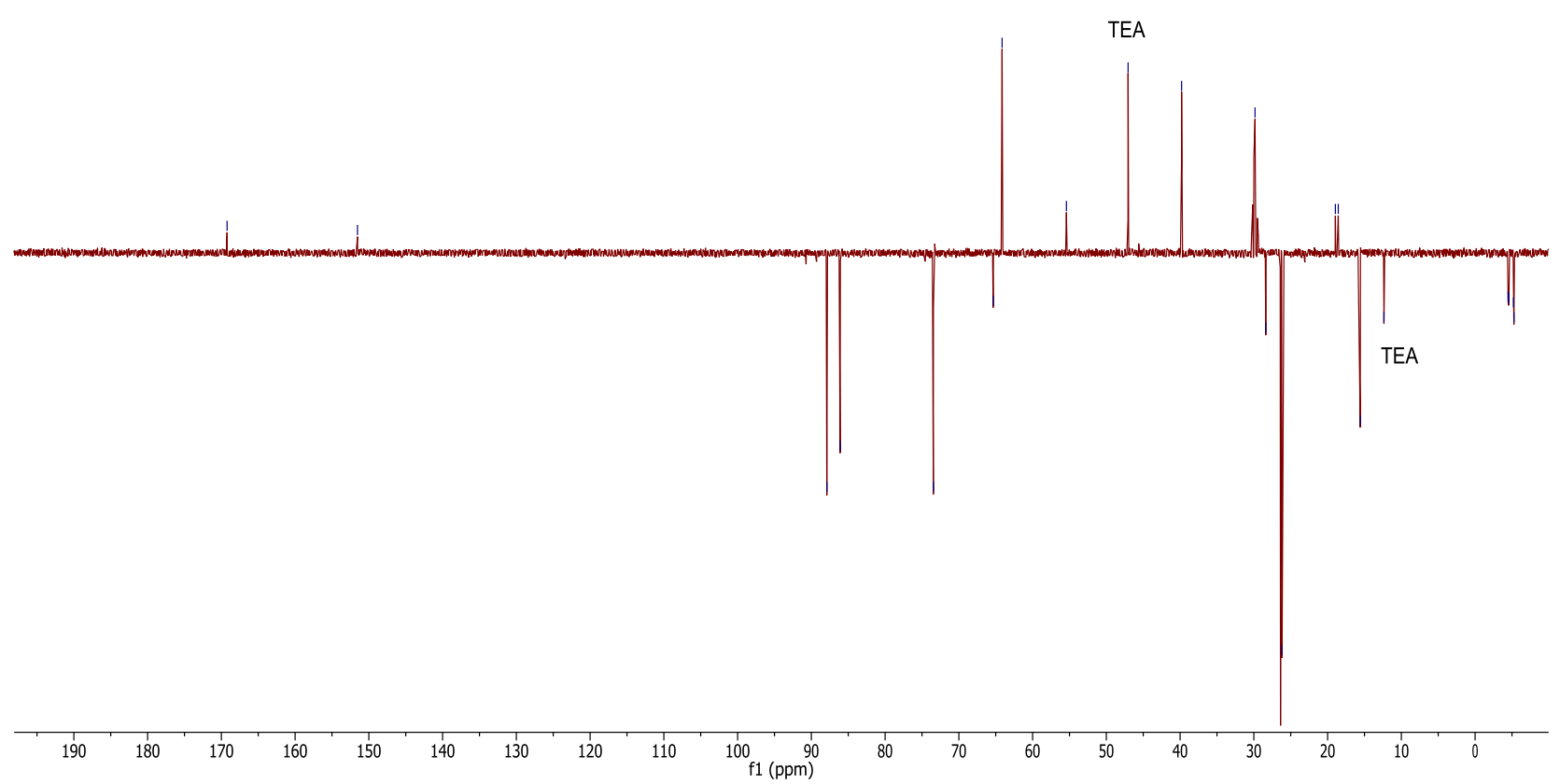




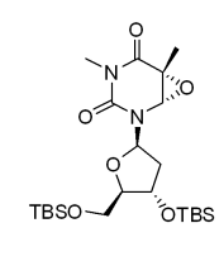

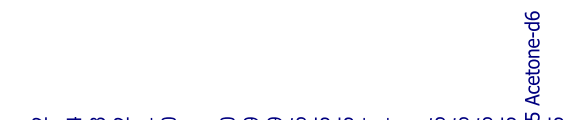

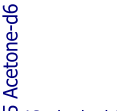

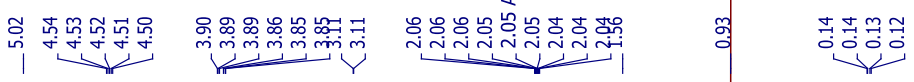

Compound 16
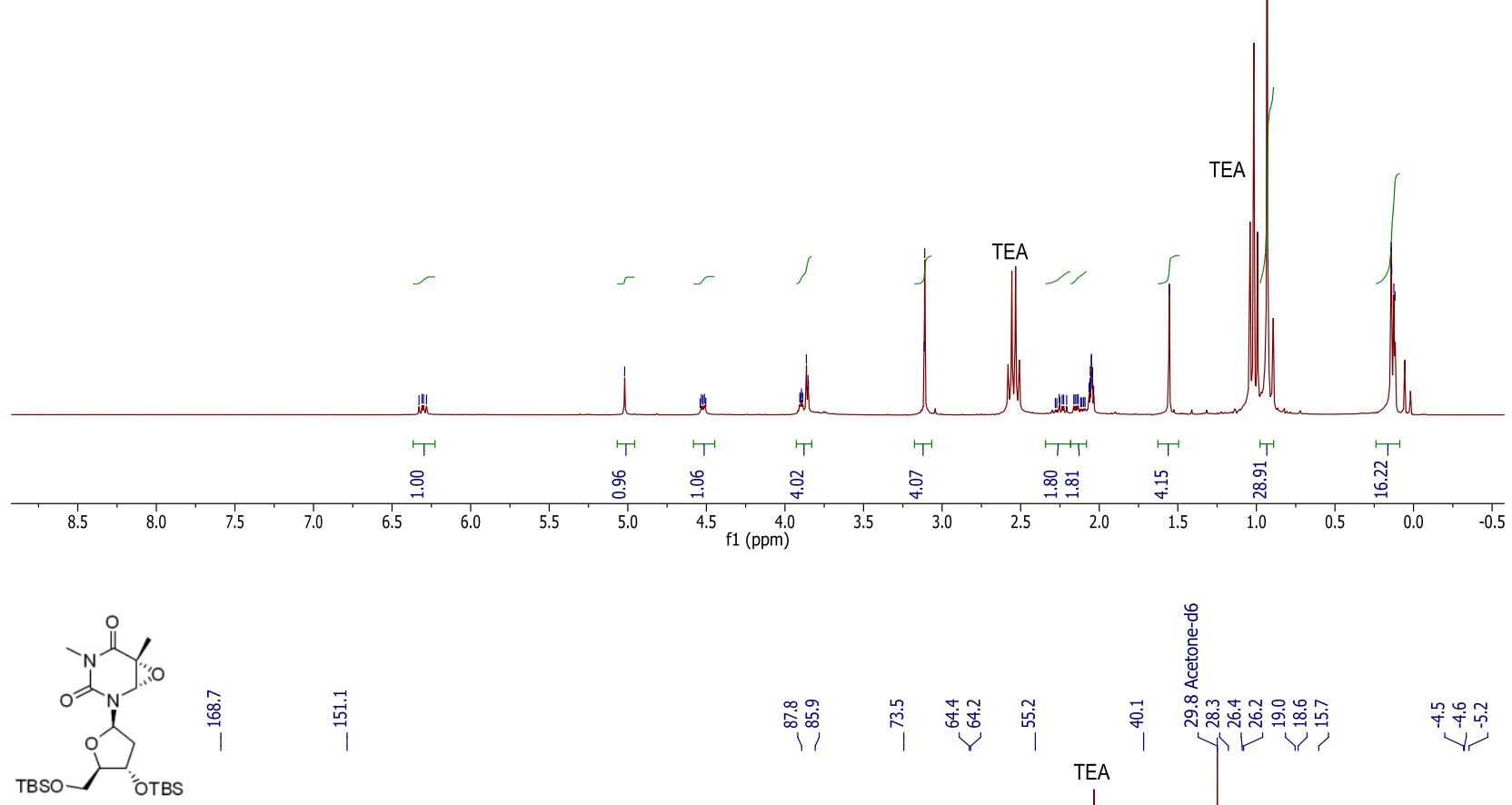

Compound 16

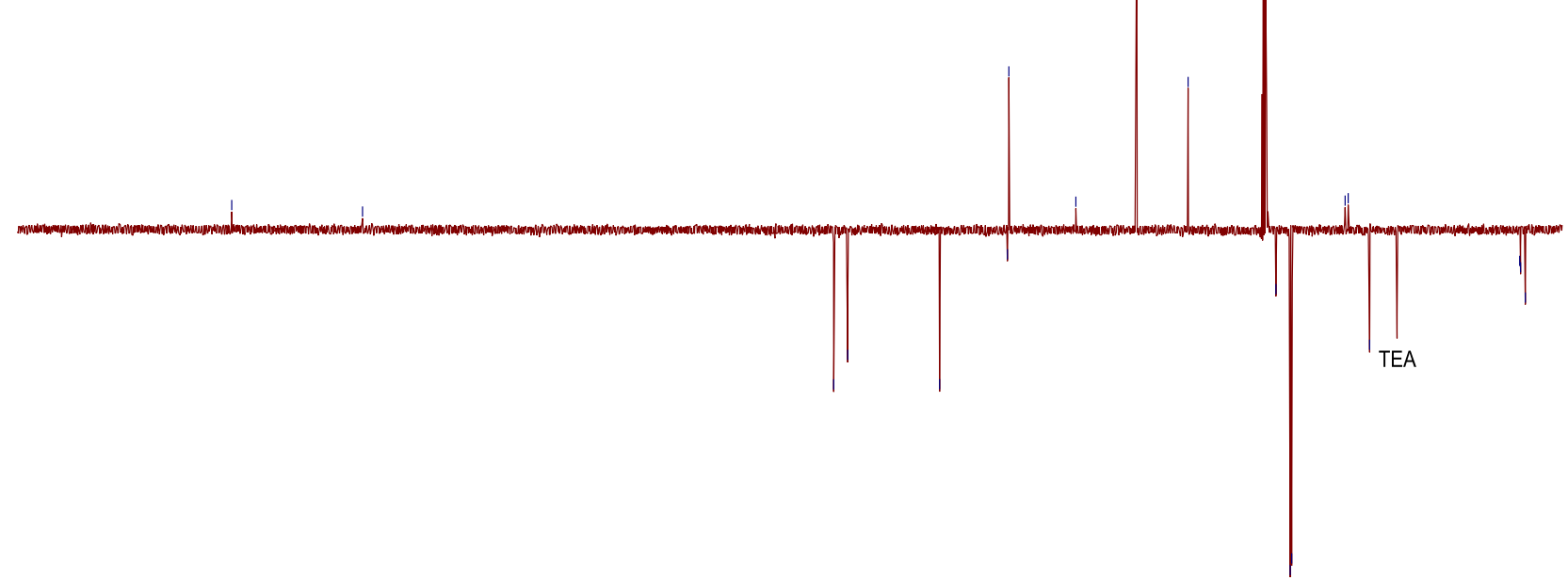

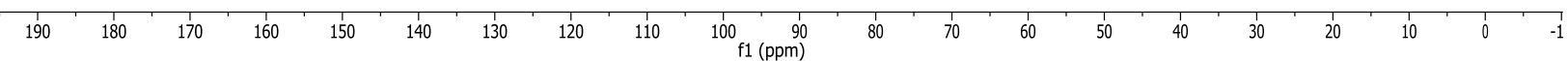



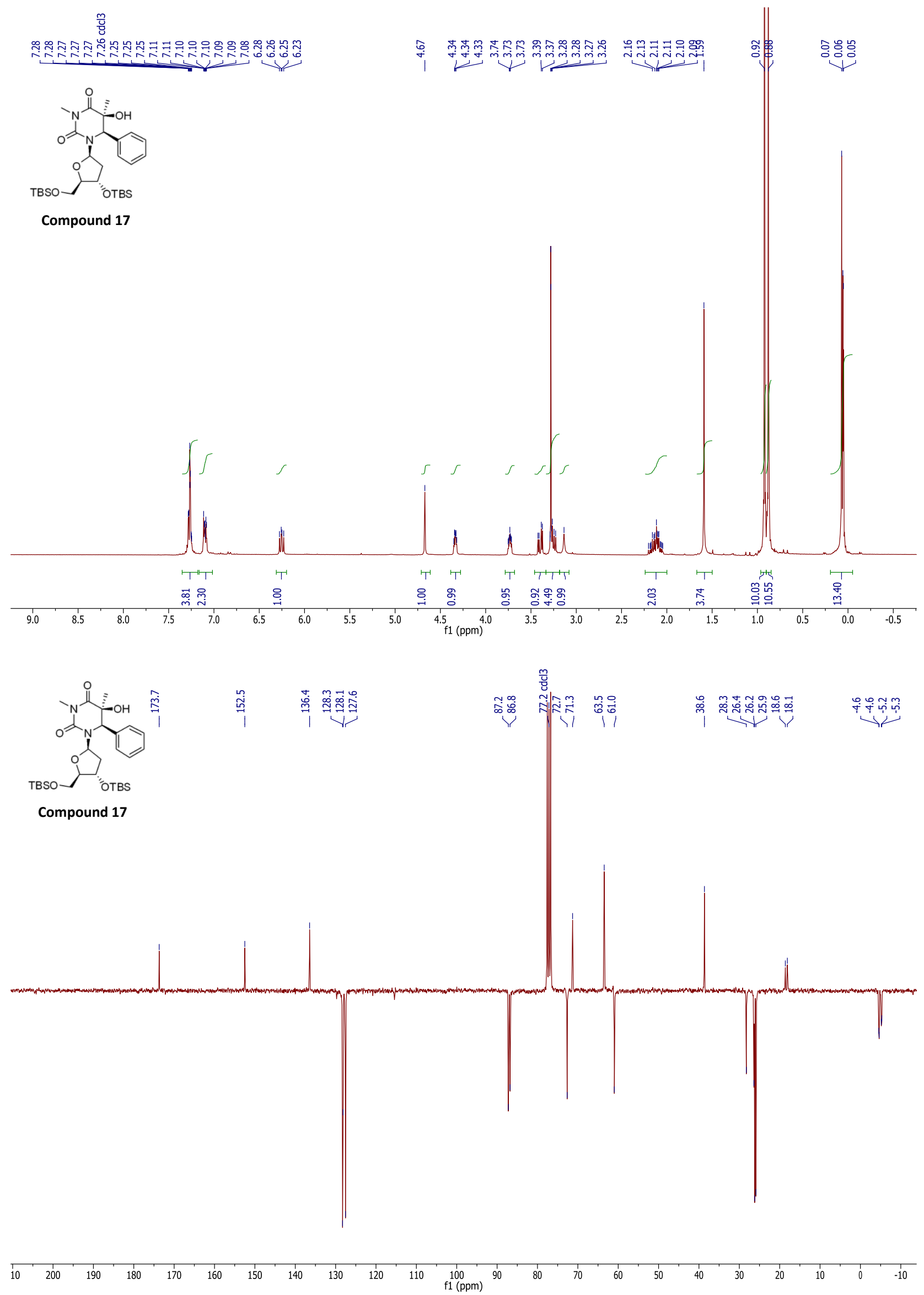

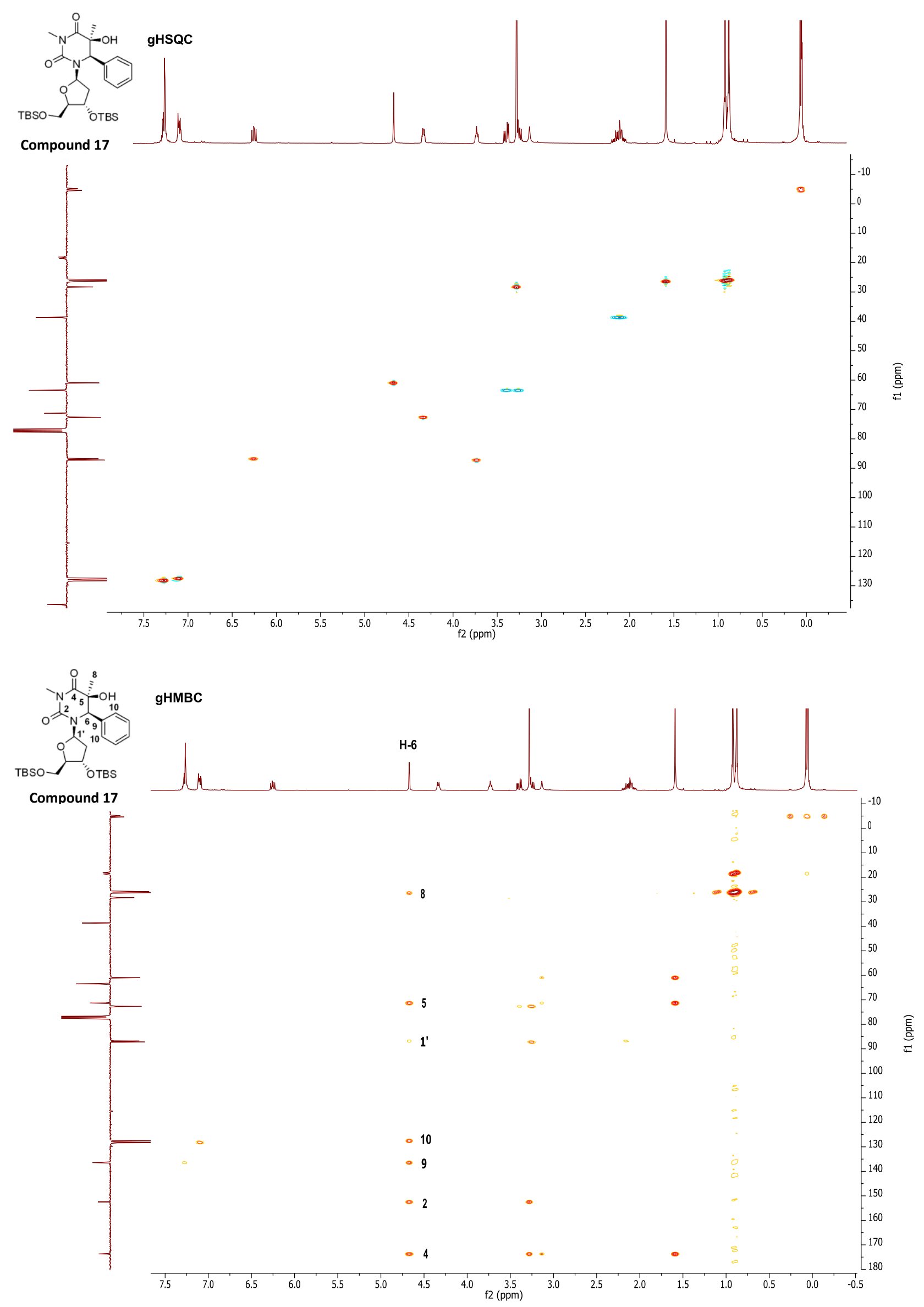


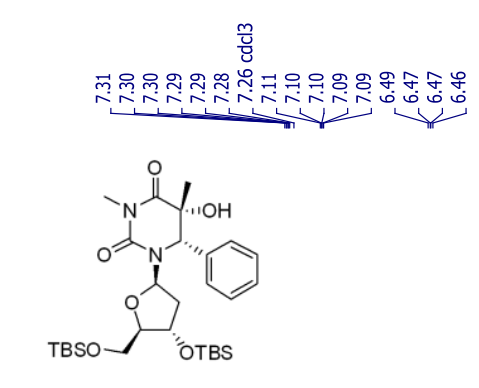

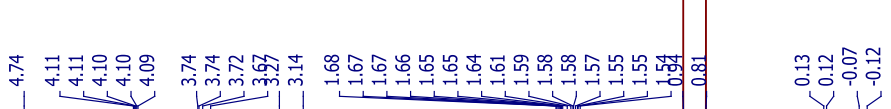

Compound 18
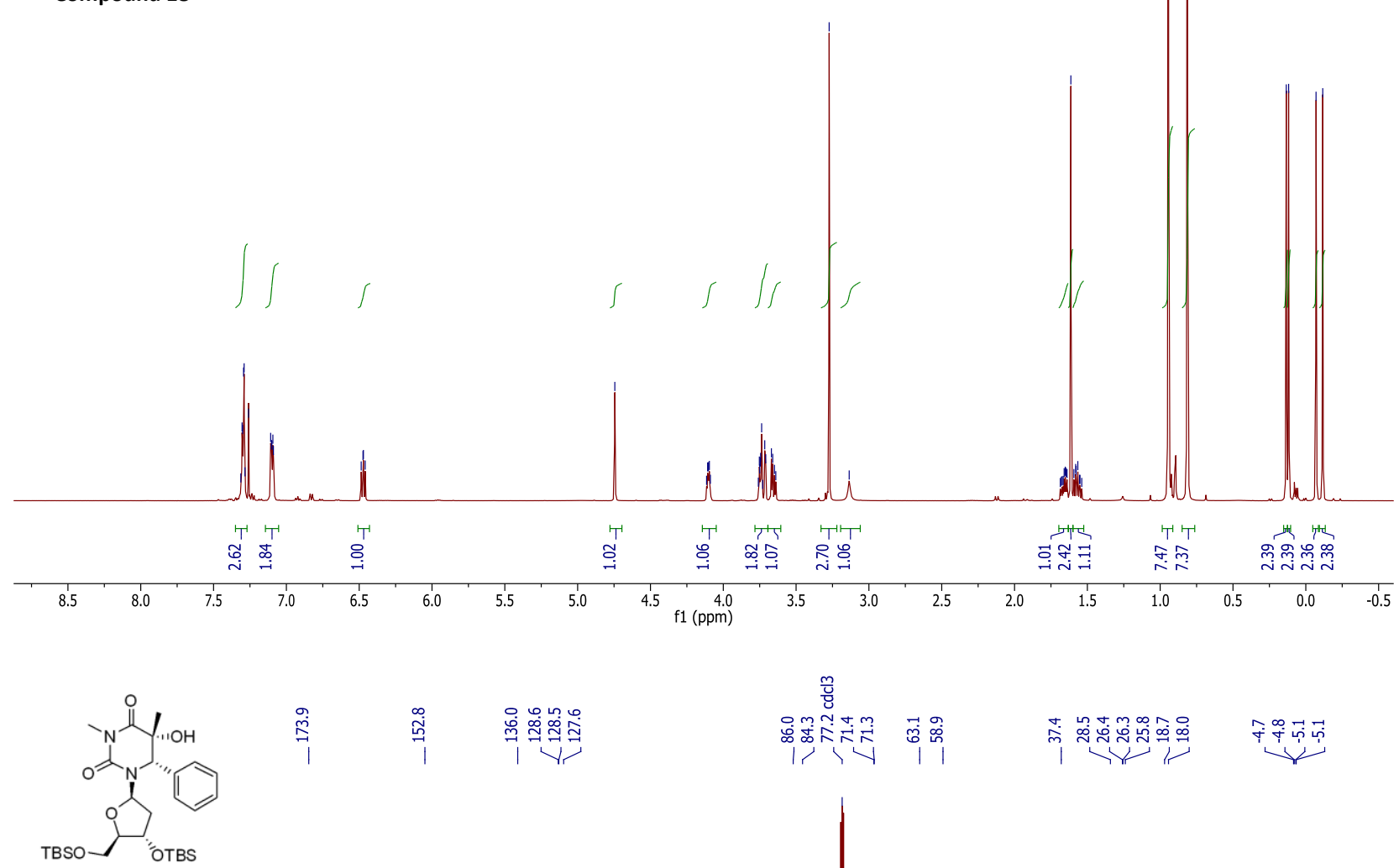

Compound 18

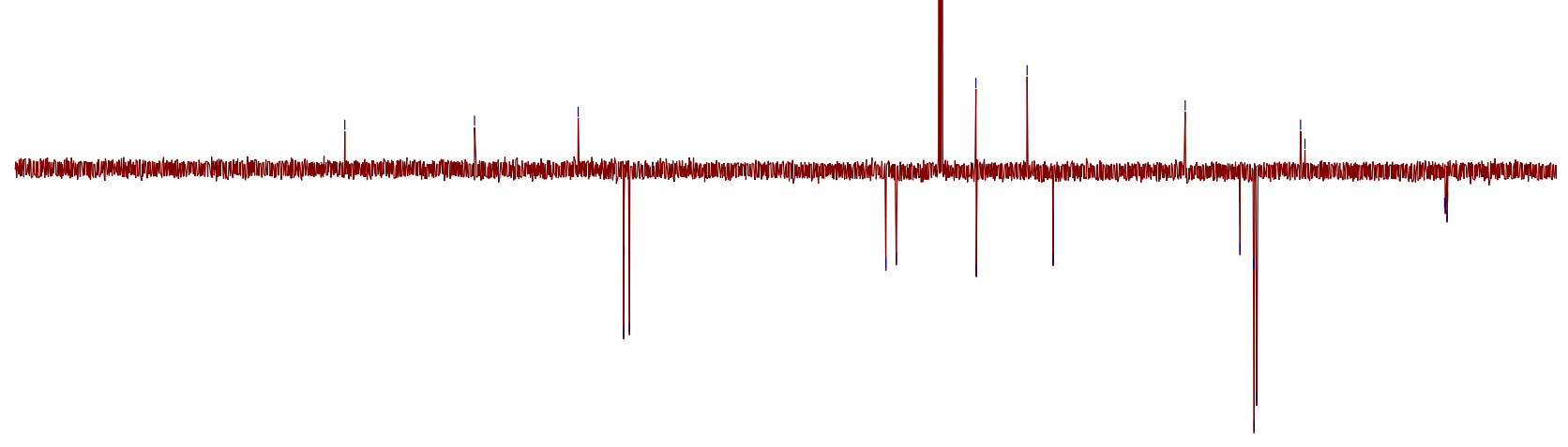

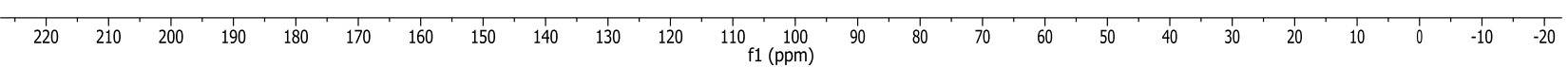




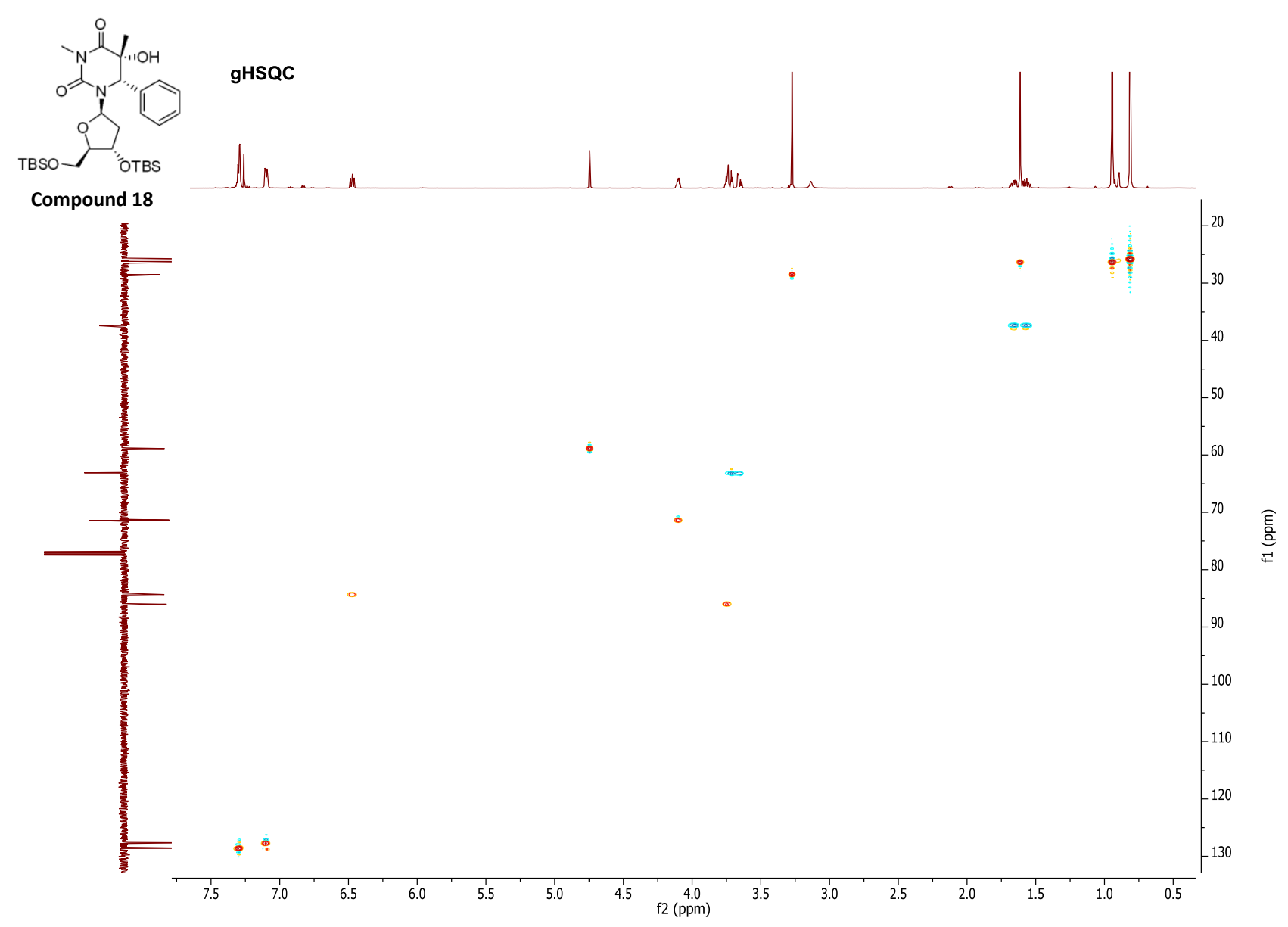



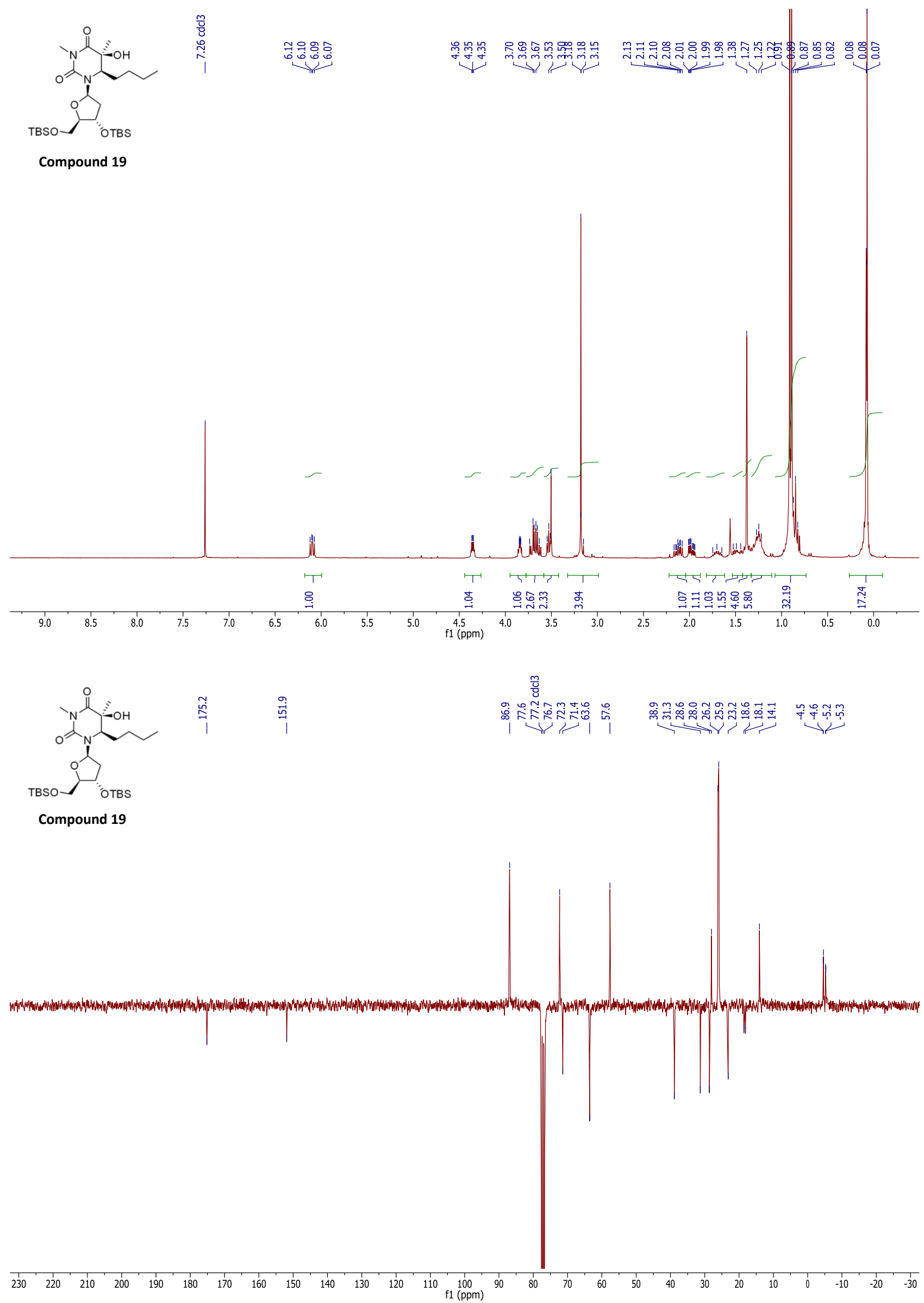

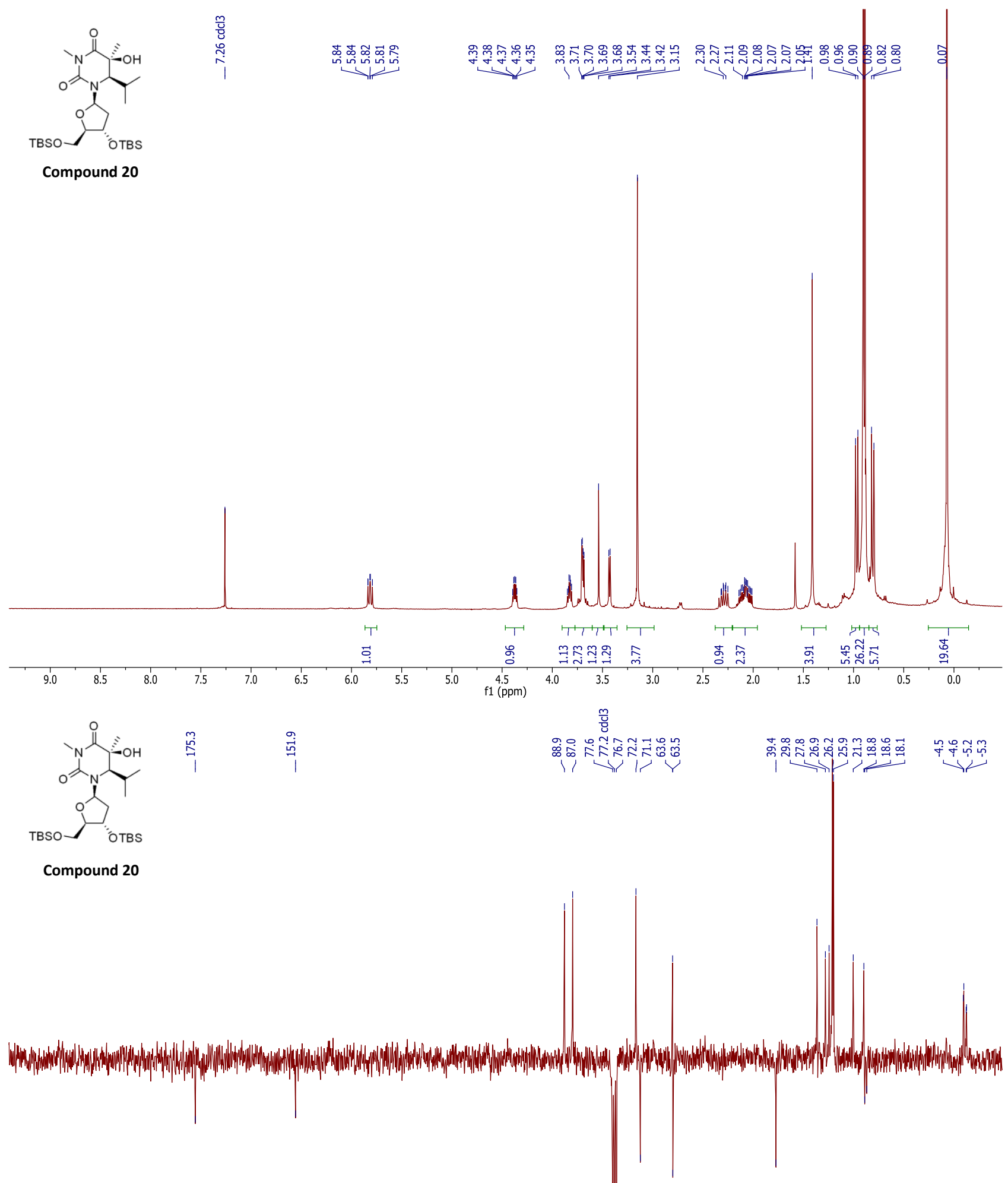

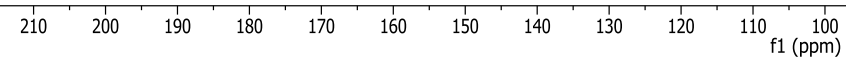



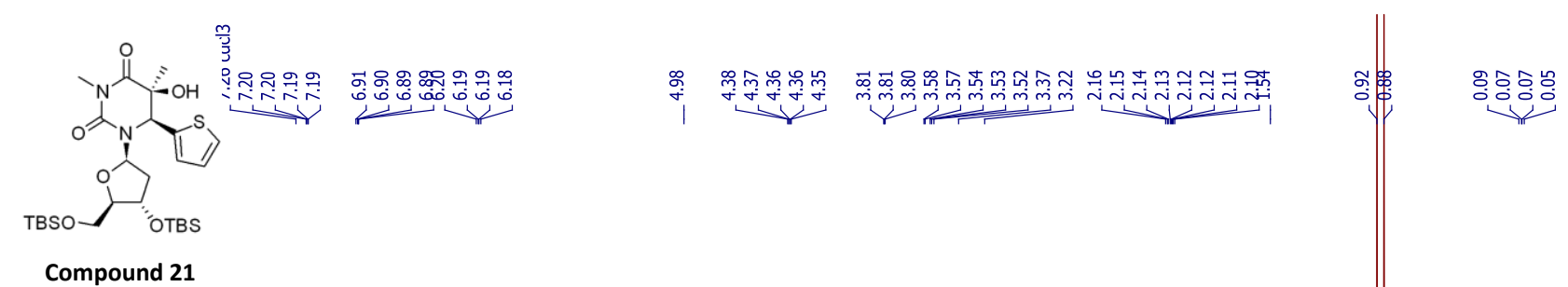

Compound 21
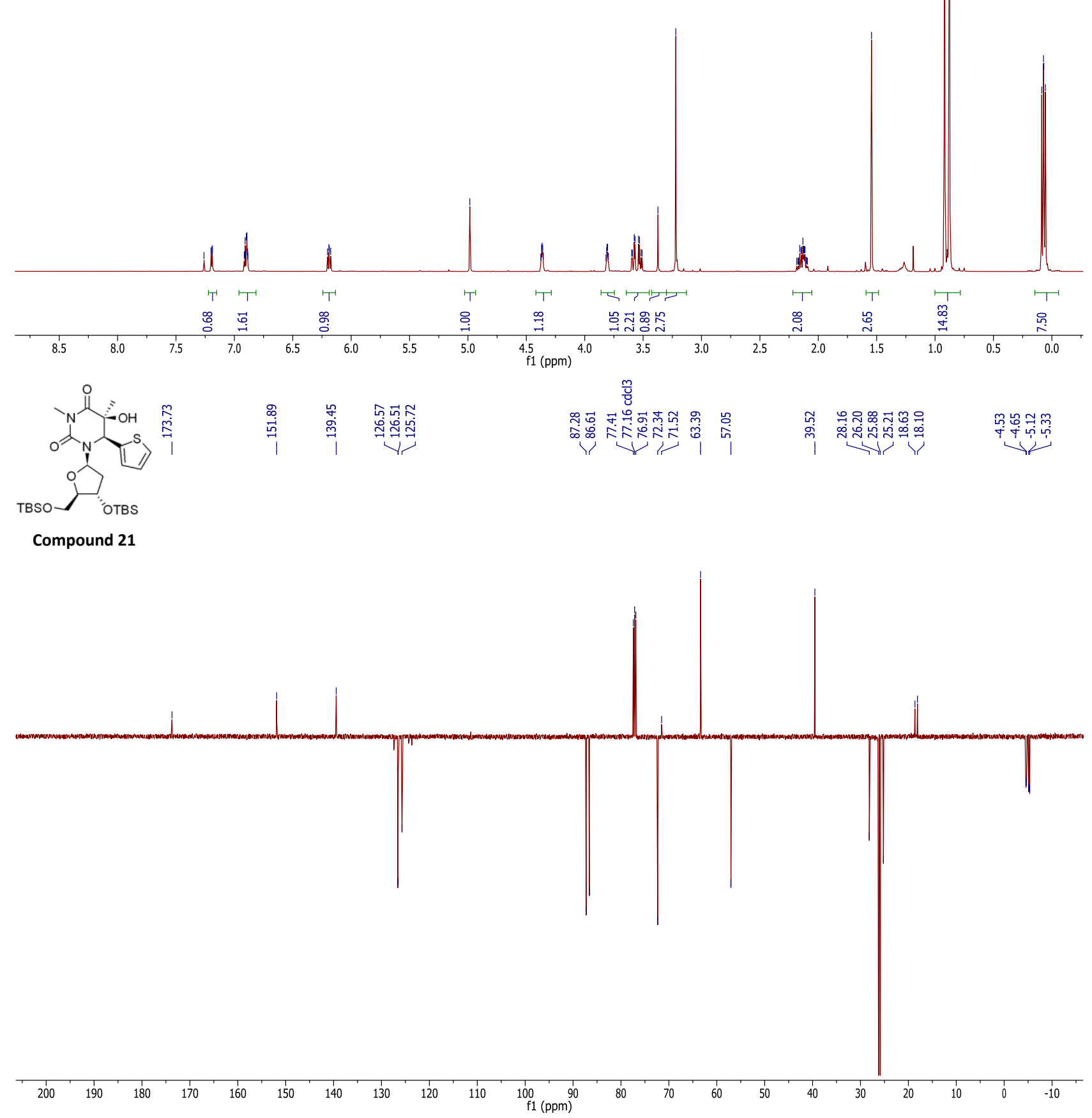

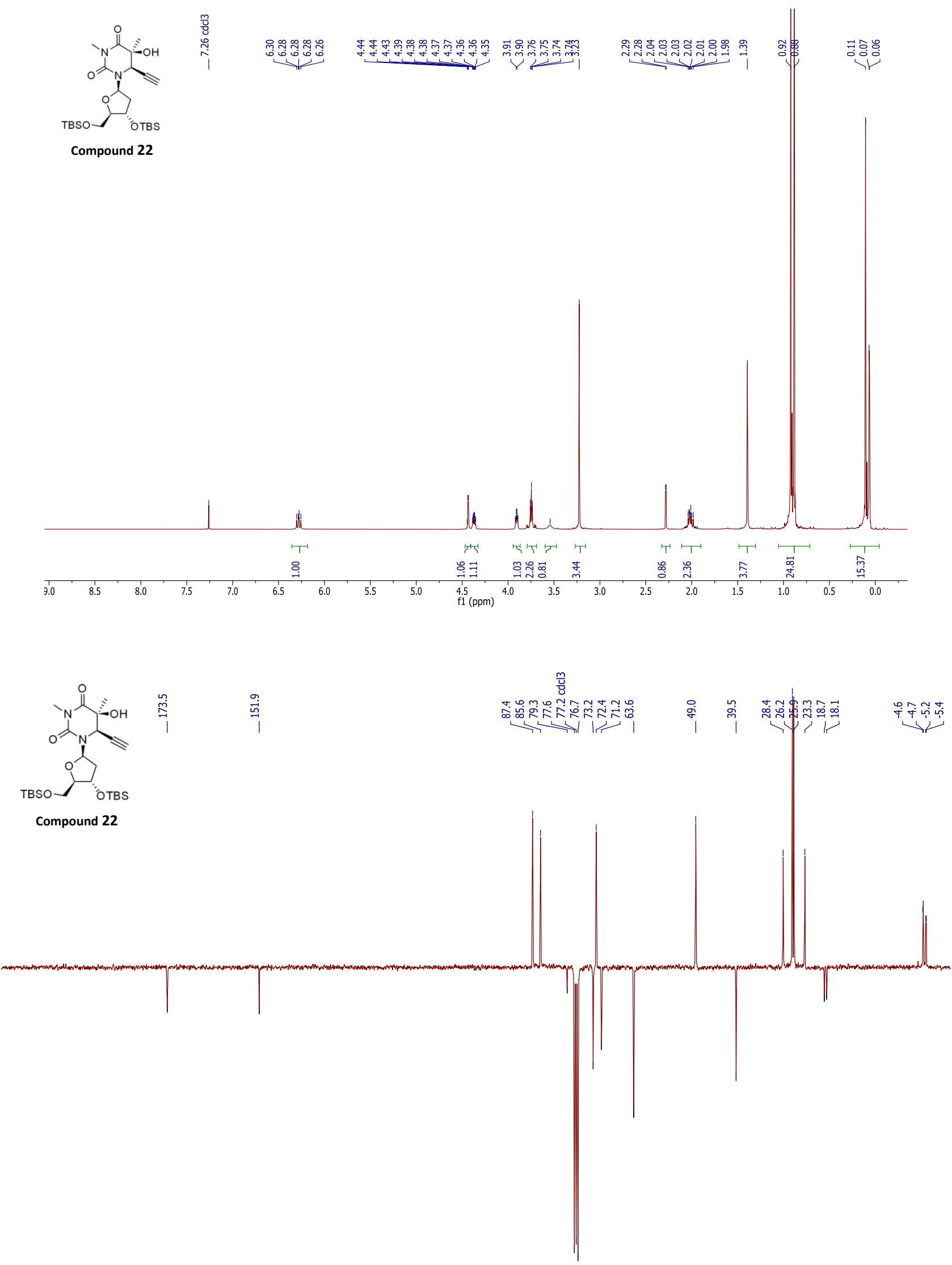

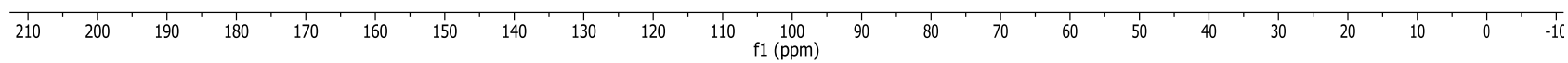



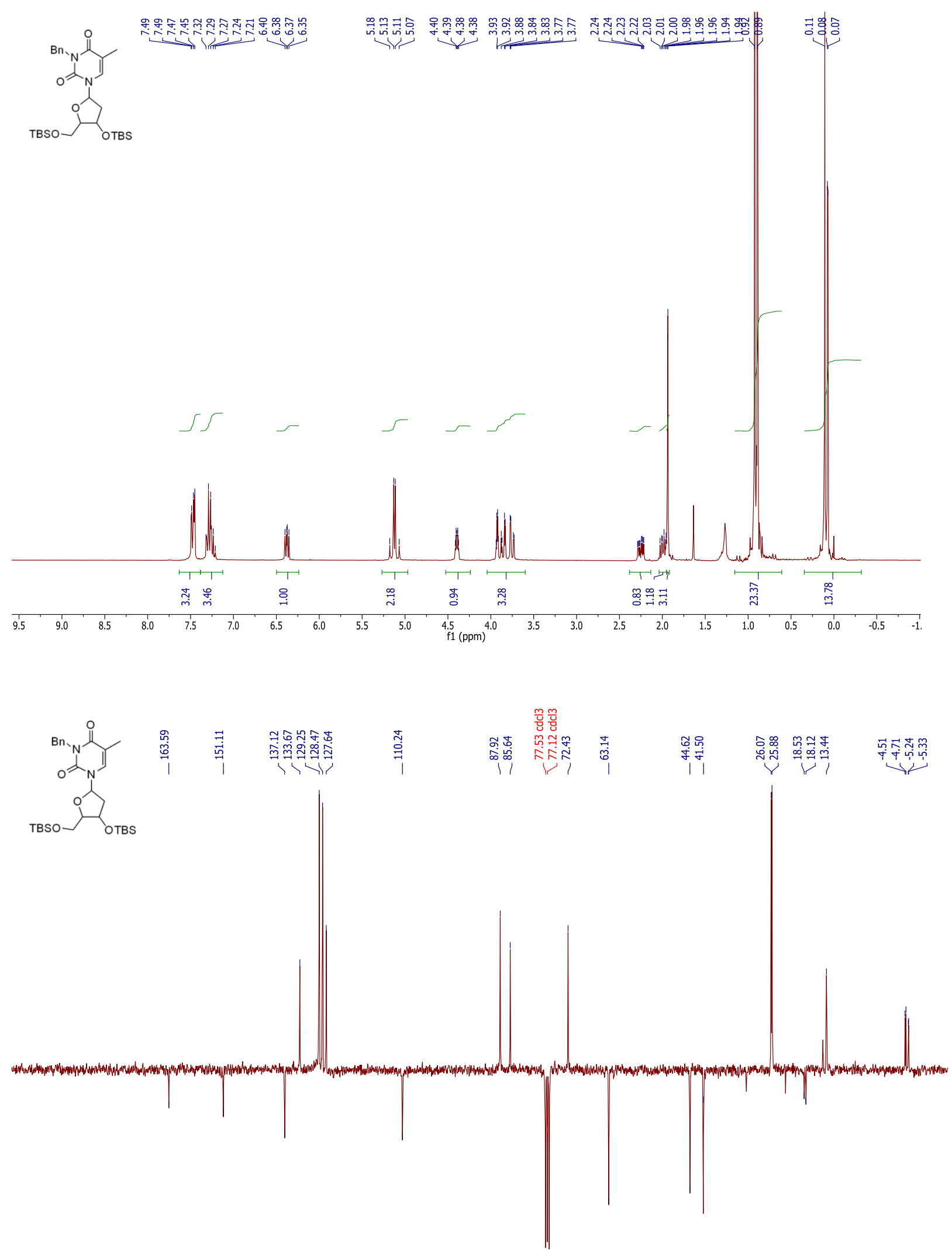

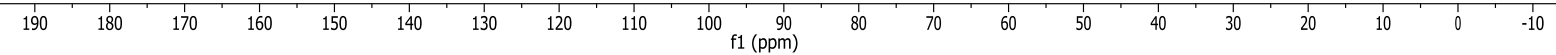




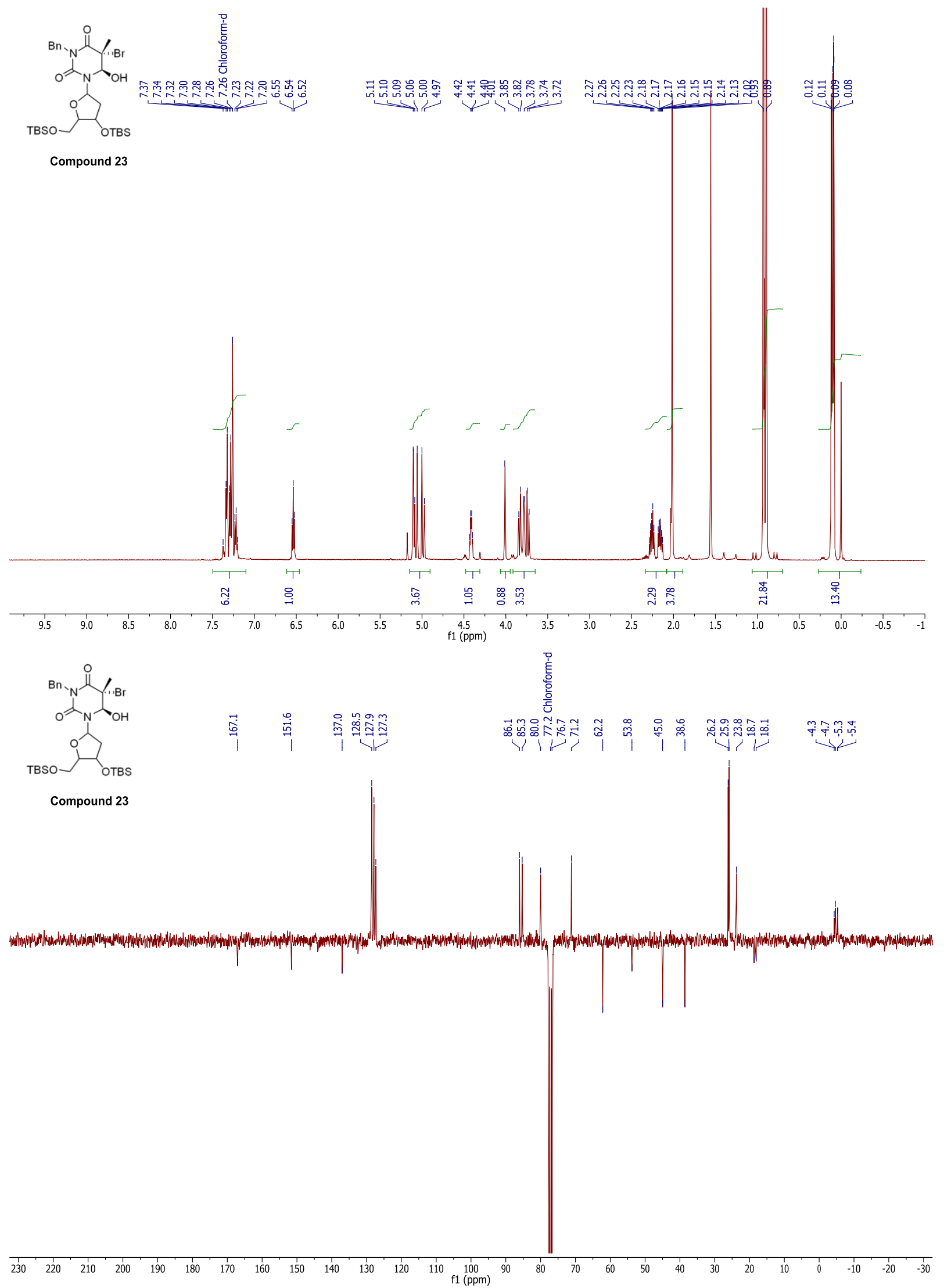



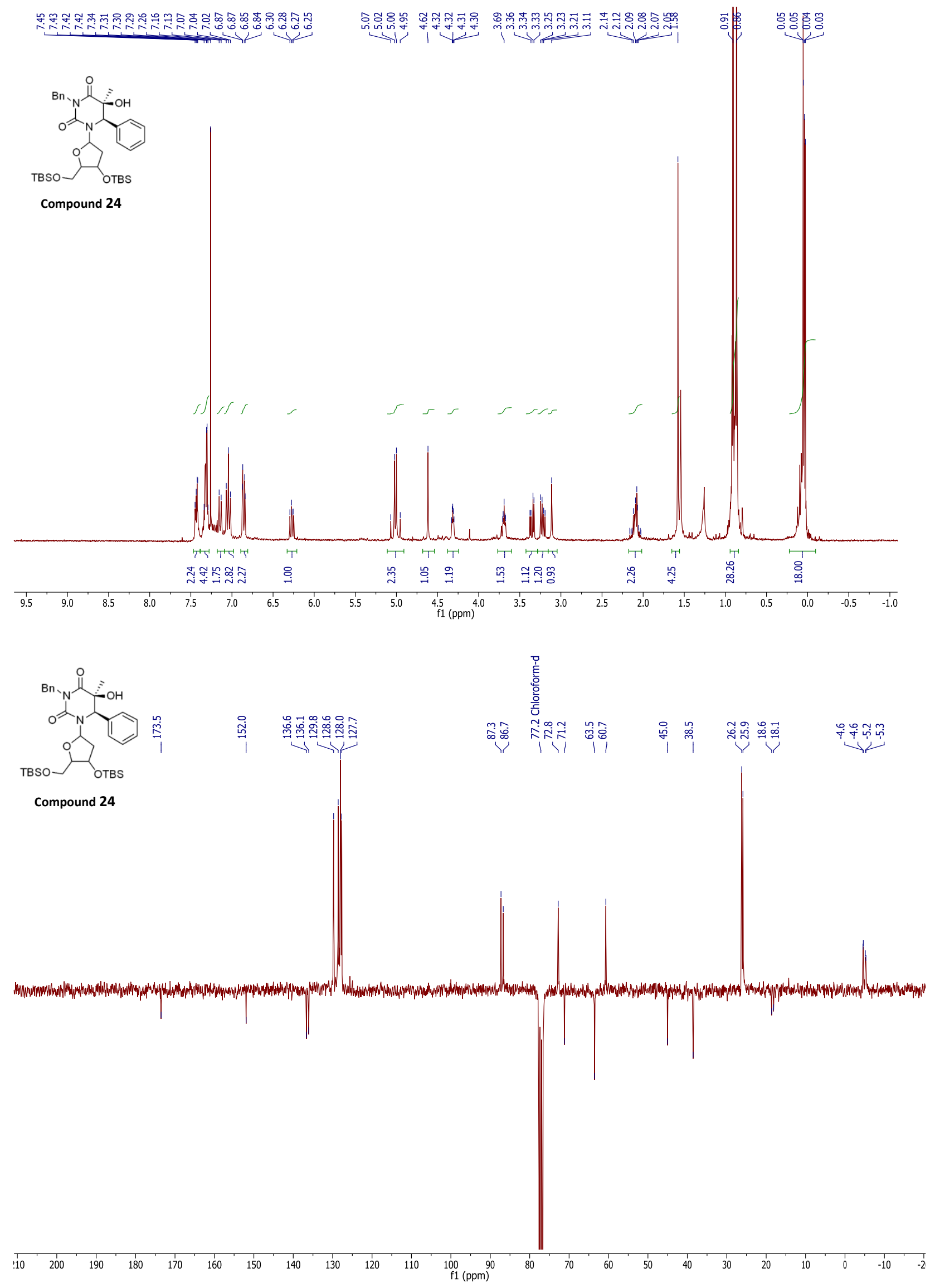


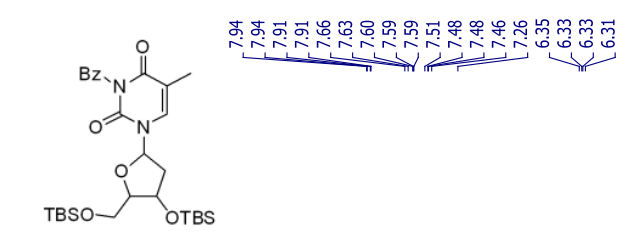

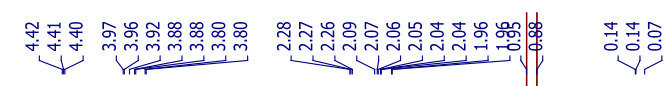
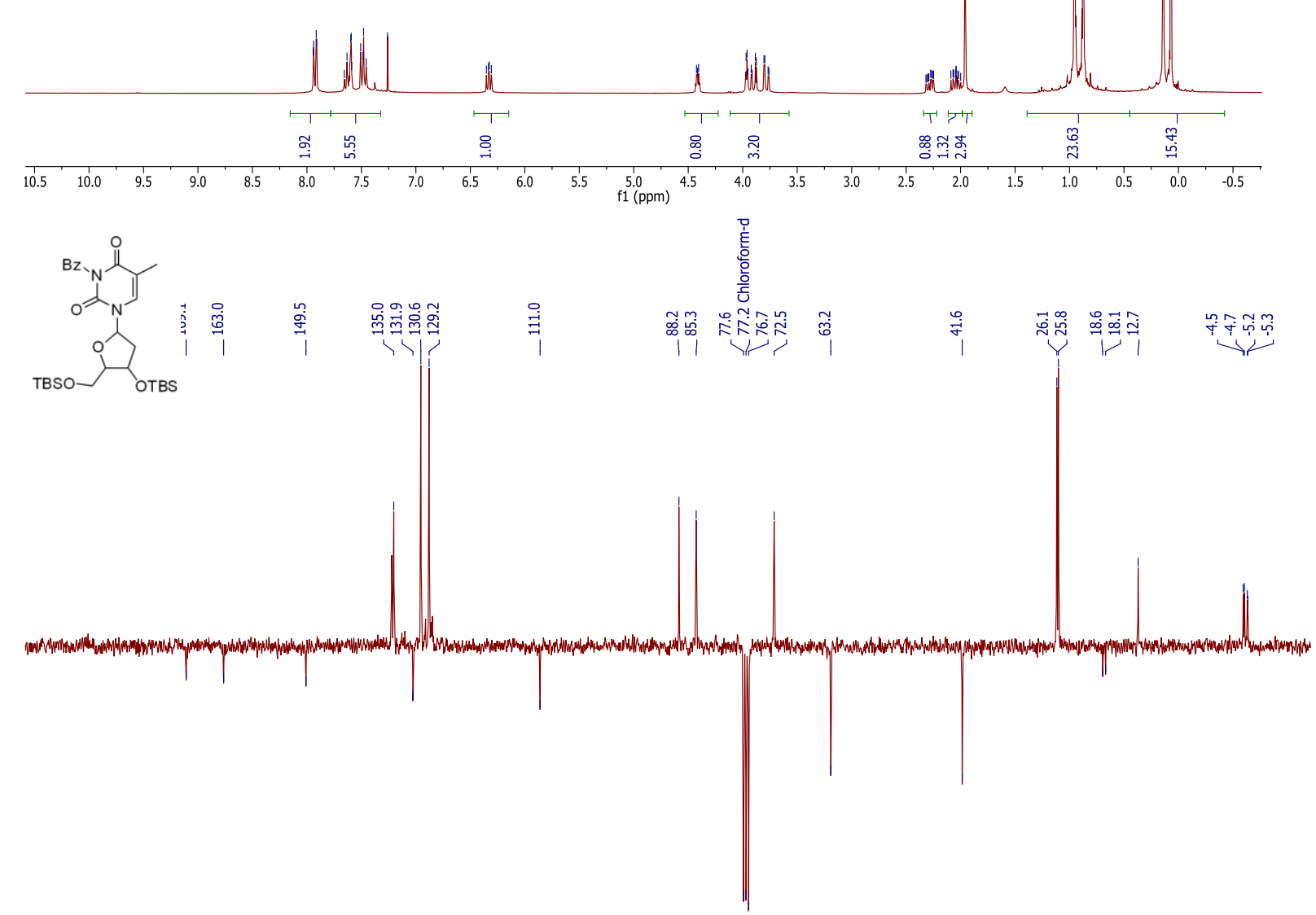

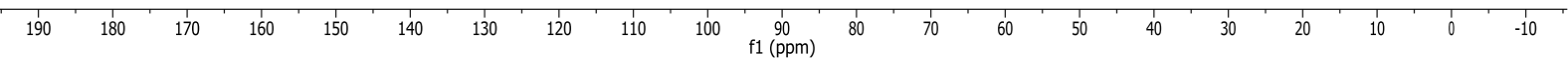




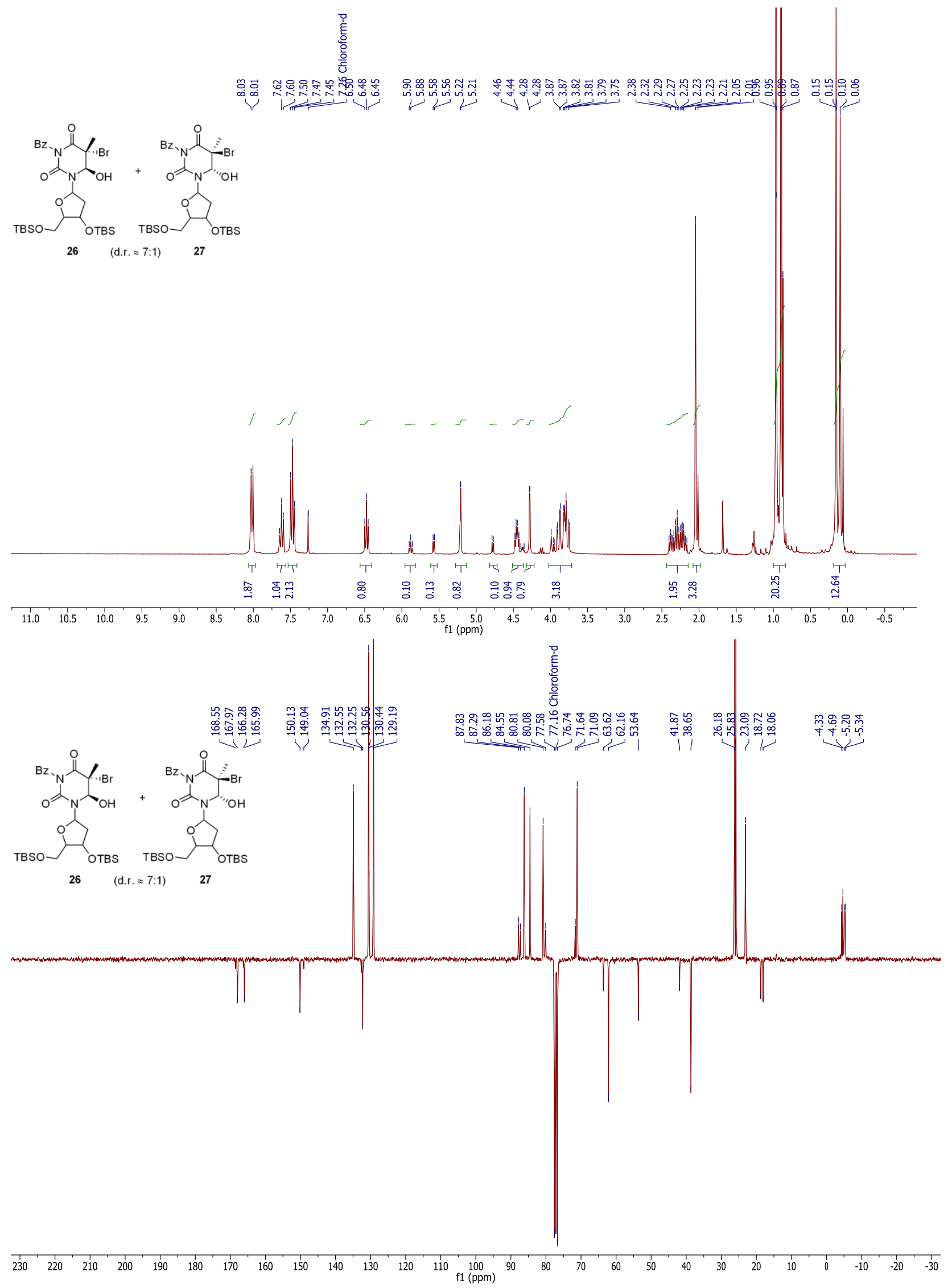



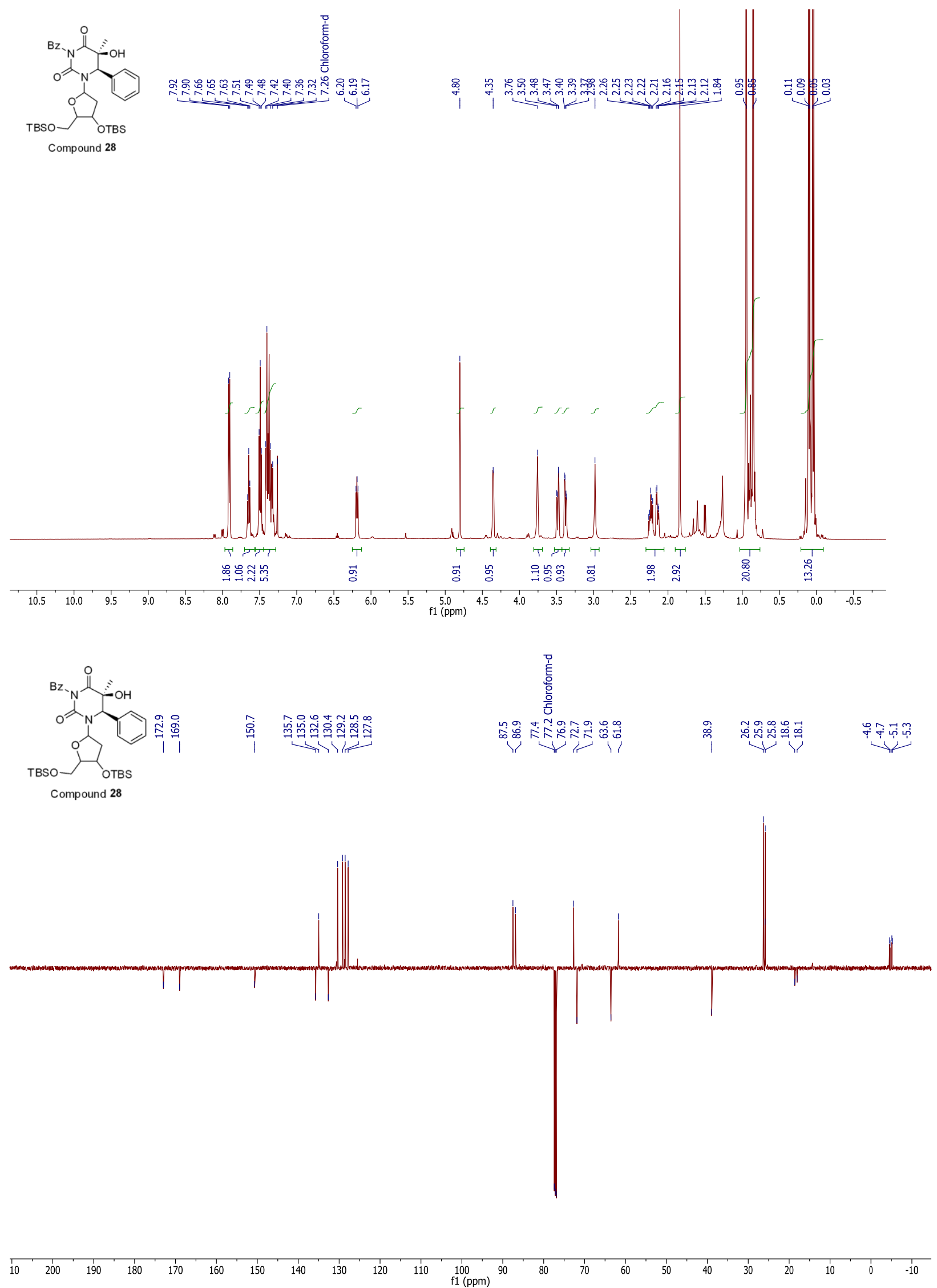


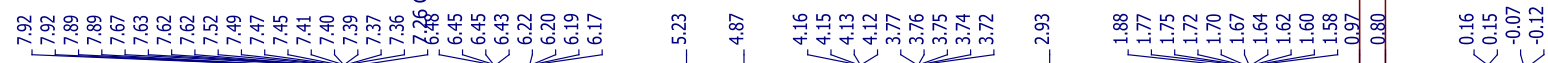
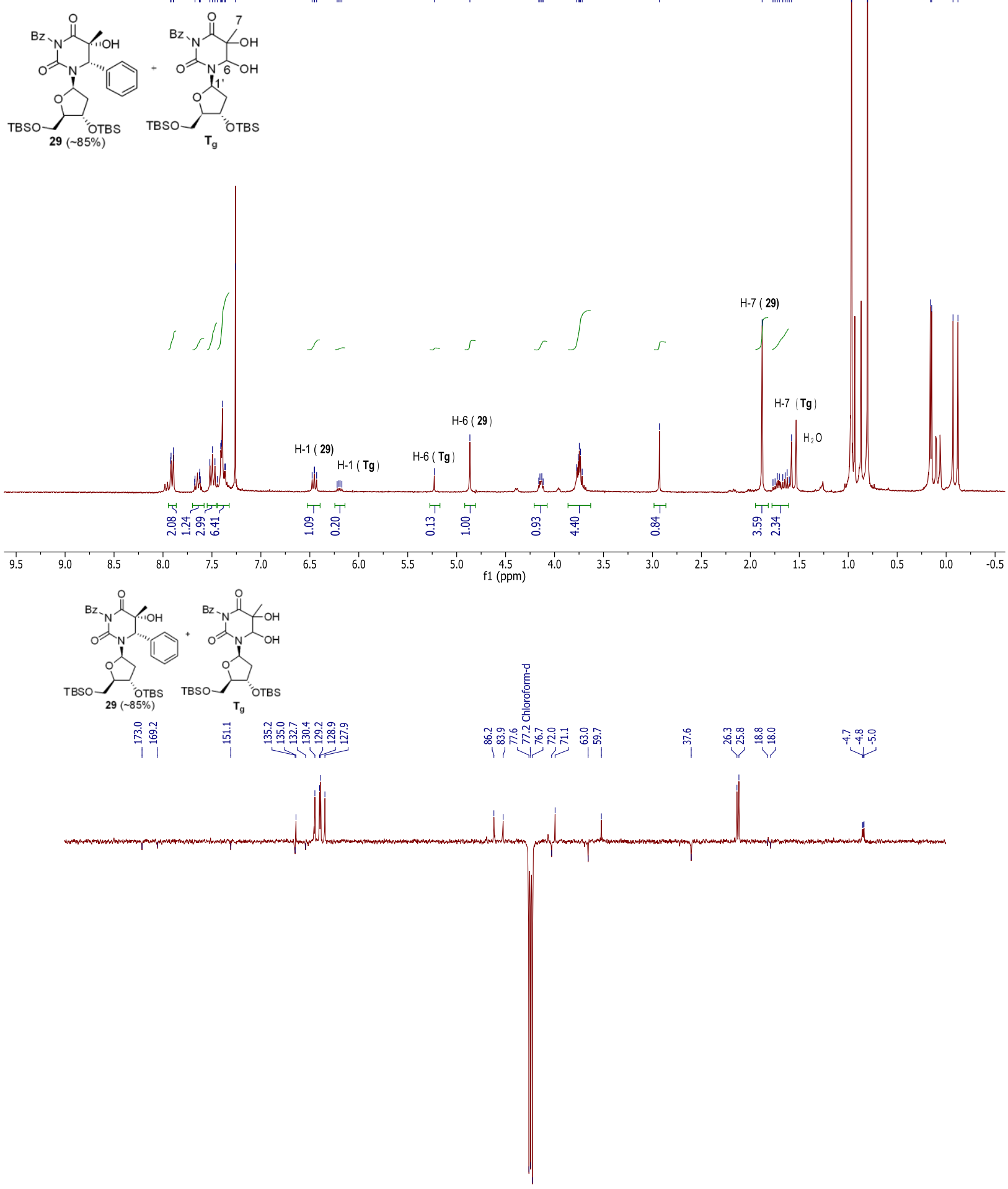

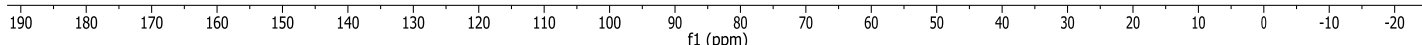



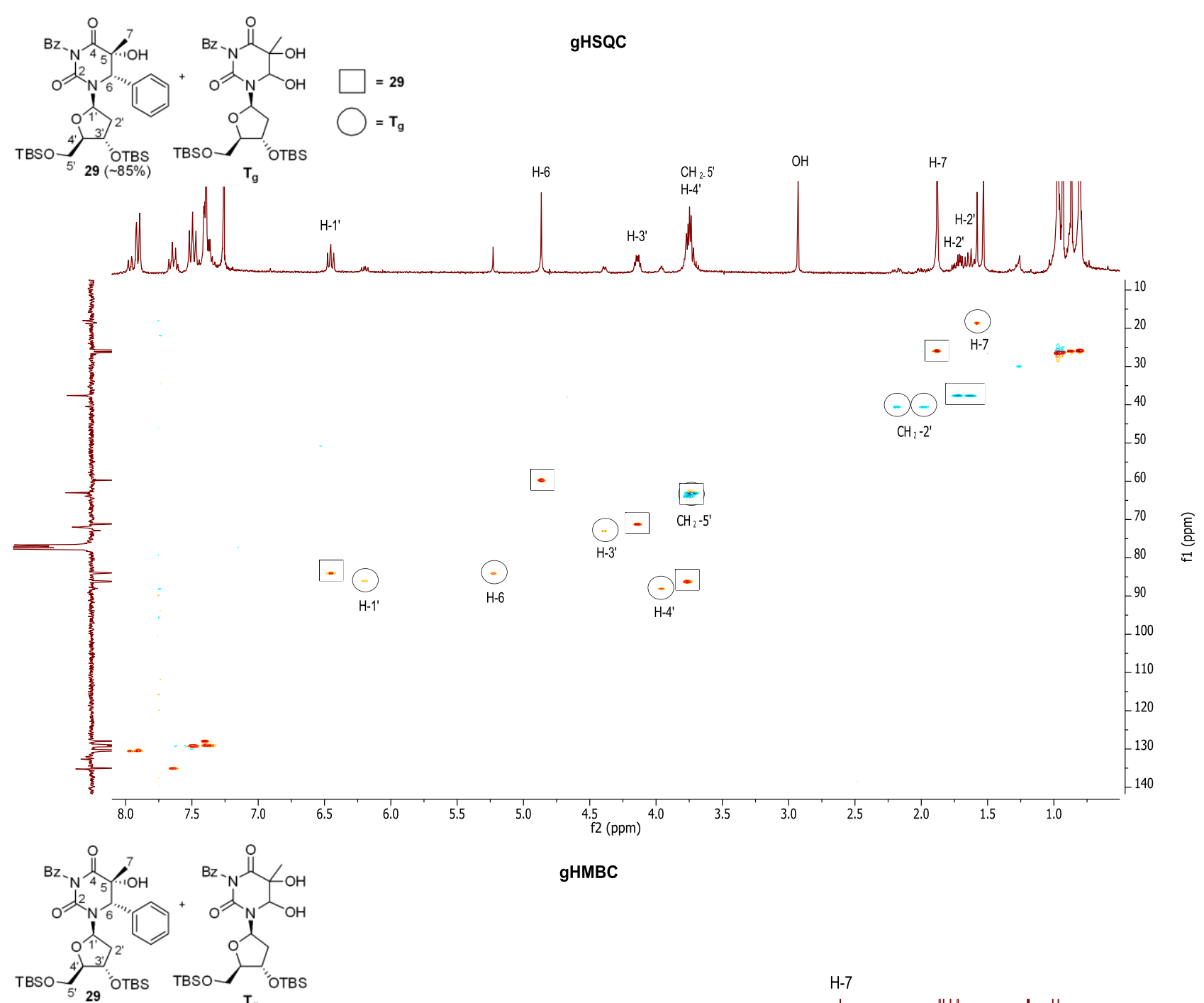

gHMBC

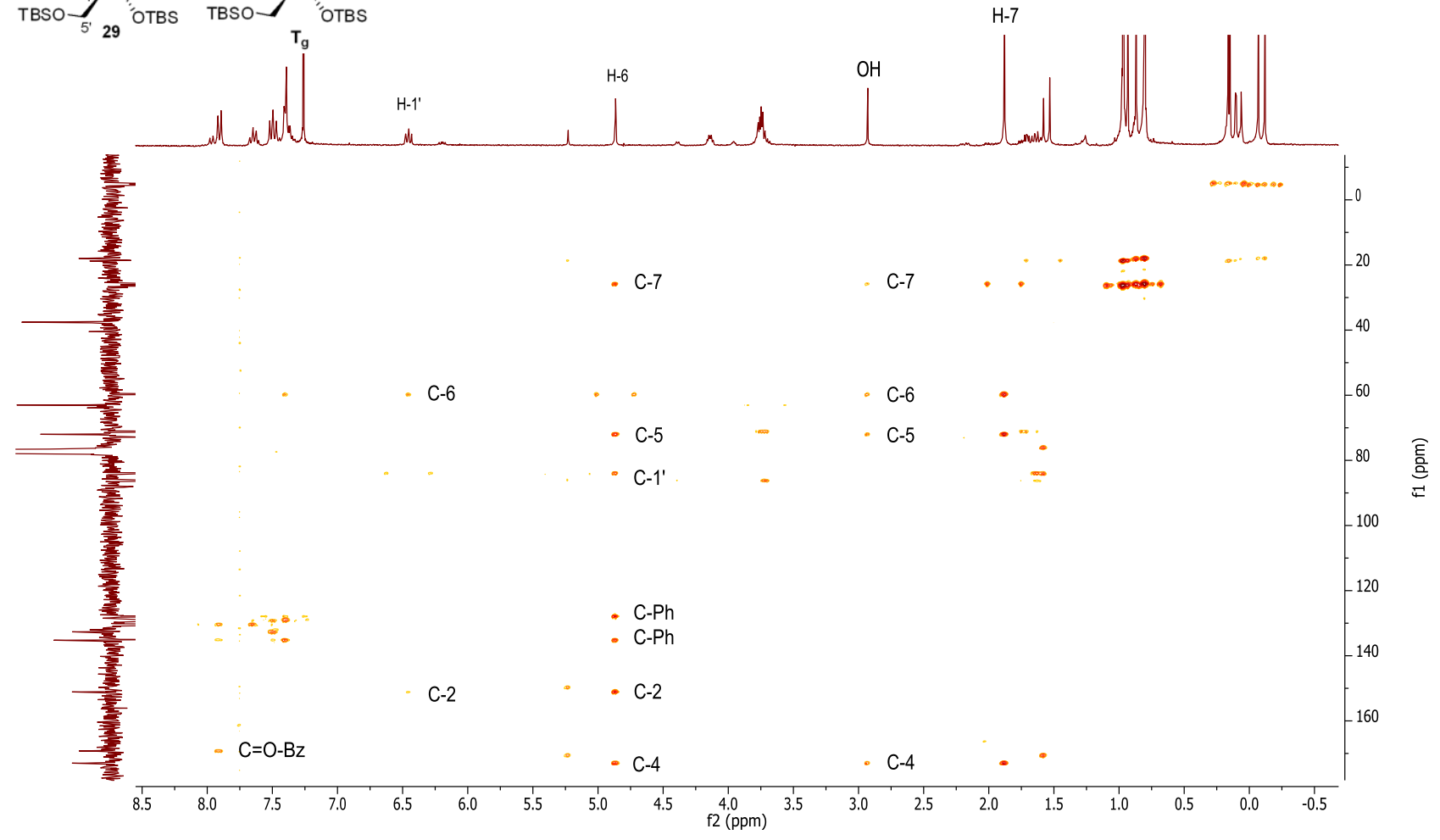



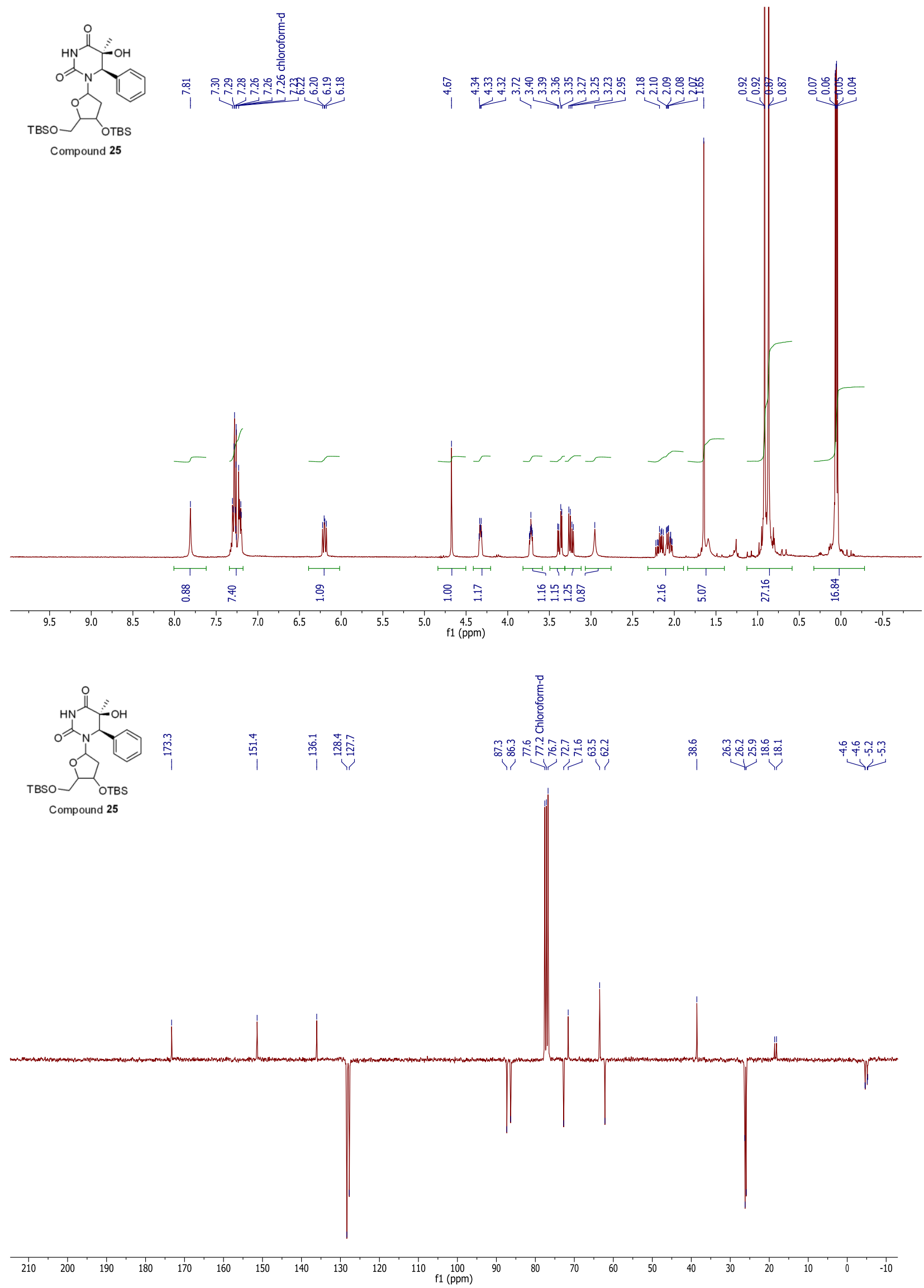


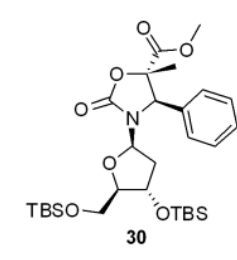

(N)

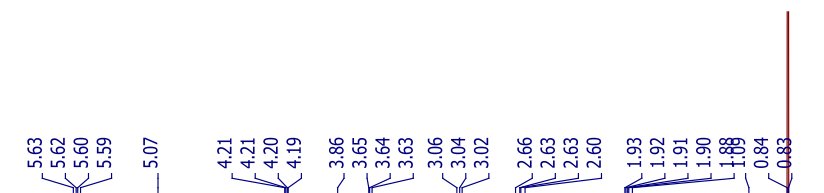

(5)

30
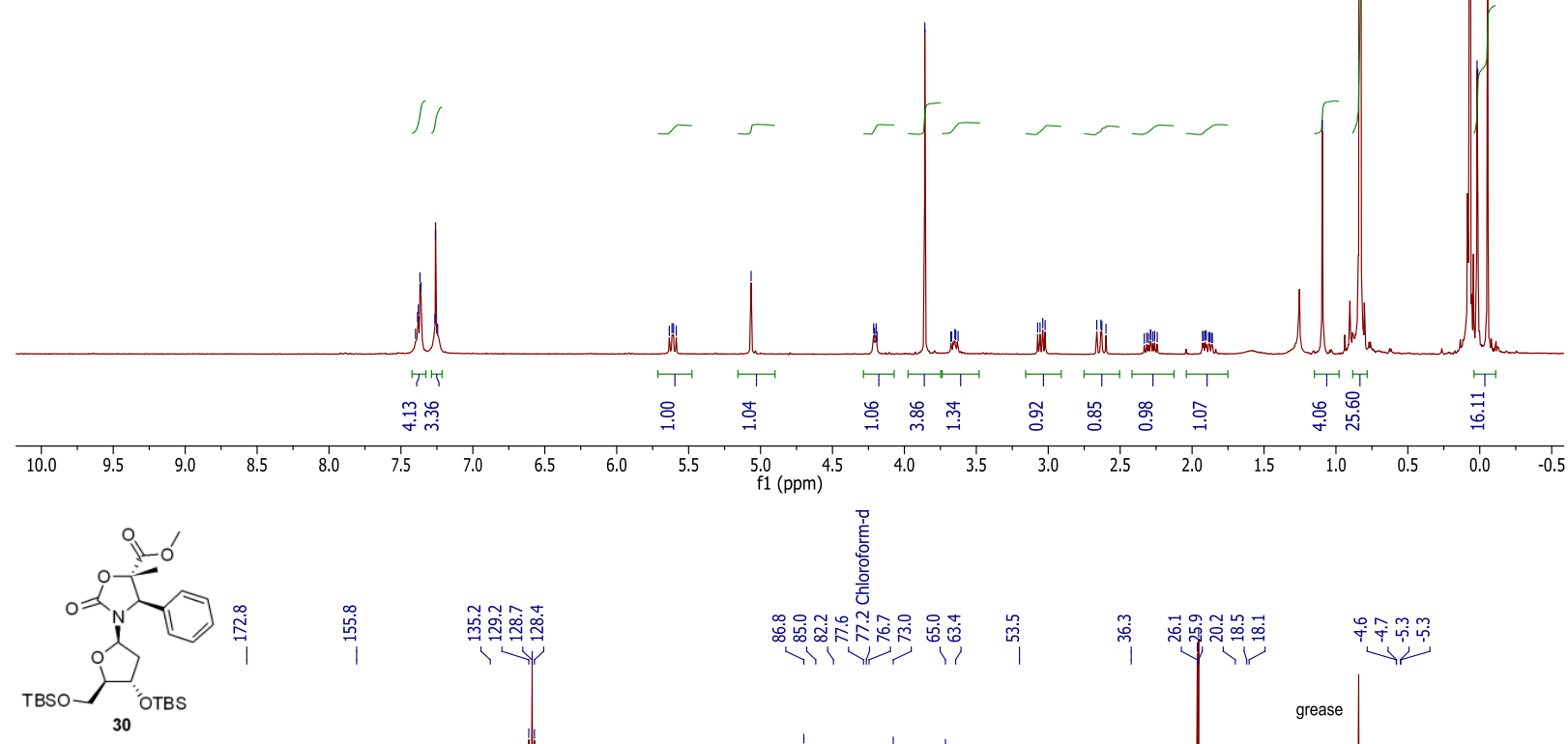

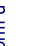




\section{Crystallographic Data of Compounds 3, 9, 18 and 25}

After recrystallization of $\mathbf{3}$ and 9 from $i-\mathrm{PrOH}-\mathrm{H}_{2} \mathrm{O}$ obtained transparent needles were dissolved in acetone, and single crystals suitable for X-ray crystallography were grown by slow evaporation of the solvent. With compound 18 the crystals suitable for X-ray crystallography were grown from n-hexane at- $20{ }^{\circ} \mathrm{C}$. With compound 25 the crystals suitable for X-ray crystallography were grown from $i-\mathrm{PrOH}-\mathrm{H}_{2} \mathrm{O}$ at $25^{\circ} \mathrm{C}$.

The single-crystal X-ray diffraction study of $\mathbf{3}$ and $\mathbf{9}$ were carried out on a Bruker-Nonius KappaCCD diffractometer at $123(2) \mathrm{K}$ using $\operatorname{MoK}_{\alpha}$ radiation $(\lambda=0.71073 \AA$ ), of 18 on an Agilent Supernova diffractometer with Atlas detector at $120(2) \mathrm{K}$ using $\mathrm{CuK}_{\alpha}$ radiation $(\lambda=1.54178 \AA)$ and of $\mathbf{2 5}$ on a Bruker D8 Venture diffractometer with Photo100 detector at $123(2) \mathrm{K}$ using $\mathrm{CuK}_{\alpha}$ radiation $(\lambda=1.54178 \AA)$. Direct Methods (SHELXS-97) (Sheldrick, G. M. Acta Crystallogr A 2008, 64, 112-122) were used for structure solution, and refinement was carried out using SHELXL-97 (3, 9) (Sheldrick, G. M. Acta Crystallogr A 2008, 64, 112-122) and SHELXL-2013/2014 (18, 25) (Sheldrick, G. M. Acta Crystallogr C 2015, 71, 3-8) (full-matrix leastsquares refinement on $\left.F^{2}\right){ }^{3} \mathrm{H}$ atoms were localized by difference Fourier synthesis and refined using a riding model $(\mathrm{H}(\mathrm{O})$ free). A semi-empirical absorption correction was applied for $\mathbf{1 8}$ and 25. The absolute configuration of 18 and $\mathbf{2 5}$ were determined by refinement of the Parsons-Flack parameter (Parsons and Flack (2004), Acta Cryst. A60, s61). For 25 an extinction correction was applied.

3: colorless crystals, $\mathrm{C}_{9} \mathrm{H}_{16} \mathrm{~N}_{2} \mathrm{O}_{3}, M=200.24$, crystal size $0.32 \times 0.16 \times 0.08 \mathrm{~mm}$, triclinic, space group P-1 (No. 2): $a=5.799(1) \AA, b=8.020(1) \AA, c=11.532(1) \AA, \alpha=84.05(1)^{\circ}, \beta=77.38(1)^{\circ}, \gamma=70.70(1)^{\circ}, V=$ $493.47(11) \AA^{3}, Z=2, \rho($ calcd $)=1.347 \mathrm{Mg} \mathrm{m}^{-3}, F(000)=216, \mu=0.101 \mathrm{~mm}^{-1}, 11687$ reflections $\left(2 \theta_{\max }=55^{\circ}\right)$, 2266 unique $\left[\mathrm{R}_{\text {int }}=0.027\right], 132$ parameters, 1 restraint, $\mathrm{R} 1$ (for $\left.1981 I>2 \sigma(I)\right)=0.037, \mathrm{wR} 2$ (all data) $=0.102$, GooF $=1.05$, largest diff. peak and hole 0.319 and -0.263 e $\AA^{-3}$.

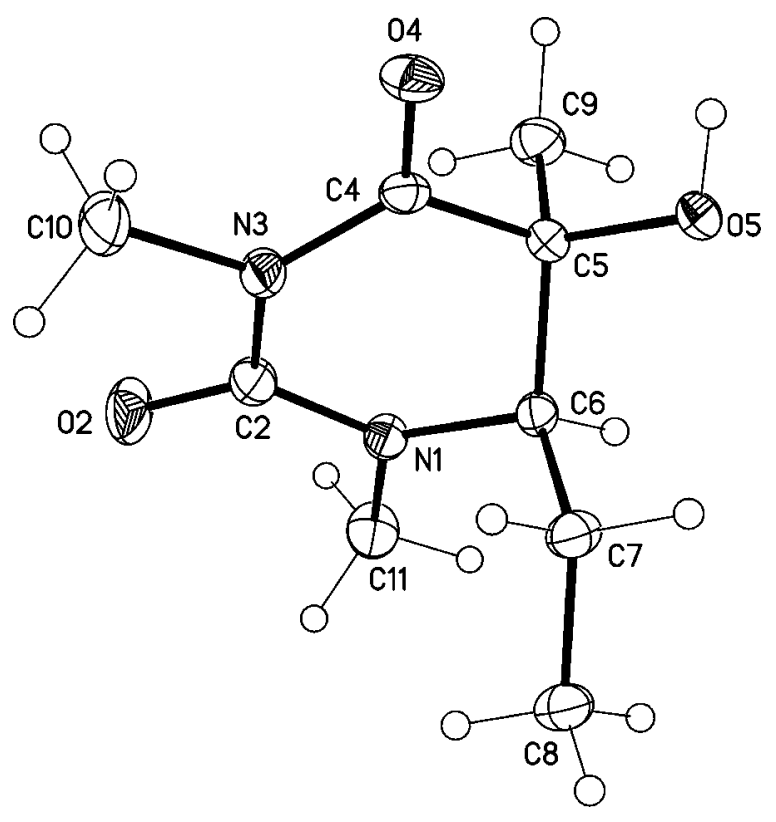

Figure SI1. Molecular structure of $\mathbf{3}$ (displacement parameters are drawn at 50\% probability level). 
9: colorless crystals, $\mathrm{C}_{13} \mathrm{H}_{16} \mathrm{~N}_{2} \mathrm{O}_{3}, M=248.28$, crystal size $0.40 \times 0.5 \times 0.15 \mathrm{~mm}$, triclinic, space group P-1 (No. 2): $a=9.4062(4) \AA, b=11.7763(15) \AA, c=11.8030(14) \AA, \alpha=74.743(10)^{\circ}, \beta=79.948(6)^{\circ}, \gamma=$ $80.083(6)^{\circ}, V=1231.0(2) \AA^{3}, Z=4, \rho($ calcd $)=1.340 \mathrm{Mg} \mathrm{m}^{-3}, F(000)=528, \mu=0.096 \mathrm{~mm}^{-1}, 48069$ reflections $\left(2 \theta_{\max }=55^{\circ}\right), 5640$ unique $\left[\mathrm{R}_{\text {int }}=0.029\right], 335$ parameters, 21 restraints, R1 (for $\left.4924 I>2 \sigma(I)\right)=0.037$, wR2 $($ all data $)=0.098, \mathrm{GooF}=1.05$, largest diff. peak and hole 0.341 and -0.308 e $\AA^{-3}$.

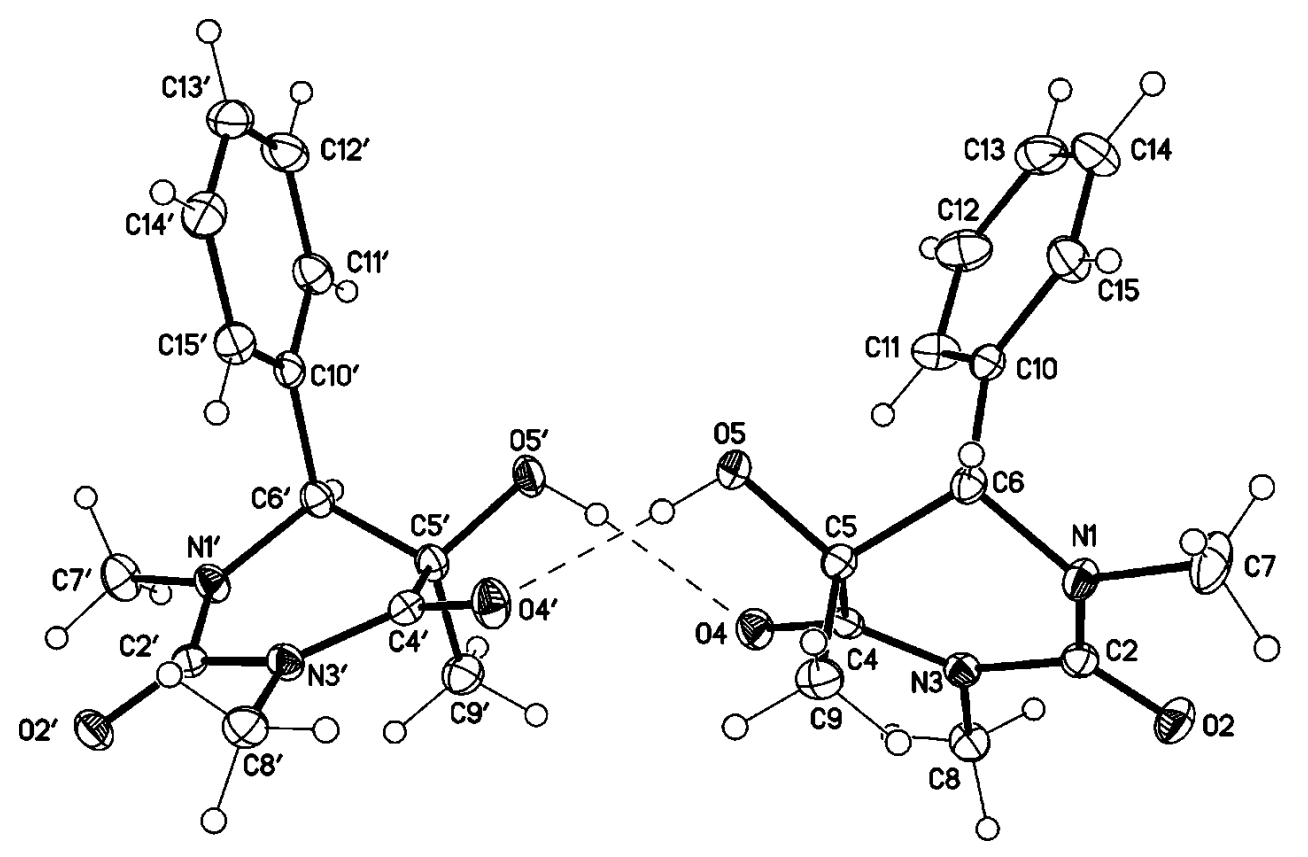

Figure SI2. Molecular structure of 9 (displacement parameters are drawn at 50\% probability level; both crystallographic independent molecules are shown).

18: colorless crystals, $\mathrm{C}_{29} \mathrm{H}_{50} \mathrm{~N}_{2} \mathrm{O}_{6} \mathrm{Si}_{2}, M=578.89$, crystal size $0.30 \times 0.15 \times 0.15 \mathrm{~mm}$, orthorhombic, space group P2 $2_{2} 2_{1}$ (No. 19): $a=8.21605(5) \AA, b=15.48832(9) \AA, c=26.12024(14) \AA, V=3323.87(3) \AA^{3}, Z=4$, $\rho($ calcd $)=1.157 \mathrm{Mg} \mathrm{m}^{-3}, F(000)=1256, \mu=1.294 \mathrm{~mm}^{-1}, 17714$ reflections $\left(2 \theta_{\max }=154^{\circ}\right), 6955$ unique $\left[\mathrm{R}_{\text {int }}\right.$ $=0.021], 357$ parameters, 1 restraint, R1 (for $6867 I>2 \sigma(I))=0.027, \mathrm{wR} 2($ all data $)=0.069, \mathrm{GooF}=1.06$, largest diff. peak and hole 0.222 and $-0.170 \mathrm{e} \AA^{-3}$, absolute structure parameter $\mathrm{x}=0.002(6)$. 


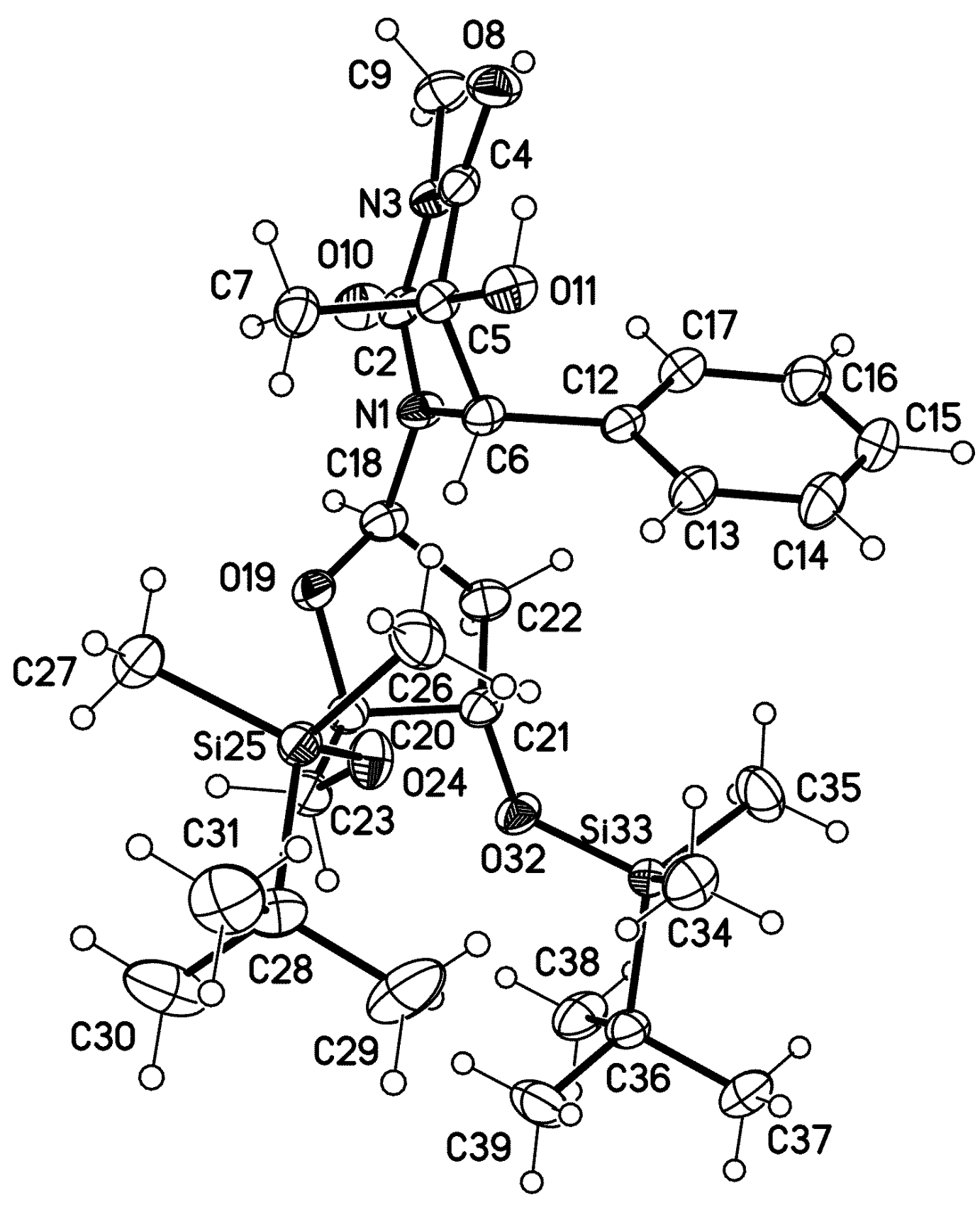

Figure SI3. Molecular structure of $\mathbf{1 8}$ (displacement parameters are drawn at 50\% probability level).

25: colorless crystals, $\mathrm{C}_{28} \mathrm{H}_{48} \mathrm{~N}_{2} \mathrm{O}_{6} \mathrm{Si}_{2}, M=564.86$, crystal size $0.18 \times 0.04 \times 0.02 \mathrm{~mm}$, orthorhombic, space group P2 $22_{1} 2_{1}$ (No. 19): $a=6.3869(2) \AA, b=23.9885(7) \AA, c=41.8755(14) \AA, V=6415.7(3) \AA^{3}, Z=8$, $\rho($ calcd $)=1.170 \mathrm{Mg} \mathrm{m}^{-3}, F(000)=2448, \mu=1.329 \mathrm{~mm}^{-1}, 38957$ reflections $\left(2 \theta_{\max }=145.2^{\circ}\right), 12462$ unique $\left[\mathrm{R}_{\text {int }}=0.093\right.$ ], 698 parameters, 4 restraint, R1 (for $\left.9129 I>2 \sigma(I)\right)=0.056$, wR2 (all data) $=0.126$, GooF $=$ 1.03 , largest diff. peak and hole 0.342 and $-0.269 \mathrm{e} \AA^{-3}$, absolute structure parameter $\mathrm{x}=0.07(2)$. 


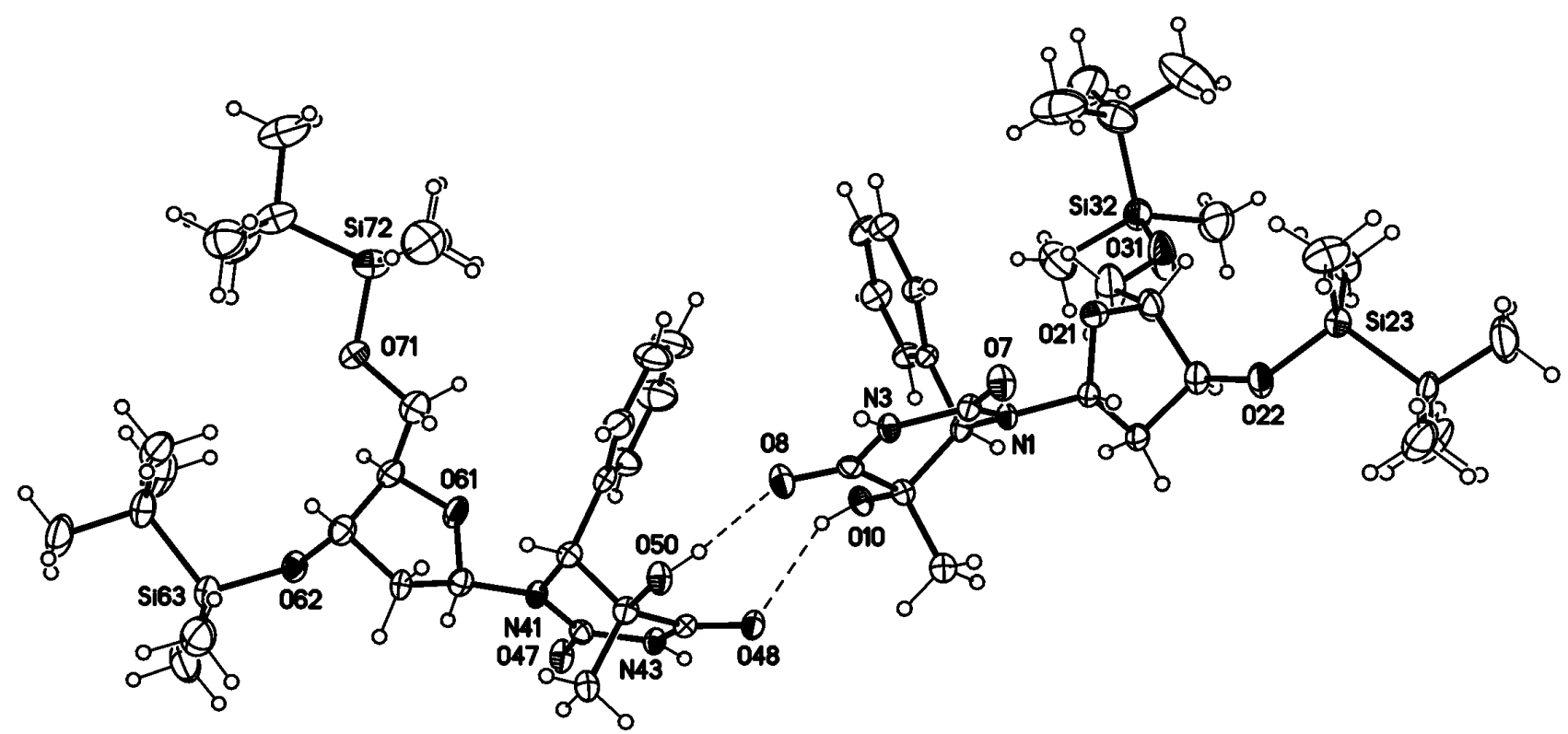

Figure SI4. Molecular structure of $\mathbf{2 5}$ (displacement parameters are drawn at 50\% probability level).

CCDC-934918 (3), CCDC-934919 (9), CCDC-1004127 (18) and CCDC-1448807 (25) contain the supplementary crystallographic data for this paper. These data can be obtained free of charge from The Cambridge Crystallographic Data Centre via www.ccdc.cam.ac.uk/data_request/cif. 
Cartesian Coordinates For All B3LYP / 631G(D) Optimized Structures.

$$
C I S-1\left(\mathrm{C}_{7} \mathrm{H}_{11} \mathrm{BrN}_{2} \mathrm{O}_{3}\right)
$$

$\begin{array}{lrrr}\mathrm{N} & 1.502469 & -1.006702 & -0.135004 \\ \mathrm{C} & 1.887952 & 0.317678 & 0.202559 \\ \mathrm{~N} & 0.996234 & 1.304315 & -0.138076 \\ \mathrm{C} & -0.106661 & 1.021123 & -1.056939 \\ \mathrm{C} & -0.806495 & -0.283857 & -0.674433 \\ \mathrm{C} & 0.227619 & -1.402331 & -0.508834 \\ \mathrm{O} & -0.055674 & -2.569827 & -0.710444 \\ \mathrm{O} & 2.960392 & 0.527864 & 0.748598 \\ \mathrm{C} & 2.490055 & -2.065470 & 0.112685 \\ \mathrm{C} & 1.388645 & 2.691516 & 0.084816 \\ \mathrm{H} & 3.484807 & -1.636335 & 0.015031 \\ \mathrm{H} & 2.372949 & -2.472377 & 1.122198 \\ \mathrm{H} & 2.327789 & -2.861765 & -0.612644 \\ \mathrm{H} & 2.183441 & 2.694341 & 0.828532 \\ \mathrm{H} & 1.774913 & 3.158704 & -0.833856 \\ \mathrm{H} & 0.533271 & 3.262332 & 0.449725 \\ \mathrm{C} & -1.888136 & -0.676011 & -1.669535 \\ \mathrm{H} & -2.663825 & 0.090349 & -1.706462 \\ \mathrm{H} & -2.319823 & -1.637344 & -1.391223 \\ \mathrm{H} & -1.443003 & -0.783240 & -2.667279 \\ \mathrm{H} & 0.299956 & 0.858958 & -2.074580 \\ \mathrm{Br} & -1.654182 & -0.071268 & 1.138439 \\ \mathrm{O} & -1.038512 & 2.066247 & -1.080693 \\ \mathrm{H} & -0.730827 & 2.744201 & -1.700652\end{array}$

\begin{tabular}{lrrr}
\multicolumn{4}{c}{ TRANS-1 $\left(\mathrm{C}_{7} \mathrm{H}_{11} \mathrm{BrN}_{2} \mathrm{O}_{3}\right)$} \\
$\mathrm{N}$ & -1.205957 & -1.196292 & -0.105583 \\
$\mathrm{C}$ & -1.825862 & 0.031628 & -0.440218 \\
$\mathrm{~N}$ & -1.170266 & 1.180609 & -0.047749 \\
$\mathrm{C}$ & -0.120863 & 1.149332 & 0.925809 \\
$\mathrm{C}$ & 0.828616 & -0.035373 & 0.683673 \\
$\mathrm{C}$ & 0.051972 & -1.334777 & 0.452025 \\
$\mathrm{O}$ & 0.528167 & -2.418946 & 0.738406 \\
$\mathrm{O}$ & -2.878356 & 0.042567 & -1.056532 \\
$\mathrm{C}$ & -1.945943 & -2.421349 & -0.436881 \\
$\mathrm{C}$ & -1.865586 & 2.453092 & -0.238005 \\
$\mathrm{H}$ & -3.011596 & -2.209957 & -0.379045 \\
$\mathrm{H}$ & -1.702152 & -2.753337 & -1.451117 \\
$\mathrm{H}$ & -1.656525 & -3.194081 & 0.273402 \\
$\mathrm{H}$ & -2.553569 & 2.347616 & -1.074523 \\
$\mathrm{H}$ & -2.430080 & 2.734738 & 0.658673 \\
$\mathrm{H}$ & -1.128188 & 3.229996 & -0.462155 \\
$\mathrm{C}$ & 1.864820 & -0.207913 & 1.785199 \\
$\mathrm{H}$ & 2.439988 & 0.713087 & 1.924089 \\
$\mathrm{H}$ & 2.546025 & -1.021211 & 1.535256 \\
$\mathrm{H}$ & 1.362755 & -0.474372 & 2.721172 \\
$\mathrm{H}$ & 0.447574 & 2.083701 & 0.842438 \\
$\mathrm{Br}$ & 1.813473 & 0.329429 & -1.031450 \\
$\mathrm{O}$ & -0.716817 & 1.043359 & 2.220855 \\
$\mathrm{H}$ & -0.068918 & 1.331706 & 2.883206
\end{tabular}


CIS-3 $\left(\mathrm{C}_{9} \mathrm{H}_{16} \mathrm{~N}_{2} \mathrm{O}_{2}\right)$

$\begin{array}{rrrr}\mathrm{N} & 1.185399 & 0.889348 & -0.343154 \\ \mathrm{C} & 0.035365 & 1.527436 & 0.211484 \\ \mathrm{~N} & -1.001697 & 0.699288 & 0.544285 \\ \mathrm{C} & -1.002783 & -0.736167 & 0.215609 \\ \mathrm{C} & 0.401942 & -1.292732 & 0.534425 \\ \mathrm{C} & 1.433487 & -0.457814 & -0.238816 \\ \mathrm{O} & 2.431143 & -0.996789 & -0.705318 \\ \mathrm{O} & 0.028129 & 2.742198 & 0.353081 \\ \mathrm{C} & 2.212007 & 1.755060 & -0.934334 \\ \mathrm{C} & -2.139601 & 1.265403 & 1.261939 \\ \mathrm{O} & 0.501389 & -2.628980 & 0.085857 \\ \mathrm{C} & 0.727146 & -1.234728 & 2.040613 \\ \mathrm{H} & -1.972135 & 2.335269 & 1.380459 \\ \mathrm{H} & -3.070529 & 1.106489 & 0.708328 \\ \mathrm{H} & -2.237011 & 0.798926 & 2.250692 \\ \mathrm{H} & 2.742370 & 1.182272 & -1.694474 \\ \mathrm{H} & 2.924128 & 2.089237 & -0.173049 \\ \mathrm{H} & 1.726208 & 2.627685 & -1.367445 \\ \mathrm{H} & 0.624551 & -0.228749 & 2.457843 \\ \mathrm{H} & 1.393310 & -2.693767 & -0.311299 \\ \mathrm{H} & 1.752254 & -1.581292 & 2.204342 \\ \mathrm{H} & 0.049428 & -1.911091 & 2.571453 \\ \mathrm{H} & -1.715704 & -1.210086 & 0.901166 \\ \mathrm{C} & -1.440412 & -1.034047 & -1.231807 \\ \mathrm{C} & -2.857677 & -0.568269 & -1.580559 \\ \mathrm{H} & -0.725726 & -0.572602 & -1.926044 \\ \mathrm{H} & -1.354227 & -2.115156 & -1.373232 \\ \mathrm{H} & -3.118330 & -0.875458 & -2.599287 \\ \mathrm{H} & -3.604036 & -1.005895 & -0.905627 \\ \mathrm{H} & -2.953674 & 0.521952 & -1.534166\end{array}$

\section{TRANS-3 $\left(\mathrm{C}_{9} \mathrm{H}_{16} \mathrm{~N}_{2} \mathrm{O}_{2}\right)$}

$\begin{array}{lrrr}\mathrm{N} & 0.971001 & -1.135690 & -0.349496 \\ \mathrm{C} & -0.290463 & -1.499981 & 0.178074 \\ \mathrm{~N} & -1.079804 & -0.461873 & 0.621191 \\ \mathrm{C} & -0.799098 & 0.927394 & 0.213040 \\ \mathrm{C} & 0.692064 & 1.177969 & 0.523172 \\ \mathrm{C} & 1.544503 & 0.131260 & -0.230260 \\ \mathrm{O} & 2.659955 & 0.369370 & -0.656806 \\ \mathrm{O} & -0.625547 & -2.673625 & 0.251913 \\ \mathrm{C} & 1.783251 & -2.214262 & -0.925317 \\ \mathrm{C} & -2.364854 & -0.821078 & 1.218708 \\ \mathrm{O} & 0.920062 & 0.998516 & 1.925684 \\ \mathrm{C} & 1.169995 & 2.585811 & 0.197571 \\ \mathrm{H} & -2.231712 & -1.706388 & 1.842385 \\ \mathrm{H} & -2.709201 & 0.013866 & 1.835093 \\ \mathrm{H} & -3.129500 & -1.047758 & 0.467555 \\ \mathrm{H} & 2.471657 & -1.769998 & -1.642429 \\ \mathrm{H} & 1.121616 & -2.933969 & -1.404805 \\ \mathrm{H} & 2.357257 & -2.726099 & -0.146166 \\ \mathrm{H} & 1.166456 & 2.779573 & -0.877002 \\ \mathrm{H} & 0.413704 & 0.212716 & 2.195796 \\ \mathrm{H} & 2.194816 & 2.700537 & 0.555409 \\ \mathrm{H} & 0.532026 & 3.317558 & 0.703197 \\ \mathrm{H} & -1.375225 & 1.566998 & 0.891916 \\ \mathrm{C} & -1.204224 & 1.256280 & -1.239412 \\ & & \mathrm{~S} 44 & \\ & & & \end{array}$




$\begin{array}{rrrr}\text { C } & -2.679983 & 1.005851 & -1.572715 \\ \text { H } & -2.927325 & -0.060347 & -1.574629 \\ \text { H } & -3.346099 & 1.507459 & -0.860108 \\ \text { H } & -0.980473 & 2.315537 & -1.411849 \\ \text { H } & -0.574452 & 0.690406 & -1.937836 \\ \text { H } & -2.910354 & 1.395531 & -2.569987\end{array}$

CIS-4 $\left(\mathrm{C}_{11} \mathrm{H}_{20} \mathrm{~N}_{2} \mathrm{O}_{3}\right)$

$\begin{array}{rrrr}\mathrm{N} & 1.651531 & 0.720758 & -0.853279 \\ \mathrm{C} & 0.980089 & 1.566364 & 0.080022 \\ \mathrm{~N} & 0.146408 & 0.934696 & 0.961037 \\ \mathrm{C} & -0.170302 & -0.500296 & 0.855385 \\ \mathrm{C} & 1.147461 & -1.248766 & 0.564464 \\ \mathrm{C} & 1.775924 & -0.638284 & -0.696804 \\ \mathrm{O} & 2.372771 & -1.355854 & -1.491983 \\ \mathrm{O} & 1.173309 & 2.773634 & 0.047959 \\ \mathrm{C} & 2.359276 & 1.375779 & -1.959865 \\ \mathrm{C} & -0.434833 & 1.716162 & 2.047558 \\ \mathrm{O} & 0.880300 & -2.610669 & 0.300800 \\ \mathrm{C} & 2.148426 & -1.142797 & 1.732768 \\ \mathrm{H} & -0.109038 & 2.749490 & 1.935827 \\ \mathrm{H} & -1.528316 & 1.678802 & 2.016983 \\ \mathrm{H} & -0.098018 & 1.329252 & 3.017860 \\ \mathrm{H} & 2.403353 & 0.676593 & -2.794341 \\ \mathrm{H} & 3.376586 & 1.648300 & -1.661079 \\ \mathrm{H} & 1.818902 & 2.280804 & -2.232100 \\ \mathrm{H} & 2.362719 & -0.108229 & 2.017274 \\ \mathrm{H} & 1.468552 & -2.841519 & -0.446423 \\ \mathrm{H} & 3.087119 & -1.631247 & 1.453204 \\ \mathrm{H} & 1.734131 & -1.670797 & 2.597727 \\ \mathrm{H} & -0.525305 & -0.812472 & 1.844756 \\ \mathrm{C} & -1.270063 & -0.811337 & -0.180080 \\ \mathrm{H} & -1.377668 & -1.900390 & -0.214267 \\ \mathrm{H} & -0.925272 & -0.503451 & -1.177165 \\ \mathrm{C} & -2.629838 & -0.162902 & 0.106417 \\ \mathrm{H} & -2.965183 & -0.436029 & 1.118783 \\ \mathrm{H} & -2.536577 & 0.931442 & 0.092521 \\ \mathrm{C} & -3.706772 & -0.579845 & -0.905135 \\ \mathrm{H} & -3.807992 & -1.674038 & -0.894343 \\ \mathrm{H} & -3.370782 & -0.315070 & -1.917593 \\ \mathrm{C} & -5.068635 & 0.065685 & -0.632431 \\ \mathrm{H} & -5.448776 & -0.213195 & 0.358083 \\ \mathrm{H} & -5.813275 & -0.245475 & -1.373551 \\ \mathrm{H} & -5.003050 & 1.160098 & -0.665796\end{array}$

TRANS-4 $\left(\mathrm{C}_{11} \mathrm{H}_{20} \mathrm{~N}_{2} \mathrm{O}_{3}\right)$

$\begin{array}{rrrr}\mathrm{N} & 1.588447 & 0.788273 & 0.842540 \\ \mathrm{C} & 0.865300 & 1.563528 & -0.095071 \\ \mathrm{~N} & 0.159989 & 0.852600 & -1.041877 \\ \mathrm{C} & -0.132200 & -0.580640 & -0.843001 \\ \mathrm{C} & 1.219830 & -1.254908 & -0.527469 \\ \mathrm{C} & 1.833304 & -0.579826 & 0.719033 \\ \mathrm{O} & 2.522022 & -1.177816 & 1.525947 \\ \mathrm{O} & 0.902381 & 2.785308 & -0.062779 \\ \mathrm{C} & 2.263964 & 1.514089 & 1.924781 \\ \mathrm{C} & -0.550178 & 1.623095 & -2.061015 \\ \mathrm{O} & 2.118710 & -1.048852 & -1.623797 \\ & & \mathrm{~S} 45 & \end{array}$




$\begin{array}{rrrr}\mathrm{C} & 1.140939 & -2.762759 & -0.332265 \\ \mathrm{H} & 0.035149 & 2.511435 & -2.300709 \\ \mathrm{H} & -0.662998 & 1.003544 & -2.955478 \\ \mathrm{H} & -1.542700 & 1.948089 & -1.729080 \\ \mathrm{H} & 2.340256 & 0.849319 & 2.784227 \\ \mathrm{H} & 1.683148 & 2.403430 & 2.162938 \\ \mathrm{H} & 3.270479 & 1.817471 & 1.618684 \\ \mathrm{H} & 0.583795 & -3.031253 & 0.567927 \\ \mathrm{H} & 1.995834 & -0.130441 & -1.920885 \\ \mathrm{H} & 2.153991 & -3.156173 & -0.229321 \\ \mathrm{H} & 0.668646 & -3.222709 & -1.206296 \\ \mathrm{H} & -0.459160 & -0.962999 & -1.817149 \\ \mathrm{C} & -1.233777 & -0.863140 & 0.200806 \\ \mathrm{C} & -2.582095 & -0.180740 & -0.069417 \\ \mathrm{C} & -3.678242 & -0.632490 & 0.906075 \\ \mathrm{C} & -5.019136 & 0.067984 & 0.663877 \\ \mathrm{H} & -4.924377 & 1.155209 & 0.772686 \\ \mathrm{H} & -5.781088 & -0.272073 & 1.373966 \\ \mathrm{H} & -5.393686 & -0.132757 & -0.347304 \\ \mathrm{H} & -3.810727 & -1.720377 & 0.822229 \\ \mathrm{H} & -3.345473 & -0.444831 & 1.936468 \\ \mathrm{H} & -2.470662 & 0.908636 & 0.003451 \\ \mathrm{H} & -2.907436 & -0.394113 & -1.098586 \\ \mathrm{H} & -1.389328 & -1.948644 & 0.230438 \\ \mathrm{H} & -0.879093 & -0.575439 & 1.199656\end{array}$

\section{CIS-5 $\left(\mathrm{C}_{10} \mathrm{H}_{18} \mathrm{~N}_{2} \mathrm{O}_{3}\right)$}

$\begin{array}{lrrr}\mathrm{N} & 1.478444 & 0.619309 & -0.393005 \\ \mathrm{C} & 0.508431 & 1.553139 & 0.069500 \\ \mathrm{~N} & -0.646592 & 1.038505 & 0.589041 \\ \mathrm{C} & -1.001462 & -0.391432 & 0.546209 \\ \mathrm{C} & 0.292196 & -1.203118 & 0.788894 \\ \mathrm{C} & 1.410272 & -0.727512 & -0.148193 \\ \mathrm{O} & 2.213589 & -1.540374 & -0.595620 \\ \mathrm{O} & 0.735340 & 2.752790 & -0.006949 \\ \mathrm{C} & 2.619831 & 1.161744 & -1.138846 \\ \mathrm{C} & -1.583609 & 2.019833 & 1.136350 \\ \mathrm{O} & 0.057635 & -2.570711 & 0.525049 \\ \mathrm{C} & 0.786339 & -1.041082 & 2.243852 \\ \mathrm{H} & -2.317396 & 1.496011 & 1.752323 \\ \mathrm{H} & -1.043319 & 2.744484 & 1.749898 \\ \mathrm{H} & -2.107526 & 2.571990 & 0.348624 \\ \mathrm{H} & 3.040683 & 0.355706 & -1.738493 \\ \mathrm{H} & 3.381469 & 1.544952 & -0.452629 \\ \mathrm{H} & 2.276401 & 1.981110 & -1.769423 \\ \mathrm{H} & 0.920663 & 0.006841 & 2.529248 \\ \mathrm{H} & 0.881311 & -2.885811 & 0.100096 \\ \mathrm{H} & 1.739154 & -1.564686 & 2.370477 \\ \mathrm{H} & 0.052218 & -1.500685 & 2.913302 \\ \mathrm{C} & -1.827370 & -0.839005 & -0.695042 \\ \mathrm{C} & -3.227878 & -0.204912 & -0.705135 \\ \mathrm{C} & -1.138546 & -0.628411 & -2.051866 \\ \mathrm{H} & -1.642283 & -0.566272 & 1.419256 \\ \mathrm{H} & -0.925447 & 0.430284 & -2.241912 \\ \mathrm{H} & -1.798118 & -0.974598 & -2.856109 \\ \mathrm{H} & -0.204153 & -1.191523 & -2.139305 \\ \mathrm{H} & -3.846713 & -0.678023 & -1.475900 \\ \mathrm{H} & -3.744755 & -0.334201 & 0.253977\end{array}$




$\begin{array}{rrrr}\mathrm{H} & -3.195058 & 0.867098 & -0.931115 \\ \mathrm{H} & -1.953626 & -1.917785 & -0.551674\end{array}$

TRANS-5 $\left(\mathrm{C}_{10} \mathrm{H}_{18} \mathrm{~N}_{2} \mathrm{O}_{3}\right)$

$\begin{array}{lrrr}\mathrm{N} & 1.451111 & 0.535406 & 0.471518 \\ \mathrm{C} & 0.590269 & 1.531039 & -0.065179 \\ \mathrm{~N} & -0.626795 & 1.099144 & -0.521751 \\ \mathrm{C} & -1.033533 & -0.314309 & -0.496816 \\ \mathrm{C} & 0.197715 & -1.198075 & -0.762772 \\ \mathrm{C} & 1.371614 & -0.804813 & 0.150916 \\ \mathrm{O} & 2.220317 & -1.613672 & 0.500748 \\ \mathrm{O} & 0.951495 & 2.700081 & -0.072550 \\ \mathrm{C} & 2.643930 & 1.010722 & 1.180466 \\ \mathrm{C} & -1.383921 & 2.015388 & -1.372365 \\ \mathrm{O} & 0.598848 & -0.853926 & -2.103285 \\ \mathrm{C} & -0.081684 & -2.696432 & -0.673063 \\ \mathrm{H} & -0.939920 & 3.005802 & -1.285758 \\ \mathrm{H} & -1.340074 & 1.685120 & -2.418279 \\ \mathrm{H} & -2.429624 & 2.060278 & -1.057354 \\ \mathrm{H} & 3.443875 & 1.256530 & 0.474824 \\ \mathrm{H} & 2.983068 & 0.216247 & 1.844043 \\ \mathrm{H} & 2.384894 & 1.907973 & 1.740276 \\ \mathrm{H} & -0.334435 & -3.013775 & 0.341738 \\ \mathrm{H} & 1.394784 & -1.373000 & -2.306769 \\ \mathrm{H} & -1.673921 & -0.454543 & -1.375715 \\ \mathrm{H} & 0.814295 & -3.250802 & -0.966218 \\ \mathrm{H} & -0.901970 & -2.959379 & -1.348411 \\ \mathrm{C} & -1.889607 & -0.698836 & 0.745979 \\ \mathrm{C} & -1.162510 & -0.626333 & 2.098631 \\ \mathrm{C} & -3.181277 & 0.132979 & 0.803957 \\ \mathrm{H} & -0.822383 & 0.392213 & 2.315771 \\ \mathrm{H} & -2.965445 & 1.186276 & 1.014787 \\ \mathrm{H} & -3.745590 & 0.078623 & -0.134843 \\ \mathrm{H} & -3.833234 & -0.236198 & 1.603607 \\ \mathrm{H} & -1.850785 & -0.919098 & 2.899937 \\ \mathrm{H} & -0.296043 & -1.293256 & 2.154298 \\ \mathrm{H} & -2.190478 & -1.742177 & 0.581187\end{array}$

\section{CIS-6 $\left(\mathrm{C}_{13} \mathrm{H}_{22} \mathrm{~N}_{2} \mathrm{O}_{3}\right)$}

$\begin{array}{lrrr}\mathrm{N} & 1.930764 & 0.062137 & -0.971144 \\ \mathrm{C} & 1.474378 & 1.340125 & -0.542521 \\ \mathrm{~N} & 0.609298 & 1.369357 & 0.514603 \\ \mathrm{C} & 0.006740 & 0.166504 & 1.116691 \\ \mathrm{C} & 1.083743 & -0.945568 & 1.124509 \\ \mathrm{C} & 1.716424 & -1.093732 & -0.266428 \\ \mathrm{O} & 2.038870 & -2.207980 & -0.668097 \\ \mathrm{O} & 1.865591 & 2.345278 & -1.119927 \\ \mathrm{C} & 2.672418 & 0.026129 & -2.236474 \\ \mathrm{C} & 0.231566 & 2.704679 & 0.978964 \\ \mathrm{O} & 0.493965 & -2.183326 & 1.463546 \\ \mathrm{C} & 2.205351 & -0.624962 & 2.136484 \\ \mathrm{H} & -0.436843 & 3.208777 & 0.272756 \\ \mathrm{H} & -0.274580 & 2.609225 & 1.941438 \\ \mathrm{H} & 1.124217 & 3.324710 & 1.094715 \\ \mathrm{H} & 2.903223 & -1.016078 & -2.449384 \\ \mathrm{H} & 3.588889 & 0.613816 & -2.149570 \\ \mathrm{H} & 2.062455 & 0.451271 & -3.036649\end{array}$




$\begin{array}{rrrr}\mathrm{H} & 2.645854 & 0.364211 & 1.978574 \\ \mathrm{H} & 0.945647 & -2.838324 & 0.893254 \\ \mathrm{H} & 2.995315 & -1.379322 & 2.064408 \\ \mathrm{H} & 1.782050 & -0.668815 & 3.145197 \\ \mathrm{H} & -0.177540 & 0.418996 & 2.168753 \\ \mathrm{C} & -1.366778 & -0.263835 & 0.528894 \\ \mathrm{C} & -3.862697 & 0.227020 & 0.442766 \\ \mathrm{C} & -2.769931 & -1.081477 & -1.427387 \\ \mathrm{C} & -3.891257 & -0.101724 & -1.056379 \\ \mathrm{C} & -1.384712 & -0.579927 & -0.980476 \\ \mathrm{C} & -2.481272 & 0.747821 & 0.868485 \\ \mathrm{H} & -4.099431 & -0.679368 & 1.018859 \\ \mathrm{H} & -2.966091 & -2.051033 & -0.947776 \\ \mathrm{H} & -3.771878 & 0.827121 & -1.633811 \\ \mathrm{H} & -1.125387 & 0.322461 & -1.553242 \\ \mathrm{H} & -2.294571 & 1.701448 & 0.355640 \\ \mathrm{H} & -1.600704 & -1.196190 & 1.058011 \\ \mathrm{H} & -4.635257 & 0.966884 & 0.688416 \\ \mathrm{H} & -2.765327 & -1.264238 & -2.509421 \\ \mathrm{H} & -4.866939 & -0.518769 & -1.337214 \\ \mathrm{H} & -0.640745 & -1.345715 & -1.227702 \\ \mathrm{H} & -2.478805 & 0.962481 & 1.946240\end{array}$

\section{TRANS-6 $\left(\mathrm{C}_{13} \mathrm{H}_{22} \mathrm{~N}_{2} \mathrm{O}_{3}\right)$}

$\begin{array}{lrrr}\mathrm{N} & 1.805093 & 0.022325 & 1.043934 \\ \mathrm{C} & 1.442379 & 1.330274 & 0.619248 \\ \mathrm{~N} & 0.542483 & 1.405128 & -0.410589 \\ \mathrm{C} & 0.000729 & 0.215653 & -1.087798 \\ \mathrm{C} & 1.089883 & -0.869569 & -1.145351 \\ \mathrm{C} & 1.723911 & -1.095565 & 0.238010 \\ \mathrm{O} & 2.197378 & -2.174647 & 0.567058 \\ \mathrm{O} & 1.913012 & 2.304003 & 1.191540 \\ \mathrm{C} & 2.560506 & -0.082073 & 2.296318 \\ \mathrm{C} & 0.426567 & 2.691418 & -1.096294 \\ \mathrm{O} & 2.122120 & -0.290981 & -1.969241 \\ \mathrm{C} & 0.622208 & -2.189441 & -1.753076 \\ \mathrm{H} & 0.786703 & 3.472199 & -0.427916 \\ \mathrm{H} & 1.029431 & 2.691038 & -2.014151 \\ \mathrm{H} & -0.615988 & 2.888266 & -1.357001 \\ \mathrm{H} & 2.392260 & -1.074286 & 2.713735 \\ \mathrm{H} & 2.216559 & 0.696101 & 2.975336 \\ \mathrm{H} & 3.632421 & 0.053642 & 2.118708 \\ \mathrm{H} & -0.128094 & -2.691088 & -1.136466 \\ \mathrm{H} & 2.850847 & -0.932521 & -2.007556 \\ \mathrm{H} & 1.470764 & -2.874106 & -1.840366 \\ \mathrm{H} & 0.207106 & -2.009775 & -2.749984 \\ \mathrm{H} & -0.149882 & 0.512820 & -2.132645 \\ \mathrm{C} & -1.387689 & -0.238151 & -0.550132 \\ \mathrm{C} & -2.813134 & -1.246020 & 1.305973 \\ \mathrm{C} & -3.847874 & 0.389163 & -0.324256 \\ \mathrm{C} & -3.870893 & -0.151384 & 1.111881 \\ \mathrm{C} & -2.444251 & 0.873535 & -0.719655 \\ \mathrm{C} & -1.409488 & -0.763288 & 0.900734 \\ \mathrm{H} & -3.082510 & -2.122637 & 0.698000 \\ \mathrm{H} & -4.164071 & -0.405645 & -1.016343 \\ \mathrm{H} & -3.673390 & 0.672716 & 1.813079 \\ \mathrm{H} & -2.168478 & 1.730785 & -0.090741 \\ & & \mathrm{~S} 48 & \\ & & & \end{array}$




$\begin{array}{rrrr} & & & \\ \mathrm{H} & -1.094524 & 0.038809 & 1.581895 \\ \mathrm{H} & -1.698716 & -1.065727 & -1.204631 \\ \mathrm{H} & -2.799726 & -1.585049 & 2.349430 \\ \mathrm{H} & -4.571574 & 1.206159 & -0.437441 \\ \mathrm{H} & -4.868038 & -0.537939 & 1.357481 \\ \mathrm{H} & -2.452669 & 1.229613 & -1.759207 \\ \mathrm{H} & -0.695626 & -1.585334 & 1.030879\end{array}$

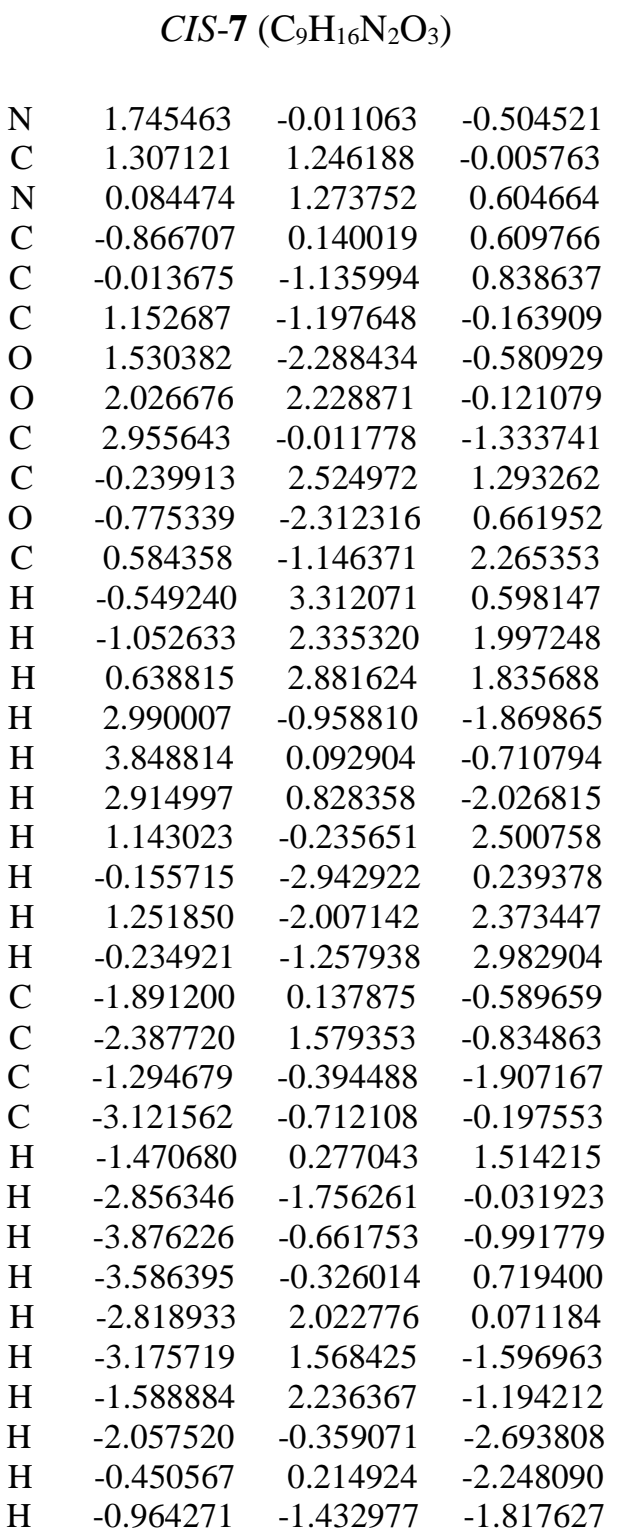

\section{TRANS-7 $\left(\mathrm{C}_{9} \mathrm{H}_{16} \mathrm{~N}_{2} \mathrm{O}_{3}\right)$}

$\begin{array}{rrrr}\mathrm{N} & 1.682368 & 0.006451 & 0.590407 \\ \mathrm{C} & 1.303698 & 1.249969 & 0.018243 \\ \mathrm{~N} & 0.065266 & 1.294093 & -0.562250 \\ \mathrm{C} & -0.856294 & 0.141607 & -0.586253 \\ \mathrm{C} & 0.005758 & -1.121455 & -0.834100 \\ \mathrm{C} & 1.164525 & -1.205014 & 0.179992 \\ \mathrm{O} & 1.635938 & -2.274936 & 0.539274\end{array}$




$\begin{array}{rrrr}\mathrm{O} & 2.069021 & 2.202583 & 0.084722 \\ \mathrm{C} & 2.885992 & 0.006488 & 1.427883 \\ \mathrm{C} & -0.197813 & 2.399064 & -1.481786 \\ \mathrm{O} & 0.611352 & -0.859944 & -2.118643 \\ \mathrm{C} & -0.730052 & -2.458289 & -0.898022 \\ \mathrm{H} & 0.548570 & 3.173112 & -1.309445 \\ \mathrm{H} & -0.131537 & 2.050656 & -2.520371 \\ \mathrm{H} & -1.193071 & 2.815123 & -1.307779 \\ \mathrm{H} & 3.791019 & -0.004139 & 0.812244 \\ \mathrm{H} & 2.863172 & -0.886765 & 2.050501 \\ \mathrm{H} & 2.889917 & 0.910000 & 2.036271 \\ \mathrm{H} & -1.120108 & -2.769901 & 0.072630 \\ \mathrm{H} & 1.179194 & -1.620467 & -2.326149 \\ \mathrm{H} & -1.445453 & 0.260520 & -1.502559 \\ \mathrm{H} & -0.025739 & -3.233861 & -1.213455 \\ \mathrm{H} & -1.548476 & -2.404213 & -1.621254 \\ \mathrm{C} & -1.891539 & 0.158181 & 0.609001 \\ \mathrm{C} & -3.130464 & -0.703851 & 0.266440 \\ \mathrm{C} & -1.293554 & -0.310617 & 1.951633 \\ \mathrm{C} & -2.409600 & 1.602135 & 0.797343 \\ \mathrm{H} & -2.932574 & -1.776897 & 0.278783 \\ \mathrm{H} & -0.914720 & -1.337629 & 1.912015 \\ \mathrm{H} & -0.475559 & 0.337977 & 2.280084 \\ \mathrm{H} & -1.604147 & 2.304109 & 1.029025 \\ \mathrm{H} & -3.125048 & 1.625762 & 1.627066 \\ \mathrm{H} & -2.068278 & -0.280229 & 2.726966 \\ \mathrm{H} & -3.920873 & -0.514069 & 1.001749 \\ \mathrm{H} & -3.533794 & -0.443739 & -0.720379 \\ \mathrm{H} & -2.935998 & 1.959940 & -0.095882 \\ & & & \end{array}$

\section{CIS-8 $\left(\mathrm{C}_{9} \mathrm{H}_{14} \mathrm{~N}_{2} \mathrm{O}_{3}\right)$}

$\begin{array}{lrrr}\mathrm{N} & -0.030224 & -1.419253 & 0.172220 \\ \mathrm{C} & -1.240504 & -0.829126 & -0.290782 \\ \mathrm{~N} & -1.199241 & 0.517858 & -0.540795 \\ \mathrm{C} & -0.070203 & 1.357917 & -0.142249 \\ \mathrm{C} & 1.239549 & 0.606384 & -0.476601 \\ \mathrm{C} & 1.186066 & -0.781160 & 0.175115 \\ \mathrm{O} & 2.211407 & -1.278005 & 0.627307 \\ \mathrm{O} & -2.232935 & -1.527258 & -0.439883 \\ \mathrm{C} & -0.108971 & -2.809046 & 0.639180 \\ \mathrm{C} & -2.413465 & 1.182009 & -1.003197 \\ \mathrm{O} & 2.337205 & 1.305312 & 0.070252 \\ \mathrm{C} & 1.436552 & 0.445170 & -1.996534 \\ \mathrm{H} & -3.167574 & 0.422134 & -1.202462 \\ \mathrm{H} & -2.790408 & 1.876417 & -0.243103 \\ \mathrm{H} & -2.205978 & 1.743922 & -1.921472 \\ \mathrm{H} & 0.747879 & -2.990343 & 1.286226 \\ \mathrm{H} & -0.083524 & -3.500467 & -0.208486 \\ \mathrm{H} & -1.044138 & -2.952377 & 1.179067 \\ \mathrm{H} & 0.578807 & -0.027384 & -2.484729 \\ \mathrm{H} & 2.918680 & 0.607784 & 0.435278 \\ \mathrm{H} & 2.330113 & -0.157350 & -2.188225 \\ \mathrm{H} & 1.594075 & 1.436334 & -2.434226 \\ \mathrm{C} & -0.122789 & 1.821931 & 1.301182 \\ \mathrm{C} & -1.023058 & 1.442174 & 2.206676 \\ \mathrm{H} & -0.095793 & 2.248344 & -0.784618 \\ \mathrm{H} & 0.665380 & 2.520464 & 1.569174 \\ & & \mathrm{~S} 50 & \\ & & & \end{array}$




$\begin{array}{llll}\mathrm{H} & -0.991628 & 1.825207 & 3.222578 \\ \mathrm{H} & -1.820485 & 0.740375 & 1.977222\end{array}$

\section{TRANS-8 $\left(\mathrm{C}_{9} \mathrm{H}_{14} \mathrm{~N}_{2} \mathrm{O}_{3}\right)$}

$\begin{array}{lrrr}\mathrm{N} & 1.504884 & -0.161344 & 0.327928 \\ \mathrm{C} & 1.121942 & 1.140055 & -0.069813 \\ \mathrm{~N} & -0.170999 & 1.280161 & -0.544417 \\ \mathrm{C} & -1.194225 & 0.270414 & -0.205602 \\ \mathrm{C} & -0.605988 & -1.107933 & -0.574071 \\ \mathrm{C} & 0.727192 & -1.312849 & 0.175900 \\ \mathrm{O} & 1.106223 & -2.403483 & 0.560946 \\ \mathrm{O} & 1.923913 & 2.059780 & -0.013647 \\ \mathrm{C} & 2.863484 & -0.285126 & 0.871873 \\ \mathrm{C} & -0.638295 & 2.639054 & -0.812493 \\ \mathrm{O} & -0.321068 & -1.131009 & -1.975354 \\ \mathrm{C} & -1.561526 & -2.257286 & -0.299308 \\ \mathrm{H} & 0.219090 & 3.255507 & -1.078615 \\ \mathrm{H} & -1.349756 & 2.615639 & -1.644210 \\ \mathrm{H} & -1.132293 & 3.076273 & 0.064788 \\ \mathrm{H} & 2.974332 & 0.354400 & 1.750162 \\ \mathrm{H} & 3.596478 & 0.024587 & 0.123119 \\ \mathrm{H} & 3.009599 & -1.329052 & 1.140962 \\ \mathrm{H} & -1.820955 & -2.312685 & 0.760478 \\ \mathrm{H} & 0.081725 & -0.272150 & -2.195024 \\ \mathrm{H} & -1.087379 & -3.196353 & -0.589685 \\ \mathrm{H} & -2.474781 & -2.117999 & -0.886089 \\ \mathrm{H} & -2.039532 & 0.440644 & -0.882000 \\ \mathrm{C} & -1.666517 & 0.409632 & 1.222706 \\ \mathrm{C} & -2.911325 & 0.741417 & 1.566288 \\ \mathrm{H} & -0.916698 & 0.252319 & 1.998308 \\ \mathrm{H} & -3.207456 & 0.849138 & 2.606031 \\ \mathrm{H} & -3.684688 & 0.913845 & 0.820186\end{array}$

\section{CIS-9 $\left(\mathrm{C}_{13} \mathrm{H}_{16} \mathrm{~N}_{2} \mathrm{O}_{3}\right)$}

$\begin{array}{lrrr}\mathrm{N} & 1.822271 & 0.219204 & 0.877621 \\ \mathrm{C} & 1.656744 & 1.220865 & -0.120814 \\ \mathrm{~N} & 0.786626 & 0.930978 & -1.140795 \\ \mathrm{C} & -0.127361 & -0.212682 & -1.096297 \\ \mathrm{C} & 0.666127 & -1.430184 & -0.547927 \\ \mathrm{C} & 1.282505 & -1.042720 & 0.799376 \\ \mathrm{O} & 1.315058 & -1.855874 & 1.715379 \\ \mathrm{O} & 2.276740 & 2.270241 & -0.023994 \\ \mathrm{C} & 2.590125 & 0.610025 & 2.065980 \\ \mathrm{C} & 0.524138 & 1.965876 & -2.135660 \\ \mathrm{C} & -1.436291 & 0.076751 & -0.359386 \\ \mathrm{C} & -2.548962 & -0.742506 & -0.600425 \\ \mathrm{C} & -3.758795 & -0.510961 & 0.050662 \\ \mathrm{C} & -3.881524 & 0.551653 & 0.949429 \\ \mathrm{C} & -2.784738 & 1.377500 & 1.188620 \\ \mathrm{C} & -1.570665 & 1.142114 & 0.538166 \\ \mathrm{C} & 1.792949 & -1.846915 & -1.515747 \\ \mathrm{O} & -0.193132 & -2.527180 & -0.347921 \\ \mathrm{H} & 2.367924 & -2.662423 & -1.067030 \\ \mathrm{H} & 2.468936 & -1.022667 & -1.757287\end{array}$




$\begin{array}{rrrr}\mathrm{H} & 1.339367 & -2.218536 & -2.440247 \\ \mathrm{H} & -0.376482 & -0.453776 & -2.136691 \\ \mathrm{H} & 1.300036 & 2.726290 & -2.060058 \\ \mathrm{H} & -0.454733 & 2.433147 & -1.969654 \\ \mathrm{H} & 0.537650 & 1.522120 & -3.137115 \\ \mathrm{H} & -2.869093 & 2.212106 & 1.879703 \\ \mathrm{H} & -4.826503 & 0.736406 & 1.453486 \\ \mathrm{H} & -4.608749 & -1.158714 & -0.147643 \\ \mathrm{H} & -2.456598 & -1.574595 & -1.291370 \\ \mathrm{H} & 2.714343 & -0.277637 & 2.683827 \\ \mathrm{H} & 2.055524 & 1.381310 & 2.627081 \\ \mathrm{H} & 3.556749 & 1.010077 & 1.757581 \\ \mathrm{H} & -0.735605 & 1.809938 & 0.728813 \\ \mathrm{H} & 0.017466 & -2.858760 & 0.548302\end{array}$

TRANS-9 $\left(\mathrm{C}_{13} \mathrm{H}_{16} \mathrm{~N}_{2} \mathrm{O}_{3}\right)$

$\begin{array}{lrrr}\mathrm{N} & 1.918026 & 0.294754 & 0.758715 \\ \mathrm{C} & 1.587545 & 1.289953 & -0.188450 \\ \mathrm{~N} & 0.688380 & 0.935054 & -1.175199 \\ \mathrm{C} & -0.179545 & -0.245479 & -1.046866 \\ \mathrm{C} & 0.703288 & -1.419058 & -0.555364 \\ \mathrm{C} & 1.464416 & -1.024759 & 0.726205 \\ \mathrm{O} & 1.720623 & -1.818775 & 1.612242 \\ \mathrm{O} & 2.108255 & 2.394116 & -0.122764 \\ \mathrm{C} & 2.814195 & 0.715902 & 1.842872 \\ \mathrm{C} & 0.233251 & 2.004634 & -2.062872 \\ \mathrm{C} & -1.465574 & 0.011116 & -0.264986 \\ \mathrm{C} & -2.674296 & -0.496094 & -0.761419 \\ \mathrm{C} & -3.874167 & -0.292875 & -0.079573 \\ \mathrm{C} & -3.885110 & 0.432095 & 1.112469 \\ \mathrm{C} & -2.690330 & 0.952217 & 1.611362 \\ \mathrm{C} & -1.491262 & 0.745396 & 0.928488 \\ \mathrm{O} & 1.687471 & -1.696580 & -1.559714 \\ \mathrm{C} & -0.069432 & -2.710713 & -0.342448 \\ \mathrm{H} & 1.050888 & 2.708048 & -2.212838 \\ \mathrm{H} & -0.061095 & 1.566649 & -3.021219 \\ \mathrm{H} & -0.623861 & 2.542859 & -1.639017 \\ \mathrm{H} & 3.777047 & 1.028226 & 1.432537 \\ \mathrm{H} & 2.939339 & -0.136822 & 2.507136 \\ \mathrm{H} & 2.378870 & 1.559901 & 2.382613 \\ \mathrm{H} & -0.802463 & -2.606201 & 0.459625 \\ \mathrm{H} & -0.578012 & 1.170843 & 1.331875 \\ \mathrm{H} & -2.688656 & 1.525829 & 2.534053 \\ \mathrm{H} & -4.817522 & 0.597236 & 1.645262 \\ \mathrm{H} & -4.800112 & -0.692078 & -0.484918 \\ \mathrm{H} & -2.677272 & -1.053403 & -1.695561 \\ \mathrm{H} & 2.037523 & -0.840960 & -1.863785 \\ \mathrm{H} & -0.463928 & -0.524713 & -2.068016 \\ \mathrm{H} & 0.630969 & -3.502700 & -0.071417 \\ \mathrm{H} & -0.582479 & -2.987871 & -1.268488\end{array}$

\section{$C I S-10\left(\mathrm{C}_{11} \mathrm{H}_{14} \mathrm{~N}_{2} \mathrm{O}_{3} \mathrm{~S}\right)$}
N 1.792783
0.077407
$-0.923065$
C 1.729178
$1.154528 \quad 0.004180$ 


$\begin{array}{lrrr}\mathrm{N} & 0.865336 & 0.998757 & 1.060454 \\ \mathrm{C} & -0.138074 & -0.066088 & 1.075414 \\ \mathrm{C} & 0.566033 & -1.377939 & 0.647868 \\ \mathrm{C} & 1.184760 & -1.142848 & -0.733528 \\ \mathrm{O} & 1.148015 & -2.027042 & -1.580159 \\ \mathrm{O} & 2.424166 & 2.144202 & -0.172133 \\ \mathrm{C} & 2.539496 & 0.331840 & -2.161397 \\ \mathrm{C} & 0.740311 & 2.088236 & 2.023740 \\ \mathrm{C} & 1.664248 & -1.790540 & 1.647011 \\ \mathrm{O} & -0.377472 & -2.419367 & 0.554115 \\ \mathrm{C} & -1.353251 & 0.304250 & 0.240331 \\ \mathrm{C} & -1.539818 & 1.446847 & -0.497707 \\ \mathrm{C} & -2.831657 & 1.533518 & -1.098752 \\ \mathrm{C} & -3.620635 & 0.458783 & -0.800533 \\ \mathrm{~S} & -2.789638 & -0.693589 & 0.193767 \\ \mathrm{H} & -0.448116 & -0.199676 & 2.119198 \\ \mathrm{H} & 2.107184 & 1.184530 & -2.689740 \\ \mathrm{H} & 3.581619 & 0.557874 & -1.927015 \\ \mathrm{H} & 2.468052 & -0.565223 & -2.773408 \\ \mathrm{H} & -0.173137 & 2.671487 & 1.853260 \\ \mathrm{H} & 0.710915 & 1.675587 & 3.037755 \\ \mathrm{H} & 1.604548 & 2.742260 & 1.917276 \\ \mathrm{H} & 2.419118 & -1.013969 & 1.793604 \\ \mathrm{H} & 1.195569 & -2.019228 & 2.609660 \\ \mathrm{H} & 2.151907 & -2.699336 & 1.282286 \\ \mathrm{H} & -0.227655 & -2.829892 & -0.321592 \\ \mathrm{H} & -0.782894 & 2.216494 & -0.602097 \\ \mathrm{H} & -3.150594 & 2.361622 & -1.722452 \\ \mathrm{H} & -4.630356 & 0.255887 & -1.133069 \\ & & & \end{array}$

\section{TRANS-10 $\left(\mathrm{C}_{11} \mathrm{H}_{14} \mathrm{~N}_{2} \mathrm{O}_{3} \mathrm{~S}\right)$}

$\begin{array}{lrrr}\mathrm{N} & 1.985210 & 0.350740 & 0.636506 \\ \mathrm{C} & 1.528646 & 1.395528 & -0.215931 \\ \mathrm{~N} & 0.453274 & 1.104180 & -1.008186 \\ \mathrm{C} & -0.320409 & -0.128786 & -0.875744 \\ \mathrm{C} & 0.635779 & -1.311585 & -0.615118 \\ \mathrm{C} & 1.644467 & -0.981725 & 0.498010 \\ \mathrm{O} & 2.180923 & -1.862113 & 1.154817 \\ \mathrm{O} & 2.091501 & 2.481238 & -0.200291 \\ \mathrm{C} & 3.070998 & 0.688915 & 1.565034 \\ \mathrm{C} & -0.106225 & 2.162491 & -1.838398 \\ \mathrm{C} & -1.451898 & 0.006231 & 0.127736 \\ \mathrm{C} & -1.413129 & 0.436565 & 1.430190 \\ \mathrm{C} & -2.689604 & 0.426431 & 2.069092 \\ \mathrm{C} & -3.691939 & -0.009594 & 1.247675 \\ \mathrm{~S} & -3.088215 & -0.410767 & -0.326336 \\ \mathrm{C} & -0.093550 & -2.619228 & -0.323671 \\ \mathrm{H} & -0.732210 & -2.879294 & -1.173702 \\ \mathrm{H} & 0.639267 & -3.415179 & -0.165635 \\ \mathrm{H} & -0.707873 & -2.552436 & 0.577141 \\ \mathrm{O} & 1.406044 & -1.426052 & -1.823339 \\ \mathrm{H} & 2.011467 & -2.176137 & -1.701179 \\ \mathrm{H} & 0.614578 & 2.976831 & -1.887473 \\ \mathrm{H} & -0.296514 & 1.776297 & -2.845853 \\ \mathrm{H} & -0.751463 & -0.330152 & -1.863218 \\ \mathrm{H} & -4.745918 & -0.112785 & 1.468371 \\ \mathrm{H} & 2.937734 & 1.716690 & 1.896765 \\ & & \mathrm{~S} 53 & \\ & & & \end{array}$




$\begin{array}{lrrr}\mathrm{H} & 3.029604 & -0.006855 & 2.401948 \\ \mathrm{H} & 4.045208 & 0.599527 & 1.073488 \\ \mathrm{H} & -1.047546 & 2.539708 & -1.417803 \\ \mathrm{H} & -2.849713 & 0.734265 & 3.096756 \\ \mathrm{H} & -0.501179 & 0.755380 & 1.922548\end{array}$

\begin{tabular}{|c|c|c|c|}
\hline \multicolumn{4}{|c|}{$C I S-11\left(\mathrm{C}_{9} \mathrm{H}_{12} \mathrm{~N}_{2} \mathrm{O}_{3}\right)$} \\
\hline $\mathrm{N}$ & -0.261752 & -1.401579 & \\
\hline $\mathrm{C}$ & -1.388201 & -0.612337 & -0.29222 \\
\hline $\mathrm{N}$ & -1.152953 & 0.723197 & -0.5272 \\
\hline $\mathrm{C}$ & 0.086871 & 1.358718 & -0.06 \\
\hline $\mathrm{C}$ & 1.278062 & 0.460343 & -0.4 \\
\hline $\mathrm{C}$ & 1.035452 & -0.942181 & 0.0 \\
\hline $\mathrm{O}$ & 1.982477 & -1.600632 & \\
\hline 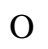 & -2.484151 & -1.139532 & -0.3 \\
\hline $\mathrm{C}$ & -0.547419 & -2.788279 & \\
\hline $\mathrm{C}$ & -2.309517 & 1.5 & \\
\hline $\mathrm{O}$ & 2.460624 & 0.976 & 0.0 \\
\hline $\mathrm{C}$ & 1.421793 & 0.3 & \\
\hline $\mathrm{H}$ & -3.134853 & 0.9 & \\
\hline$H$ & -2.611837 & 2.075192 & \\
\hline H & -2.055962 & 2.3 & -1.4 \\
\hline$\Pi$ & 0.399692 & -3.25 & 0.7 \\
\hline $\mathrm{H}$ & -1.016806 & -3.318369 & -0. \\
\hline 1 & -1.229183 & -2.802276 & \\
\hline $\mathrm{H}$ & 0.500508 & 0.055513 & -2.4 \\
\hline $\mathrm{H}$ & 2.933832 & 0.201057 & 0.4 \\
\hline- & 2.223526 & -0.326323 & -2.2 \\
\hline $\mathrm{H}$ & 1.705312 & 1.363819 & -2.3 \\
\hline $\mathrm{C}$ & 0.051113 & 1.643144 & 1.38038 \\
\hline 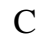 & -0.039831 & 1.867034 & 2.5626 \\
\hline $\mathrm{H}$ & -0.099841 & 2.056904 & 3.6106 \\
\hline H & 0.188125 & 2.304985 & -0.60 \\
\hline
\end{tabular}

\section{TRANS-11 $\left(\mathrm{C}_{9} \mathrm{H}_{12} \mathrm{~N}_{2} \mathrm{O}_{3}\right)$}

$\begin{array}{lrrr}\mathrm{N} & -0.573647 & -1.286026 & -0.327034 \\ \mathrm{C} & -1.535180 & -0.327092 & 0.092083 \\ \mathrm{~N} & -1.040121 & 0.880093 & 0.511592 \\ \mathrm{C} & 0.338023 & 1.275579 & 0.227568 \\ \mathrm{C} & 1.294580 & 0.108360 & 0.540262 \\ \mathrm{C} & 0.785914 & -1.196728 & -0.096194 \\ \mathrm{O} & 1.544519 & -2.131813 & -0.307821 \\ \mathrm{O} & -2.724802 & -0.606069 & 0.080741 \\ \mathrm{C} & -1.095723 & -2.562444 & -0.830706 \\ \mathrm{C} & -1.982443 & 1.935206 & 0.861159 \\ \mathrm{O} & 1.203123 & -0.062744 & 1.960454 \\ \mathrm{C} & 2.733584 & 0.395907 & 0.121073 \\ \mathrm{H} & -2.942543 & 1.475740 & 1.090764 \\ \mathrm{H} & -1.610663 & 2.474791 & 1.738537 \\ \mathrm{H} & -2.113017 & 2.643740 & 0.032522 \\ \mathrm{H} & -1.264565 & -3.265132 & -0.007993 \\ \mathrm{H} & -0.359838 & -2.986888 & -1.512402 \\ \mathrm{H} & -2.040586 & -2.373644 & -1.336066 \\ \mathrm{H} & 2.814040 & 0.567985 & -0.955073\end{array}$




$\begin{array}{lrrr} & & & \\ \mathrm{H} & 1.779253 & -0.810445 & 2.190917 \\ \mathrm{H} & 3.360937 & -0.463819 & 0.371378 \\ \mathrm{H} & 3.099449 & 1.279777 & 0.653502 \\ \mathrm{C} & 0.475533 & 1.797190 & -1.144662 \\ \mathrm{C} & 0.535005 & 2.241615 & -2.265397 \\ \mathrm{H} & 0.594856 & 2.070158 & 0.939415 \\ \mathrm{H} & 0.593359 & 2.621679 & -3.260554\end{array}$


Table 1. B3LYP/6-31G(d) total energies (E, in au) in gas phase.

\begin{tabular}{cc}
\hline Compound & E \\
\hline & \\
\hline Cis-1 & -3182.45012 \\
\hline Trans-1 & -3182.45544 \\
\hline Cis-3 & -766.434393 \\
\hline Trans-3 & -766.426604 \\
\hline Cis-4 & -687.806889 \\
\hline Trans -4 & -687.798888 \\
\hline Cis-5 & -727.117779 \\
\hline Trans-5 & -727.110064 \\
\hline Cis-6 & -843.854306 \\
\hline Trans-6 & -843.846621 \\
\hline Cis-7 & -766.425661 \\
\hline Trans-7 & -766.415177 \\
\hline Cis-8 & -686.570942 \\
\hline Trans-8 & -686.564941 \\
\hline Cis-9 & -840.228070 \\
\hline Trans-9 & -840.221457 \\
\hline Cis-10 & -1160.98264 \\
\hline Trans -10 & -1160.97546 \\
\hline Cis-11 & -685.311916 \\
\hline Trans -11 & -685.306963 \\
\hline
\end{tabular}

Table 2. Spatial distance differences (in Ångström, $\AA$ ) of $H 6$ and $\mathrm{CH}_{3}-9$ between diastereoisomers 1 and 3-11 extracted from DFT calculations
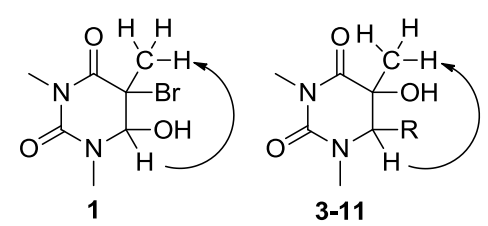

\begin{tabular}{ccccc}
\hline Compound & $\mathbf{R}$ & Cis & Trans & $\Delta$ (trans-cis) \\
\hline $\mathbf{1}$ & - & 3.1 & 3.3 & 0.2 \\
$\mathbf{3}$ & ethyl & 3.1 & 3.2 & 0.1 \\
$\mathbf{4}$ & butyl & 3.1 & 3.2 & 0.1 \\
$\mathbf{5}$ & $i$-propyl & 3.0 & 3.2 & 0.2 \\
$\mathbf{6}$ & cyclohexyl & 3.0 & 3.2 & 0.2 \\
$\mathbf{7}$ & tert-butyl & 3.0 & 3.3 & 0.3 \\
$\mathbf{8}$ & vinyl & 3.0 & 3.2 & 0.2 \\
$\mathbf{9}$ & phenyl & 3.0 & 3.2 & 0.2 \\
$\mathbf{1 0}$ & 2-thienyl & 3.1 & 3.2 & 0.1 \\
$\mathbf{1 1}$ & ethynyl & 3.1 & 3.2 & 0.1 \\
\hline
\end{tabular}




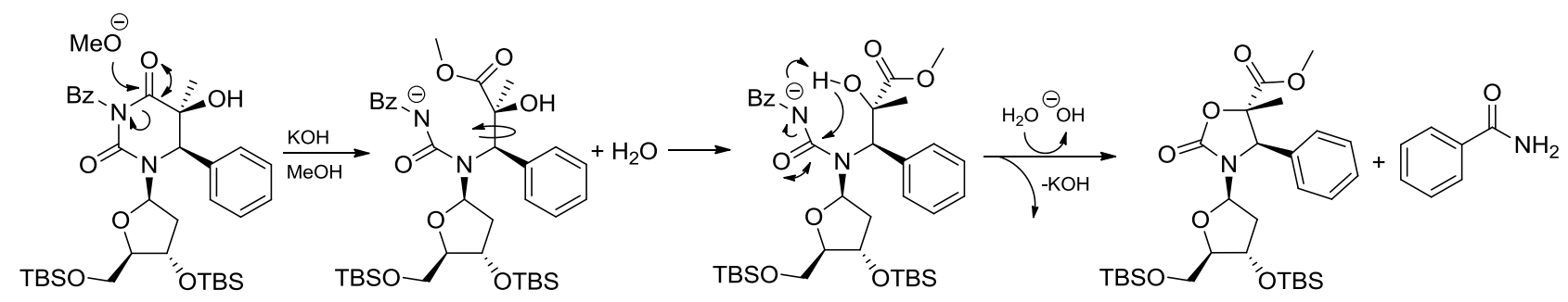

Scheme S1. Putative mechanism for the formation of 2-oxazolidone derivative $\mathbf{3 0}$

(1) Kobzar, K.; Luy, B. J Magn Reson 2007, 186, 131.

(2) Aydin, R.; Günther, H. Magnetic Resonance in Chemistry 1990, 28, 448.

(3) Sheldrick, G. M. Acta Crystallogr A 2008, 64, 112. 